\title{
Metal-Free Synthesis of Indanes by Iodine(III)-Mediated Ring Contraction of 1,2-Dihydronaphthalenes
}

\author{
Fernanda A. Siqueira, ${ }^{a, \#}$ Eloisa E. Ishikawa, ${ }^{a}$ André Fogaça, ${ }^{a}$ Andréa T. Faccio, ${ }^{a}$ \\ Vânia M. T. Carneiro, ${ }^{a}$ Rafael R. S. Soares, ${ }^{a, s}$ Aline Utaka, ${ }^{a}$ Iris R. M. Tébéka, ${ }^{a}$ \\ Marcin Bielawski, ${ }^{a, b}$ Berit Olofsson ${ }^{b}$ and Luiz, F. Silva Jr. $*, a, \not{*}$
}

\author{
${ }^{a}$ Departamento de Química Fundamental, Instituto de Química, Universidade de São Paulo, \\ CP 26077, 05513-970 São Paulo-SP, Brazil \\ ${ }^{b}$ Department of Organic Chemistry, Arrhenius Laboratory, Stockholm University, \\ SE-106 91 Stockholm, Sweden
}

\begin{abstract}
Um protocolo livre de metais foi desenvolvido para sintetizar indanos através da contração de anel de 1,2-di-hidronaftalenos promovida por PhI $(\mathrm{OH}) \mathrm{OTs}$ (HTIB ou reagente de Koser). Este rearranjo oxidativo pode ser realizado em diversos solventes $\left(\mathrm{MeOH}, \mathrm{CH}_{3} \mathrm{CN}\right.$, 2,2,2-trifluoroetanol (TFE), 1,1,1,3,3,3-hexafluoroisopropanol (HFIP), e uma mistura 1:4 de TFE: $\mathrm{CH}_{2} \mathrm{Cl}_{2}$ ) em condições brandas. A contração de anel fornece indanos trans-1,3-dissubstituídos diastereosseletivamente, os quais são difíceis de obter em química orgânica sintética.

A metal-free protocol was developed to synthesize indanes by ring contraction of 1,2-dihydronaphthalenes promoted by $\mathrm{PhI}(\mathrm{OH}) \mathrm{OTs}$ (HTIB or Koser's reagent). This oxidative rearrangement can be performed in several solvents $\left(\mathrm{MeOH}, \mathrm{CH}_{3} \mathrm{CN}, 2\right.$,2,2-trifluoroethanol (TFE), 1,1,1,3,3,3-hexafluoroisopropanol (HFIP), and a 1:4 mixture of TFE: $\mathrm{CH}_{2} \mathrm{Cl}_{2}$ ) under mild conditions. The ring contraction diastereoselectively gives functionalized trans-1,3-disubstituted indanes, which are difficult to obtain in synthetic organic chemistry.
\end{abstract}

Keywords: indanes, hypervalent iodine, ring contraction, 1,2-dihydronaphthalenes, rearrangements

\section{Introduction}

The indane ring system is present in several natural products and in non-natural compounds with remarkable biological activity. ${ }^{1}$ Consequently, efforts have continuously been made to develop new routes to obtain molecules with this unit. ${ }^{2}$ A typical strategy to synthesize a functionalized indane is by selecting an appropriate indanone, which is then elaborated into the target molecule. ${ }^{2,3}$ As tetralones are usually cheaper than indanones, the preparation of indanes starting from a tetralone (or a derivative) through a ring contraction rearrangement could be advantageous. ${ }^{4}$

*e-mail: luizfsjr@iq.usp.br

Present addresses: "Universidade Federal de São Paulo, Campus Diadema, Rua Artur Riedel, 275, 09972-270, Diadema-SP, Brazil

§Instituto Federal de Educação, Ciência e Tecnologia de São Paulo, Rua Pedro Vicente, 625, 01109-010 São Paulo-SP, Brazil

Dedicated with deep respect to Prof. Miuako K. Kuya
In the last years, hypervalent iodine reagents have become an essential tool in synthetic organic chemistry due to the plethora of reactions that can be performed with them in excellent yield and selectivities. ${ }^{5}$ Moreover, hypervalent iodine compounds represent in many cases an alternative to toxic heavy metals. ${ }^{5}$ Although the oxidative rearrangement of alkenes mediated by iodine(III) has been described in some papers, ${ }^{6}$ the ring contraction of 1,2-dihydronaphthalenes was reported for a few substrates using only $p$-Me- $\mathrm{C}_{6} \mathrm{H}_{4}-\mathrm{IF}_{2},{ }^{6}$ which led to fluorinated indanes.

Herein, we describe an efficient metal-free protocol for the synthesis of indanes under mild conditions. In a preliminary communication, we report the ring contraction of 1,2-dihydronaphthalenes (which are obtained from 1-tetralones) mediated by $\mathrm{PhI}(\mathrm{OH}) \mathrm{OTs}$ (HTIB or Koser's reagent) for a few substrates. ${ }^{7}$ In this article, the oxidation of several additional substrates is presented, better defining the reaction scope. Additionally, other reaction conditions were 
discovered using fluoroalcohols as solvent, which highly improved isolated yields. The best condition employed a 4:1 mixture of $\mathrm{CH}_{2} \mathrm{Cl}_{2}$-TFE that led to indanes in very good yield and with high diastereoselectivity.

\section{Results and Discussion}

\section{Ring contractions in methanol}

The required 1,2-dihydronaphthalenes are readily available substrates that can be prepared from 1-tetralones by reduction or Grignard reaction followed by dehydration ${ }^{7,8}$ (see Supplementary Information, SI, for details). This work was initiated studying the oxidation of $\mathbf{1 a}$ with the readily available iodine(III) reagents $\mathrm{HTIB}, \mathrm{PhI}(\mathrm{OAc})_{2}$, and $\mathrm{PhI}\left(\mathrm{OCOCF}_{3}\right)_{2}$ in methanol. Mixtures of several compounds and/or starting material were obtained using $\mathrm{PhI}(\mathrm{OAc})_{2}$ or $\mathrm{PhI}\left(\mathrm{OCOCF}_{3}\right)_{2}$. Albeit the addition product $3 \mathbf{a}$ was isolated as the major component, the desired indane $\mathbf{2 a}$ was isolated using HTIB (Table 1, entry 1). Thus, HTIB was selected for further tests. When the reaction was performed at $-10^{\circ} \mathrm{C}$, the overall isolated yield was lower (2a: $24 \%$, trans-3a: 20\%, cis-3a: $15 \%$ ) than at room temperature. The use of trimethylorthoformate (TMOF) as solvent, instead of $\mathrm{MeOH}$, also decreased the global yield (2a: $14 \%$, trans-3a: 12\%, cis-3a: $2 \%$ ). These two trends are opposite to that observed in analogous thallium(III) promoted oxidation of 1,2-dihydronaphthalenes. ${ }^{9}$ Although indane $2 \mathbf{a}$ was obtained in only $36 \%$, we decided to study the behavior of the methylsubstituted 1,2-dihydronaphthalene $\mathbf{1 b}$, hoping to obtain a higher yield of the ring contraction product. ${ }^{9}$ Indeed, when $\mathbf{1 b}$ was treated with HTIB, the desired trans-indane $\mathbf{2 b}$ was obtained in 55\% yield, together with the addition products $\mathbf{3 b}$ (entry 2). The ring contraction of 1,2-dihydronaphthalene 1c was performed with 3.6 equiv. of HTIB, which delivered indane $2 \mathrm{c}$ in $62 \%$ yield, as a single diastereomer, together with the addition product $\mathbf{3 c}$ in $35 \%$ yield (entry 3 ). With a lower amount of HTIB, the yield of $\mathbf{2 c}$ is smaller. A similar pattern was also observed in $\mathrm{Tl}(\mathrm{III})$ reactions, where an excess of the oxidant increased the yield of the indane.$^{8}$ It is important to note that the diastereoselective synthesis of trans-1,3-disubstituted indanes is a difficult task in synthetic organic chemistry. ${ }^{10}$ Compound $\mathbf{2 c}$ is a synthetic intermediate in the synthesis of $( \pm)$-indatraline, which displays several interesting biological activities. ${ }^{7}$ The presence of donating groups at the aromatic ring may facilitate the rearrangement of 1,2-dihydronaphthalenes by increasing the migratory aptitude of the migrating carbon. ${ }^{8}$ Indeed, the oxidation of alkene 1d, that bears an amide group para to the migrating carbon, with HTIB gave the desired acetal 2d in much higher yield than the corresponding non-substituted substrate 1a
Table 1. Oxidation of 1,2-dihydronaphthalenes with HTIB in $\mathrm{MeOH}$

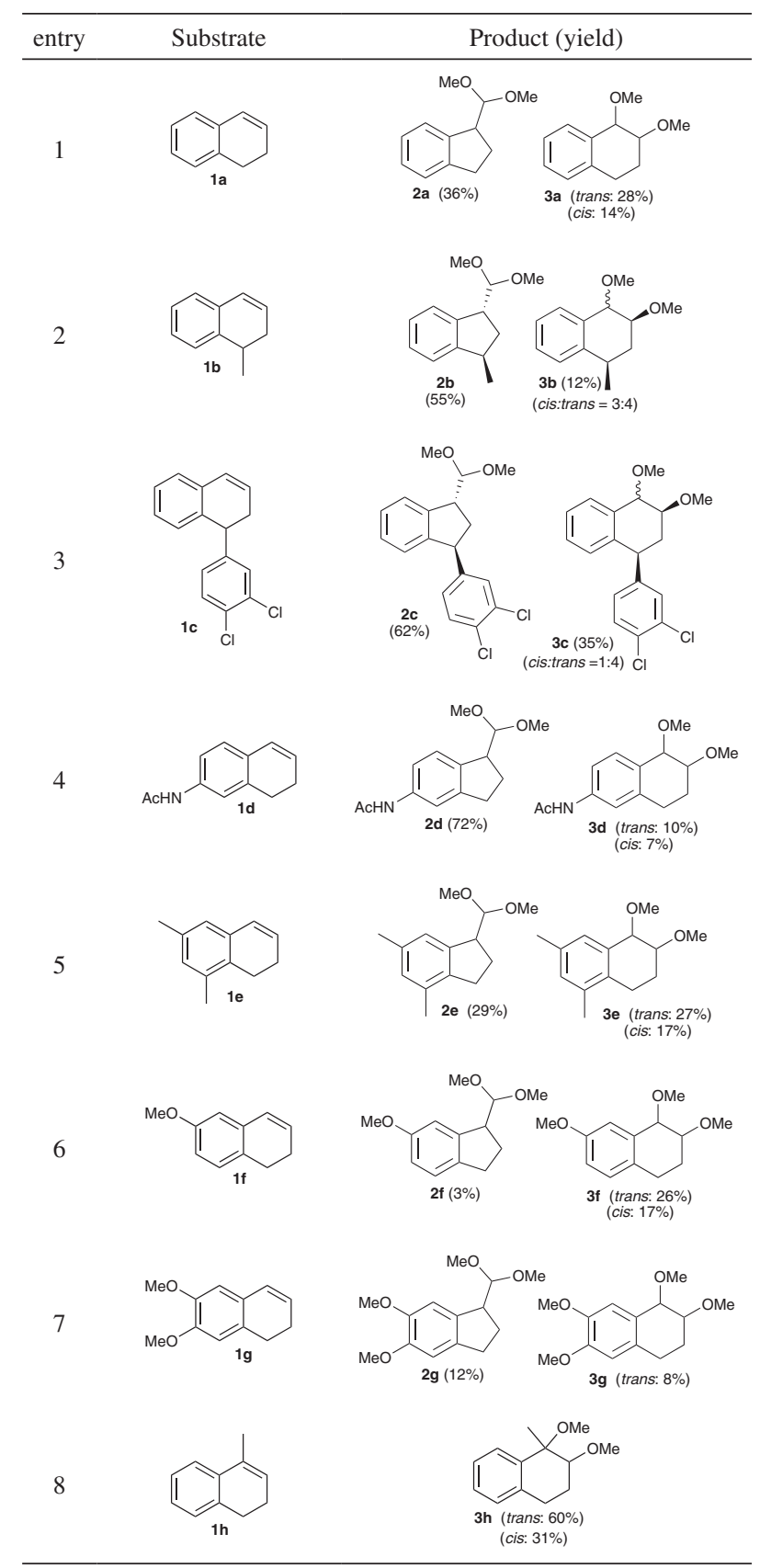

(entry 4). However, the treatment of alkene 1e with HTIB gave indane 2e in comparable yield to that obtained for the substrate 1a ( $c f$. entries 1 and 5). When HTIB was added to a methanol solution of substrates $\mathbf{1 f}-\mathbf{g}$, which bear a methoxy group at the aromatic ring, the mixture immediately became black, leading to indanes $\mathbf{2 f - g}$ in low yield (entries 6 and 7). Low yields in iodine(III)-mediated oxidation of methoxysubstituted substrates has also been observed by others. ${ }^{11,12}$ Considering our experience in the oxidations of alkenes mediated by Tl(III), ${ }^{9}$ we expected that the trisubstituted 1,2-dihydronaphthalene $\mathbf{1 h}$ would have a different behavior 
toward HTIB from that of the disubstituted alkene 1a. Indeed, when $\mathbf{l h}$ was treated with HTIB in $\mathrm{MeOH}$ only the addition product $\mathbf{3 h}$ was isolated (entry 8 ). It is important to note that the acetal moiety in indanes like 2a-g can be easily transformed without epimerization into the corresponding aldehyde. ${ }^{2}$

\section{Ring contractions in acetonitrile}

The conditions used by Kirschning and co-workers ${ }^{6}$ in the oxidation of carbohydrates were also applied in the oxidation of 1,2-dihydronaphthalenes. Naphthalene (4a) was isolated in 30\% yield when 1a was treated with HTIB in $\mathrm{CH}_{3} \mathrm{CN}$ (Table 2, entry 1). NMR analysis of the crude product indicates the presence of indane $\mathbf{5 a}$ as a minor component, which decomposed during the purification step. ${ }^{13}$ Similarly, 4 a was obtained in $48 \%$ yield when the reaction was performed in $\mathrm{CH}_{2} \mathrm{Cl}_{2}$, as solvent. However, when $\mathbf{1 h}$ was treated with HTIB in $\mathrm{CH}_{3} \mathrm{CN}$ indane $\mathbf{5} \mathbf{h}$ was isolated in $51 \%$ yield (entry 2 ), which should be compared to exclusive formation of addition products in $\mathrm{MeOH}$ reactions (Table 1, entry 8). Ring contractions of epoxides can also be performed by treatment with Brønsted or Lewis acids. ${ }^{4}$ However, compound $\mathbf{5 h}$ can not be prepared in this manner, as no ring contraction product was obtained from the epoxide prepared from $1 \mathbf{h} .{ }^{14-17}$ The oxidative rearrangement of other 4-alkyl-1,2-dihydronaphthalenes was also investigated. The reaction of alkenes $1 \mathbf{i}$ and $\mathbf{1 g}$, which bear a methoxy group in the aromatic ring, with $\mathrm{HTIB}$ in $\mathrm{CH}_{3} \mathrm{CN}$ furnished indanes $5 \mathbf{i}$ and $\mathbf{5 g}$, respectively, in low yield (Table 2, entries 3 and 4), similarly to the disubstituted alkenes (Table 1, entries 6-7). Trisubstituted alkenes $\mathbf{1 k}-\mathbf{m}$ were transformed into indanes $\mathbf{5 k}-\mathbf{m}^{13}$ in good yield (entries 5-7). Thus, the ring contraction is not precluded by the presence of bulky alkyl groups. The behavior of alkene $\mathbf{1 n}$ is slightly different to that observed for other substrates. The reaction of $\mathbf{1 n}$ with $\mathrm{HTIB}$ in $\mathrm{CH}_{3} \mathrm{CN}$ led mainly to indane $\mathbf{5 n}$ and ketone $\mathbf{6 n}^{18}$ in 26 and $23 \%$ yield, respectively. The tetralone $\mathbf{6} \mathbf{n}$ is formed by migration of the phenyl group. ${ }^{6}$ The reaction of $\mathbf{1} \mathbf{n}$ with HTIB led to a nearly 1:1 mixture of the rearrangement products $5 \mathbf{n}$ and $\mathbf{6 n}$, because the aromatic rings have similar migratory aptitude. In theory, if the migratory aptitude of the aromatic rings was different, the ratio of the rearrangement products could be modified. Indeed, when 1o, which has two $\mathrm{Cl}$ atoms in one of the rings, was treated with HTIB, trans-indane $\mathbf{5 0}$ was isolated and the product of migration of the $\mathrm{C}_{6} \mathrm{H}_{3} \mathrm{Cl}_{2}$ group was not formed, because of the low migratory aptitude of $\mathrm{C}_{6} \mathrm{H}_{3} \mathrm{Cl}_{2}$. However, a small amount of the tetralone 7o, which is formed by migration of hydride, ${ }^{13,16}$ was isolated (entry 9). Finally, we investigated the ring contraction in a seven-membered ring substrate. When alkene $\mathbf{1 p}$ was
Table 2. Oxidation of 1,2-dihydronaphthalenes with $\mathrm{HTIB}$ in $\mathrm{CH}_{3} \mathrm{CN}$

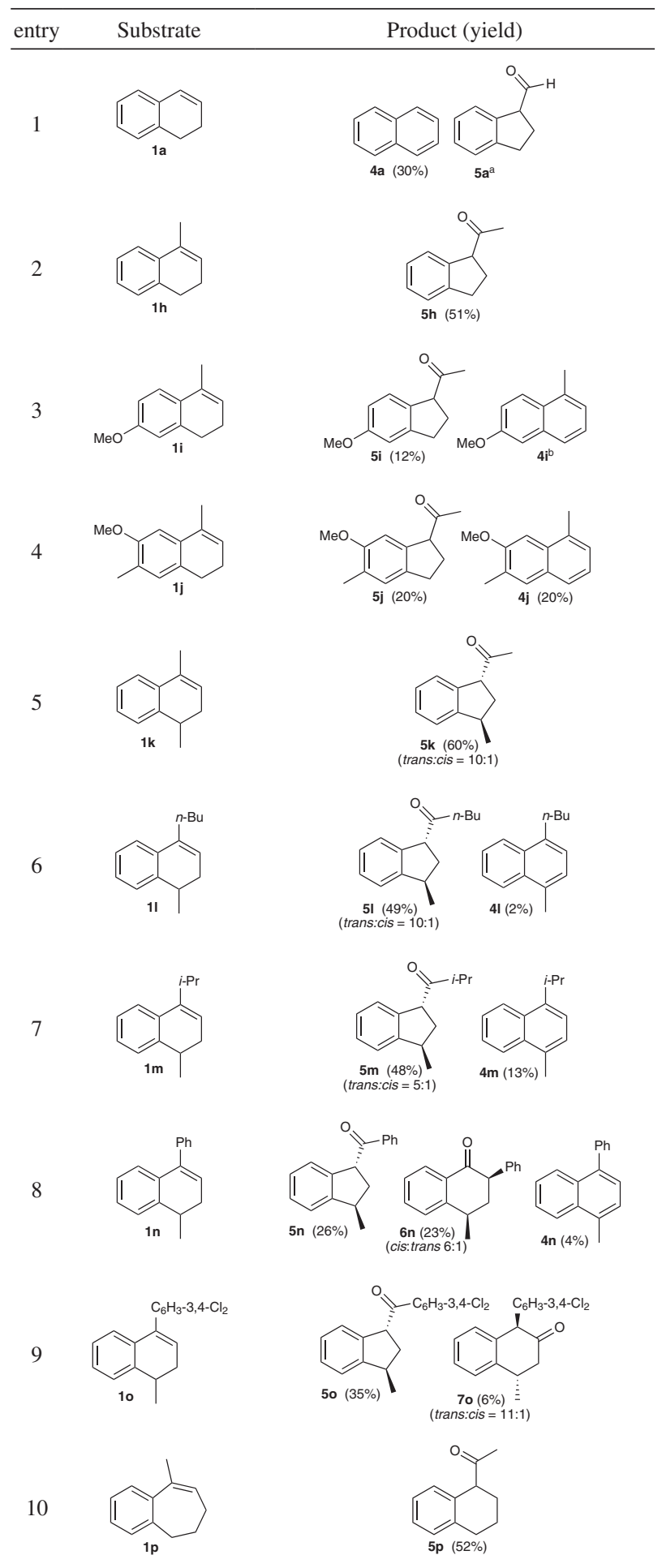

${ }^{a}$ Yield not determined; b together with 1i, ca. 20\%.

treated with $\mathrm{HTIB}$ in $\mathrm{CH}_{3} \mathrm{CN}$, the substituted tetralin $\mathbf{5 p}$ was obtained in good yield (entry 10). The ring contractions in $\mathrm{CH}_{3} \mathrm{CN}$ were performed under inert atmosphere and in the presence of molecular sieves. When these conditions were 
not followed, lower yields were observed. The preparation of indanes analogues to $\mathbf{5}$ from 1,2-dihydronaphthalenes has been reported in a two-step protocol using NBS/water followed by reaction with $\mathrm{Et}_{2} \mathrm{Zn}$, which requires anhydrous conditions. ${ }^{17}$

\section{Ring contractions in fluorinated solvents}

After investigating the oxidation of 1,2-dihydronaphthalenes with HTIB in methanol and in acetonitrile, we focused on the more polar solvents 2,2,2-trifluoroethanol (TFE) and 1,1,1,3,3,3-hexafluoroisopropanol (HFIP) because we envisioned that the formation of by products could be decreased performing the reaction in these high polar low nucleophilic solvents. Since the first report by Kita et al..${ }^{19}$ the fluoroalcohols TFE ${ }^{20}$ and HFIP ${ }^{21}$ have been used as solvent in several reactions with hypervalent iodine compounds. However, TFE and HFIP have never been used in the oxidative rearrangement of alkenes. ${ }^{5,6}$

For the alkene 1a, the yield of the desired product jumped from $36 \%$ ( $c f$. entry 1, Table 1 ) to more than the double ( $73 \%$, Table 3, entry 1). The reaction of $\mathbf{1 b}$ with HTIB in TFE led to indane $\mathbf{8 b}$ in higher yield than in $\mathrm{MeOH}$ (55\% vs. 70\%), although the diastereoselectivity is lower (entry 2 of Tables 1 and 3 , respectively). The ring contraction of $1 \mathbf{q}$ in TFE led to $\mathbf{8 q}$ in 65\% yield, as a 10:1 mixture of trans:cis diastereomers, respectively. Considering our previous work on the synthesis of 3-phenyl-1-indanamines, ${ }^{7}$ the indane $\mathbf{8 q}$ could be used as an intermediate in the synthesis of ( \pm )-irindalone. ${ }^{22}$ Moreover, this new method to obtain fluorinated acetals, which have different applications, ${ }^{23}$ is more efficient than the previous described. ${ }^{24-26}$ The oxidation of trisubstituted alkenes $\mathbf{1 h}$ and $\mathbf{1 p}$ with HTIB in TFE gave indanes $\mathbf{5 h}$ and $\mathbf{5 p}$, respectively, in higher yield than using acetonitrile ( $c f$. Table 2, entries 2 and 10 with entries 4 and 7 of Table 3). On the other hand, $\mathbf{1 k}$ led to $\mathbf{5 k}$ in lower yield and diastereoselectivity than in acetonitrile (entry 5 of Tables 2 and 3).

Although the HTIB-mediated oxidation of 1,2-dihydronaphthalenes in TFE led to the rearrangement products in higher yields than in other solvents, the diastereoselectivity is lower. Thus, several conditions were tested trying to optimize the diastereoselectivity, without decreasing the isolated yields. Eventually, this goal was achieved by performing the reaction in a $4: 1$ mixture of $\mathrm{CH}_{2} \mathrm{Cl}_{2}$ :TFE as solvent. Although $\mathrm{CH}_{2} \mathrm{Cl}_{2}$ is the major component of the mixture, TFE must have a crucial role because the reaction of $1 \mathbf{a}$ with HTIB in pure $\mathrm{CH}_{2} \mathrm{Cl}_{2}$ gave naphthalene ( $c f$. entry 1 , Table 2 ). The indane $\mathbf{8 a}$ was obtained from 1a in a yield comparable to the reaction in only TFE ( $73 \%$ vs. $67 \%$ yield, entry 1 , Table 3 ). The alkene 1q gave the indane $\mathbf{8 q}$ in $69 \%$ yield, as a trans:cis
Table 3. Reaction of 1,2-dihydronaphthalenes with HTIB in fluoroalcohols ${ }^{\mathrm{a}}$

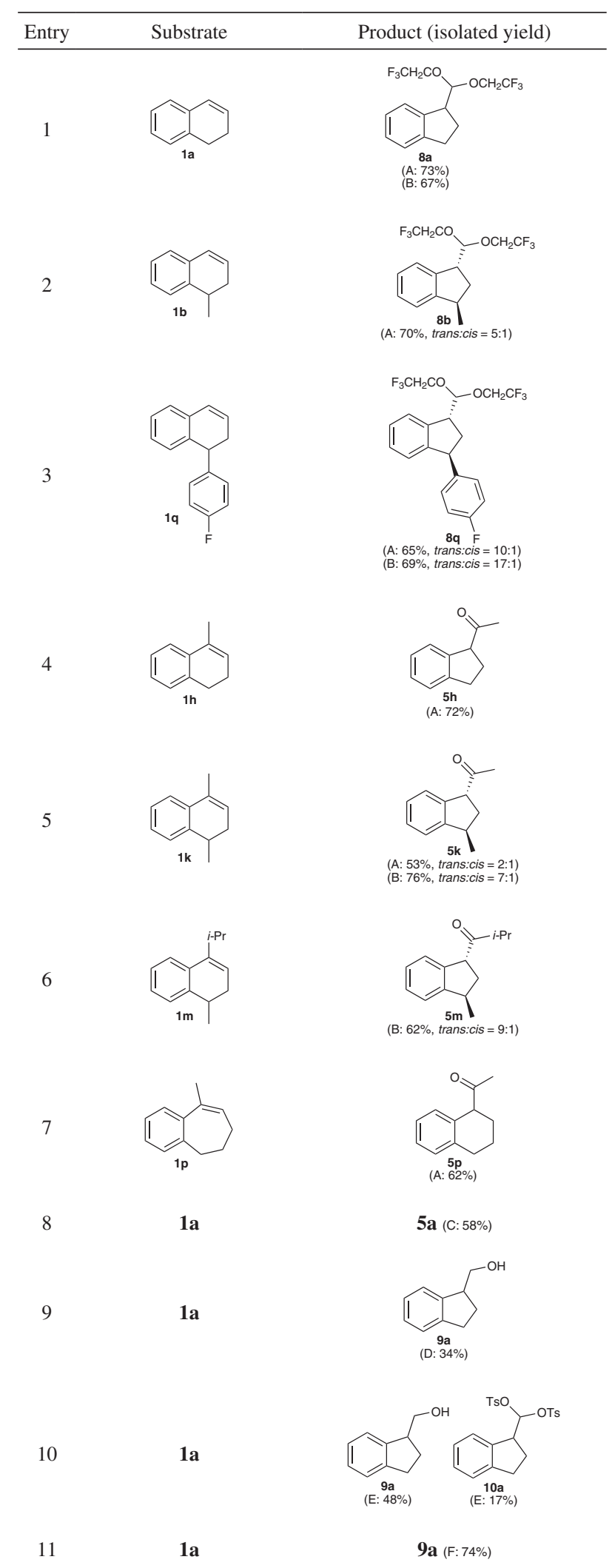

${ }^{a}$ A: TFE; B: 4:1 mixture of $\mathrm{CH}_{2} \mathrm{Cl}_{2}$ /TFE; C: HFIP; D: i) HFIP, ii) $\mathrm{NaBH}_{4}$; E: i) $\mathrm{CH}_{2} \mathrm{Cl}_{2}: \mathrm{HFIP}(4: 1)$, ii) $\mathrm{NaBH}_{4}$; F: i) 22 equiv. $\mathrm{H}_{2} \mathrm{O}, \mathrm{CH}_{2} \mathrm{Cl}_{2}: \mathrm{HFIP}$ (4:1), ii) $\mathrm{NaBH}_{4}$. 


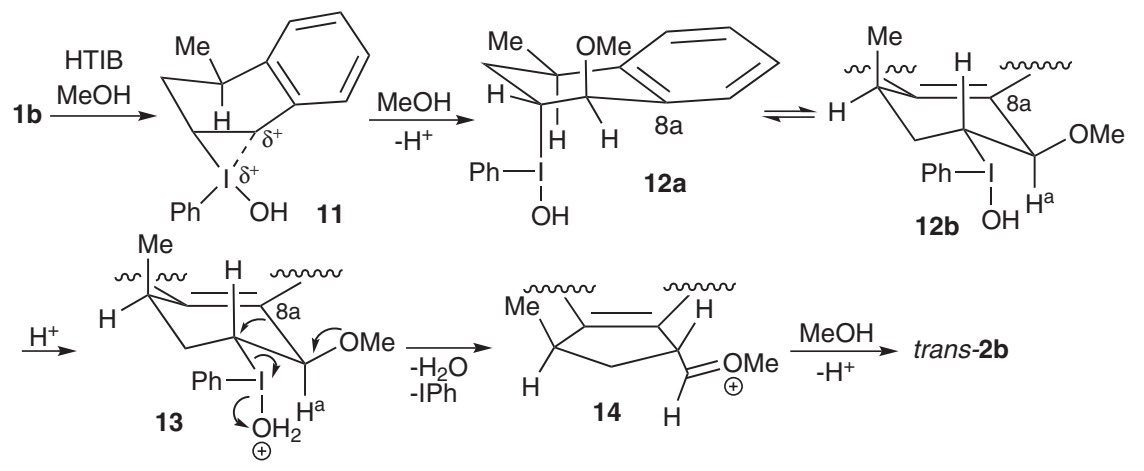

Scheme 1. Mechanism for the ring contraction of $\mathbf{1 b}$ in $\mathrm{MeOH}$.

ratio of 17:1, i.e., in better yield and selectivity than using only TFE (entry 3, Table 3). The reaction in $\mathrm{CH}_{2} \mathrm{Cl}_{2} / \mathrm{TFE}$ is also appropriate for trisubstituted alkenes. Ketones $\mathbf{5 k}$ and $\mathbf{5 m}$ were obtained in higher yield than in acetonitrile or in TFE. Furthermore, the diastereoselectivity is higher than in TFE and comparable to the reaction in acetonitrile (cf. entries 5 and 7 of Table 2 and entries 5 and 6 of Table 3 ). In summary, treatment of 1,2-dihydronaphthalenes with HTIB in TFE or in $\mathrm{CH}_{2} \mathrm{Cl}_{2} / \mathrm{TFE}$ gave the desired indanes in higher yields than using $\mathrm{MeOH}$ or $\mathrm{CH}_{3} \mathrm{CN}$ for either di- or trisubstituted double bonds.

Considering the very good results with TFE, the obvious extension would be the study of the reaction in the even more polar solvent HFIP. The oxidation of alkene 1a in HFIP was very fast and led to indane 5a. The yield of the ring contraction product was, however, lower than in TFE (cf. entries 1 and 8, Table 3). In the presence of the bulky and low nucleophilic solvent HFIP an aldehyde (5a) is isolated instead of acetals, as in $\mathrm{MeOH}$ or in TFE (2a and 8a). Aldehyde 5a is not very convenient for manipulation and storage because it decomposes. We thus investigated if $\mathbf{5 a}$ could be reduced in situ, giving a stable alcohol. The reaction of 1a with HTIB in HFIP followed by addition of $\mathrm{NaBH}_{4}$ gave the desired alcohol 9a in only $34 \%$ yield (entry 9). Changing the solvent to a mixture of $\mathrm{CH}_{2} \mathrm{Cl}_{2}$ :HFIP (4:1), the alcohol 9a was isolated in better yield, however together with the gem ditosylate $\mathbf{1 0 a}^{6}$ in $17 \%$ yield (entry 10 ). We envisioned that the addition of $\mathrm{H}_{2} \mathrm{O}$ could favor the formation of $\mathbf{9 a}$, avoiding the undesired product $\mathbf{1 0 a}$. Indeed, a smooth ring contraction/reduction was observed when 1a was treated with HTIB in the presence of $\mathrm{H}_{2} \mathrm{O}$ in $\mathrm{CH}_{2} \mathrm{Cl}_{2} / \mathrm{HFIP}$ (4:1) as solvent, followed by addition of $\mathrm{NaBH}_{4}$, giving 9a in 74\% isolated yield (entry 11).

\section{Mechanism discussion}

The exclusive formation of trans-1,3-disubstituted indanes in the ring contractions in methanol can be explained by the mechanism detailed below. The electrophilic anti-addition of HTIB to the double bond would lead to $\mathbf{1 2 a}$, through the cyclic organoiodine intermediate 11. The approach of the electrophile occurs opposite to the remote methyl group, ${ }^{27-29}$ explaining the stereoselectivity of this ring contraction, as well as of the other reactions discussed below. The adduct $\mathbf{1 2 a}$ would equilibrate to its more stable conformational isomer $\mathbf{1 2 b}$, on which the required anti-periplanarity for the rearrangement is achieved. Migration of the aryl group (carbon 8a) on $\mathbf{1 3}$ would displace $\mathrm{PhI}$ giving the oxonium $\mathbf{1 4}$, which would furnish the trans-indane $\mathbf{2 b}$ after addition of $\mathrm{MeOH}$ (Scheme 1). The diastereoselective formation of the trans products in ring contractions in TFE or in $\mathrm{CH}_{2} \mathrm{Cl}_{2}$ /TFE can be explained by similar mechanisms. However, considering the anhydrous conditions of the ring contraction in $\mathrm{CH}_{3} \mathrm{CN}$, the mechanism is probably different, as shown in Scheme 2 for $1 \mathbf{n}$. The stereoselective electrophilic addition of HTIB to the alkene $1 \mathbf{n}$ would give the bis-benzylic carbocation 15. The hydroxyl group would attack the $\mathrm{C} 1$ position of $\mathbf{1 5}$, giving the four-membered ring intermediate $16,{ }^{6}$ which would ring open to form 17 . The ring contraction would take place on its conformer (18) giving trans-5n (path a, Scheme 2). The solvent may have some influence in the stereoselectivity of the electrophilic addition of the iodine(III), explaining the formation of

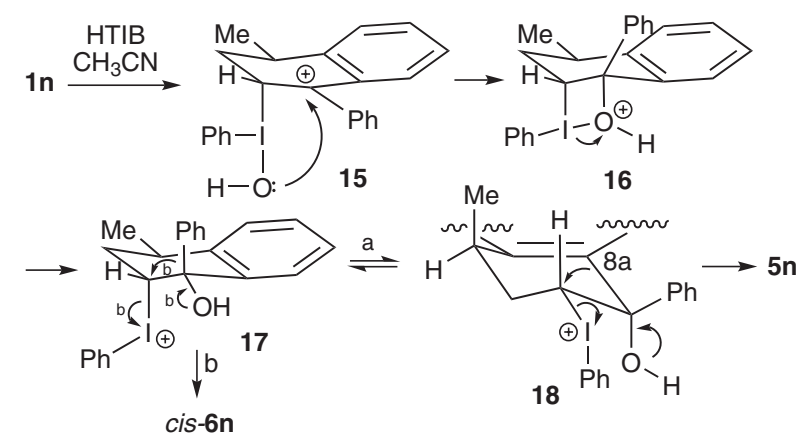

Scheme 2. Rearrangements of $\mathbf{1 n}$ in $\mathrm{CH}_{3} \mathrm{CN}$. 
cis-1,3-disubstituted indanes. Alternatively, the cis indanes can be formed by epimerization of the ketone moiety of the corresponding trans isomers. Starting from trisubstituted double bonds, the ring contraction lead to ketones which are always obtained as a free carbonyl. Aldehydes are formed from disubstituted alkenes. In the presence of a nucleophilic solvent, such as $\mathrm{MeOH}$ or TFE, acetals were isolated. On the other hand, free aldehydes were obtained in $\mathrm{CH}_{3} \mathrm{CN}$ or in HFIP.

The formation of the cis-2,4-disubstituted-1-tetralone 6n can be explained by the mechanism shown in path $b$ of Scheme 2. The Ph group would migrate on intermediate 17, with the exit of PhI, leading to cis-6n. trans-6n can be formed either by isomerization of $c i s$-isomer or the addition of I(III) to 1 n could take place by the other face. In acetonitrile oxidations, small amounts of naphthalenes were isolated in some reactions, which are formed by addition followed by elimination. ${ }^{13}$

A plausible mechanism to explain the formation of the products of addition of $\mathrm{MeOH}$ is shown in Scheme 3, using substrate 1a as example. ${ }^{6,8}$ The methoxy group of 19 would intramolecularly displace PhI, giving the oxonium 20. Methanol would attack the $\mathrm{C} 1$ benzylic position of 20, furnishing trans-3a (path a). Alternatively, the intermolecular displacement of $\mathrm{PhI}$ by $\mathrm{MeOH}$ in the intermediate $\mathbf{1 9}$ would lead to cis-3a (path b). The preferential formation of the trans isomers (Table 1) indicates that the intramolecular process is favored. The formation of cis- and trans-isomers has also been observed in the reaction of indene with iodosobenzene derivatives in methanol. ${ }^{30}$ However, the oxidation of cyclohexenes with iodine(III) led to rearrangement products, ${ }^{6}$ cis-isomers ${ }^{6,31-33}$ or trans-isomers, ${ }^{31,34}$ depending mainly on the reaction conditions.
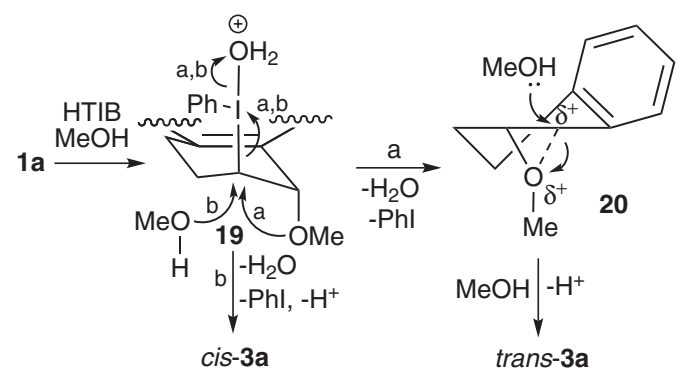

Scheme 3. Mechanism for the formation of addition products 3a.

As described above, the solvent has a crucial role in the oxidation of 1,2-dihydronaphthalenes with HTIB. In methanol, ring contraction is favored toward the addition of solvent for disubstituted double bonds. However, for trisubstituted substrates, the nucleophilic attack of $\mathrm{MeOH}$ is faster, probably because the required conformations for the rearrangements are disfavored with an additional methyl group (12b and 13 with Me instead $\mathrm{H}^{\mathrm{a}}$ in Scheme 1). In anhydrous acetonitrile, there is no good nucleophile and ring contraction of trisubstituted alkenes occurs through the formation of a tertiary benzylic carbocation (like 15). For disubstituted double bonds, the ring contraction would occur through a less favored secondary benzylic carbocation and, thus, the formation of naphthalenes predominates. In TFE or in $\mathrm{CH}_{2} \mathrm{Cl}_{2} / \mathrm{TFE}$, ring contraction was observed for either di- or trisubstituted 1,2-dihydronaphthalenes. The mechanism described for $\mathrm{MeOH}$ is the major pathway, as acetals are isolated for disubstituted alkenes. Ring contraction also takes place with trisubstituted substrates, because a less nucleophilic species is present, making the formation of addition products more difficult.

\section{Conclusions}

A one-step, fast, mild and metal-free protocol was developed for the synthesis of indanes through ring contraction of readily available 1,2-dihydronaphthalenes mediated by HTIB. This oxidative rearrangement is diastereoselective giving 1,3-trans-disubstituted indanes preferentially or exclusively. The developed methodology facilitates the access to this structural motif, which is difficult to construct. Moreover, indanes bearing different functional groups can be easily obtained by changing the reaction conditions. In summary, the protocol herein presented will be useful in synthetic organic chemistry and in medicinal chemistry to access functionalized indanes in an expeditious manner. The protocol represents a green alternative to the analogous reaction using toxic thallium(III) salts. ${ }^{8,9,13,35,36}$

\section{Experimental}

\section{General procedure}

Synthesis of 4-(4-fluorophenyl)-3,4-dihydronaphthalen1(2H)-one

To a dry round bottom flask under nitrogen atmosphere, $\mathrm{AlCl}_{3}(7.8 \mathrm{~g}, 59 \mathrm{mmol})$ was added followed by the addition of fluorobenzene $(10.8 \mathrm{~mL}, 11.1 \mathrm{~g}, 115 \mathrm{mmol})$. After cooling the flask to $0{ }^{\circ} \mathrm{C}, 1$-naftol $(3.0 \mathrm{~g}, 20.8 \mathrm{mmol})$ was added portion-wise under strong stirring (cake forms). After the addition, the flask was charged with a condenser and stirred at $75^{\circ} \mathrm{C}$ for $1.5 \mathrm{~h}$. The reaction was again cooled to $0{ }^{\circ} \mathrm{C}$ and quenched by adding ice through the condenser (strongly exothermic), until no gas evolution could be observed. The reaction mixture was extracted with $\mathrm{CH}_{2} \mathrm{Cl}_{2}$ $(3 \times 25 \mathrm{~mL})$, the organics washed with $1 \mathrm{~mol} \mathrm{~L}^{-1} \mathrm{NaOH}$ 
$(2 \times 20 \mathrm{~mL})$ and brine, dried with $\mathrm{Na}_{2} \mathrm{SO}_{4}$, filtered and concentrated to give a thick brown oil $(5.48 \mathrm{~g})$. The crude oil was purified by column chromatography $\left(10 \% \mathrm{Et}_{2} \mathrm{O}\right.$ in hexane), where the $o$-isomer elutes first followed by the $m$ - and $p$-isomers. As the $m$ - and $p$-isomers have the same $R_{\mathrm{f}}$ value, the mixed fractions were checked by GC to collect fractions with pure $p$-product 4-(4-fluorophenyl)3,4-dihydronaphthalen-1(2H)-one ${ }^{37}$ (1.14 g, $4.75 \mathrm{mmol}$, $23 \%)$.

Synthesis of 1-(4-fluorophenyl)-1,2-dihydronaphthalene (1c)

4-(4-fluorophenyl)-3,4-dihydronaphthalen-1(2H)-one $(806 \mu \mathrm{L}, 3.36 \mathrm{mmol})$ was added to a round bottom flask, diluted with $\mathrm{MeOH}(25 \mathrm{~mL})$ followed by cooling to $0{ }^{\circ} \mathrm{C}$ and addition of $\mathrm{NaBH}_{4}(140 \mathrm{mg}, 3.68 \mathrm{mmol})$. The reaction was quenched with $\mathrm{H}_{2} \mathrm{O}$ after $1 \mathrm{~h}$ and adjusted to $\mathrm{pH} 5$ with $10 \% \mathrm{HCl}$. After evaporation of the $\mathrm{MeOH}$, the aqueous phase was extracted with EtOAc $(3 \times 15 \mathrm{~mL})$, followed by the washing of the organics with brine, dried with $\mathrm{Na}_{2} \mathrm{SO}_{4}$, filtered and concentrated to give a crude yellow oil of 4-(4-fluorophenyl)-1,2,3,4-tetrahydronaphthalen1-ol (928 mg), which was used in the next step without any further purification. The crude tetralol (928 $\mathrm{mg}$ ) was added to a dry round bottom flask, followed by dry toluene $(20 \mathrm{~mL})$ and a few crystals of $p$-TsOH (cat.). The flask was equipped with a dean-stark trap and refluxed until no alcohol remained according to TLC $(c a .1 .5 \mathrm{~h})$. The reaction was quenched with a saturated $\mathrm{NaHCO}_{3}$ solution and diluted with EtOAc. The organic phase was washed with saturated $\mathrm{NaHCO}_{3}(2 \times 15 \mathrm{~mL})$, brine $(2 \times 15 \mathrm{~mL})$, dried with $\mathrm{Na}_{2} \mathrm{SO}_{4}$, filtered and concentrated to give a crude brown oil (761 mg). It was purified by column chromatography (hexane) to afford 1c as colorless oil (728 mg, $3.25 \mathrm{mmol}$, $97 \%$ over 2 steps); ${ }^{1} \mathrm{H}$ NMR (400 MHz, $\left.\mathrm{CDCl}_{3}\right) \delta 2.56$ (dddd, $1 \mathrm{H}, J$ 13.8, 9.7, 4.2 and $2.0 \mathrm{~Hz}$ ), 2.67 (dddd, $1 \mathrm{H}$, $J 12.1,7.4,4.6$ and $1.8 \mathrm{~Hz}), 4.12(\mathrm{dd}, 1 \mathrm{H}, J 9.3$ and $7.7 \mathrm{~Hz}$ ), $5.99(\mathrm{dt}, 1 \mathrm{H}, J 9.6$ and $4.4 \mathrm{~Hz}), 6.55(\mathrm{dt}, 1 \mathrm{H}, J 9.6$ and $1.5 \mathrm{~Hz}), 6.81(\mathrm{~d}, 1 \mathrm{H}, J 7.7 \mathrm{~Hz}), 7.03-6.95(\mathrm{~m}, 2 \mathrm{H}), 7.14-7.07$ (m, 2H), 7.22-7.14 (m, 3H); ${ }^{13} \mathrm{C}$ NMR (75 MHz, $\mathrm{CDCl}_{3}$ ) $\delta 32.2,43.2,115.3(\mathrm{~d}, J 21 \mathrm{~Hz}), 126.4,127.1,127.1,127.5$, 127.9, 128.2, 129.9 (d, $J 8 \mathrm{~Hz}), 134.2,137.8,140.3$ (d, $J 3 \mathrm{~Hz}), 161.7(\mathrm{~d}, J 245 \mathrm{~Hz})$; HRMS $\left(\mathrm{m} / \mathrm{z}\right.$ ) calcd. for $\mathrm{C}_{16} \mathrm{H}_{13} \mathrm{~F}$ $247.0893[\mathrm{M}+\mathrm{Na}]^{+}$, found 247.0901 .

\section{Reaction of 1,2-dihydro-6-methoxynaphthalene (1f) with $\mathrm{HTIB}$ in $\mathrm{MeOH}$}

To a solution of $\mathbf{1 f}(0.328 \mathrm{~g}, 2.05 \mathrm{mmol})$ in $\mathrm{MeOH}$ $(8 \mathrm{~mL})$ was added HTIB $(0.941 \mathrm{~g}, 2.40 \mathrm{mmol})$ at $0{ }^{\circ} \mathrm{C}$. Immediately after addition of HTIB the reaction became dark. The mixture was stirred at room temperature for $1 \mathrm{~h}$. The reaction was extracted with EtOAc, washed with $\mathrm{H}_{2} \mathrm{O}$, with brine, and dried over anhydrous $\mathrm{MgSO}_{4}$. The solvent was removed under reduced pressure. The crude product was purified by column $(0-25 \%$ EtOAc in hexane), affording $2 \mathbf{f}^{9}(0.0137 \mathrm{~g}, 0.0616 \mathrm{mmol}, 3 \%)$, as colorless oil, trans-3f ${ }^{9}(0.117 \mathrm{~g}, 0.526 \mathrm{mmol}, 26 \%)$ and cis-3f $(0.0779 \mathrm{~g}, 0.350 \mathrm{mmol}, 17 \%)$, both as yellow oils. cis-1,2,3,4-Tetrahydro-1,2,7-trimethoxynaphthalene (3f): colorless oil; IR $v_{\max } / \mathrm{cm}^{-1}$ (film) 1101, 1249, 1499, 2834, 2933; ${ }^{1} \mathrm{H}$ NMR (500 MHz, $\left.\mathrm{CDCl}_{3}\right) \delta$ 1.91-1.94 (m, 1H), 2.16-2.23 (m, 1H), 2.67-2.74 (m, 1H), 2.92-2.96 (m, 1H), $3.47(\mathrm{~s}, 3 \mathrm{H}), 3.50(\mathrm{~m}, 3 \mathrm{H}),, 3.61-3.69(\mathrm{~m}, 1 \mathrm{H}), 3.79(\mathrm{~s}, 3 \mathrm{H})$, $4.30(\mathrm{~d}, 1 \mathrm{H}, J 3.1 \mathrm{~Hz}), 6.80(\mathrm{dd}, 1 \mathrm{H}, J 8.3,2.6 \mathrm{~Hz}), 6.86(\mathrm{~d}$, $1 \mathrm{H}, J 2.6 \mathrm{~Hz}), 7.04$ (d, 1H, J 8.3 Hz); ${ }^{13} \mathrm{C}$ NMR $(75 \mathrm{MHz}$, $\left.\mathrm{CDCl}_{3}\right) \delta 22.7,26.1,55.3,56.5,57.4,77.9,78.2,114.3$, 114.4, 128.5, 129.7, 135.7, 157.5; LRMS ( $/ / z, \%) 222\left(\mathrm{M}^{+*}\right.$, 17), 191 (7), 190 (52); 189 (9), 164 (100); HRMS (m/z) calcd. for $\mathrm{C}_{13} \mathrm{H}_{18} \mathrm{O}_{3}[\mathrm{M}+\mathrm{Na}]^{+} 245.1148$, found 245.1141 .

Reaction of 1,2-dihydro-6,7-dimethoxynaphthalene (1g) with $\mathrm{HTIB}$ in $\mathrm{MeOH}$

As 1f, but using 1 g (0.0744 g, $0.391 \mathrm{mmol})$, HTIB $(0.153 \mathrm{~g}, 0.391 \mathrm{mmol})$, and $\mathrm{MeOH}(2.0 \mathrm{~mL})$. The reaction was stirred for $1 \mathrm{~h}$ at $0{ }^{\circ} \mathrm{C}$. The crude product was purified by column (0-30\% EtOAc in hexane), affording $2 \mathbf{g}^{8}(0.0116 \mathrm{~g}$, $0.0460 \mathrm{mmol}, 12 \%)$ and trans-3g $(0.0080 \mathrm{~g}, 0.032 \mathrm{mmol}$, $8 \%$, both as a colorless oil. trans-1,2,3,4-Tetrahydro1,2,6,7-tetramethoxynaphthalene (3g): colorless oil; IR $v_{\text {max }} / \mathrm{cm}^{-1}$ (film) 1121, 1258, 1515, 2830, 2934; ${ }^{1} \mathrm{H}$ NMR (300 MHz, $\mathrm{CDCl}_{3}$ ) $\delta$ 1.88-1.97 (m, 1H), 2.05-2.15 (m, $1 \mathrm{H}), 2.62-2.82(\mathrm{~m}, 2 \mathrm{H}), 3.44(\mathrm{~s}, 3 \mathrm{H}), 3.51(\mathrm{~s}, 3 \mathrm{H}), 3.71$ (ddd, $1 \mathrm{H}, J 7.2,4.8,2.7 \mathrm{~Hz}), 3.84(\mathrm{~s}, 3 \mathrm{H}), 3.87(3 \mathrm{H}, \mathrm{s})$, 4.21 (d, 1H, J 4.8 Hz), $6.58(1 \mathrm{H}), 6.83(\mathrm{~s}, 1 \mathrm{H}) ;{ }^{13} \mathrm{C}$ NMR $\left(75 \mathrm{MHz}, \mathrm{CDCl}_{3}\right) \delta 23.4,25.1,55.9,56.0,56.7,57.2,77.2$, $79.5,111.1,112.4,126.5,129.3,147.5,148.7 ; \operatorname{HRMS}(\mathrm{m} / \mathrm{z})$ calcd. for $\mathrm{C}_{14} \mathrm{H}_{20} \mathrm{O}_{4}[\mathrm{M}+\mathrm{Na}]^{+} 275.1254$, found 275.1252 .

Synthesis of 1-(dimethoxymethyl)-5-acetamido-indane (2d)

To a stirred mixture of $\mathbf{1 d}(0.254 \mathrm{~g}, 1.36 \mathrm{mmol})$ and $\mathrm{MeOH}(27 \mathrm{~mL})$, was added HTIB $(0.590 \mathrm{~g}, 1.50 \mathrm{mmol})$ at once at $0{ }^{\circ} \mathrm{C}$. After $35 \mathrm{~min}$ the reaction was quenched with saturated solution of $\mathrm{NaHCO}_{3}$. The aqueous phase was extracted with EtOAc $(3 \times 10 \mathrm{~mL})$, washed with brine $(2 \times 10 \mathrm{~mL})$ and dried over anhydrous $\mathrm{MgSO}_{4}$. The solvent was removed under reduced pressure. The crude product was purified by column (hexane:EtOAc, 3:7) giving 2d $(72 \%, 0.244 \mathrm{~g}, 0.98 \mathrm{mmol})$ as a yellow solid, trans-3d $(10 \%, 0.035 \mathrm{~g}, 0.14 \mathrm{mmol})$ as a solid and cis-3d (7\%, $0.025 \mathrm{~g}, 0.10 \mathrm{mmol}$ ) as a solid. 1-(Dimethoxymethyl)5-acetamido-indane (2d): $\mathrm{mp} 68.4-69.3{ }^{\circ} \mathrm{C}$; IR $v_{\max } / \mathrm{cm}^{-1}$ (film) 828, 1058, 1124, 1372, 1426, 1492, 1546, 1602, 
1667; ${ }^{1} \mathrm{H}$ NMR $\left(200 \mathrm{MHz}, \mathrm{CDCl}_{3}\right) \delta 1.85-2.28(\mathrm{~m}, 3 \mathrm{H})$, 2.13 (s, 3H), 2.70-2.90 (m, 2H), 3.37 (s, 3H), 3.41 (s, 3H), 4.27 (d, 1H, J 7.4 Hz), 7.16 (dd, 1H, J 1.4, 8.2 Hz), 7.32 (d, 1H, J $8.0 \mathrm{~Hz}), 7.46(\mathrm{~s}, 1 \mathrm{H}), 7.84(\mathrm{~s}, 1 \mathrm{H}) ;{ }^{13} \mathrm{C} \mathrm{NMR}$ $\left(75 \mathrm{MHz}, \mathrm{CDCl}_{3}\right) \delta 24.3,27.5,31.4,47.0,52.9,54.2$, 107.2, 116.4, 118.2, 125.6, 136.8, 138.7, 145.6, 168.6; LRMS ( $/ z, \%$ \%) $249\left(\mathrm{M}^{+\bullet}, 2.4 \%\right), 218$ (6), $186(3), 174$ (4), 144 (6), 132 (13), 115 (5), 103 (3), 75 (100); HRMS (m/z) calcd. for $\mathrm{C}_{14} \mathrm{H}_{19} \mathrm{NO}_{3}[\mathrm{M}+\mathrm{H}]^{+} 250.1438$, found 250.1440 . $N$-(trans-5,6-dimethoxy-5,6,7,8-tetrahydronaphthalen2-yl)acetamide (trans-3d): $\mathrm{mp} 108.7-110.5^{\circ} \mathrm{C}$; IR $v_{\max } / \mathrm{cm}^{-1}$ (film) 830, 915, 1091, 1331, 1372, 1419, 1505, 1544, 1598, 1614, 1671, 2934, 3302, 3507; ${ }^{1} \mathrm{H}$ NMR (200 MHz, $\left.\mathrm{CDCl}_{3}\right)$ $\delta 1.81-2.18(\mathrm{~m}, 2 \mathrm{H}), 2.13(\mathrm{~s}, 3 \mathrm{H}), 2.58-2.90(\mathrm{~m}, 2 \mathrm{H}), 3.44(\mathrm{~s}$, $3 \mathrm{H}), 3.48(\mathrm{~s}, 3 \mathrm{H}), 3.67-3.74(\mathrm{~m}, 1 \mathrm{H}) 4.21$ (d, 1H, J 4.8 Hz), 7.21-7.27 (m, 2H), $7.34(\mathrm{~s}, 1 \mathrm{H}), 7.56(\mathrm{~s}, 1 \mathrm{H}) .{ }^{13} \mathrm{C} \mathrm{NMR}$ $\left(75 \mathrm{MHz}, \mathrm{CDCl}_{3}\right) \delta 23.3,24.5,25.5,56.5,57.3,77.8,79.2$, $117.5,119.4,130.5,130.6,137.3,137.9,168.3$; LRMS $(\mathrm{m} / \mathrm{z}, \%) 249\left(\mathrm{M}^{+*}, 23 \%\right), 217$ (27), $191(100) ; \operatorname{HRMS}(\mathrm{m} / \mathrm{z})$ calcd. for $\mathrm{C}_{14} \mathrm{H}_{19} \mathrm{NO}_{3}[\mathrm{M}+\mathrm{Na}]^{+} 272.1257$, found 272.1262 . $N$-(cis-5,6-dimethoxy-5,6,7,8-tetrahydronaphthalen-2-yl) acetamide (cis-3d): IR $v_{\max } / \mathrm{cm}^{-1}$ (film) 817, 882, 1081, 1106, 1372, 1419, 1505, 1544, 1602, 1614, 1671, 2933, 3311, 3509; ${ }^{1} \mathrm{H}$ NMR $\left(200 \mathrm{MHz}, \mathrm{CDCl}_{3}\right) \delta$ 1.86-2.30 (m, $2 \mathrm{H}), 2.16$ (s, 3H), 2.68-3.07 (m, 2H), 3.45 (s, 3H), 3.47 (s, $3 \mathrm{H}), 3.57-3.67$ (m, 1H), 4.32 (d, 1H, J 2.8 Hz), 7.25-7.34 $(\mathrm{m}, 4 \mathrm{H}) ;{ }^{13} \mathrm{C} \mathrm{NMR}\left(75 \mathrm{MHz}, \mathrm{CDCl}_{3}\right) \delta 22.3,24.6,27.3$, 56.4, 57.1, 77.5, 78.2, 117.1, 119.8, 130.5, 130.6, 137.6, 137.7, 168.3.; LRMS ( $\mathrm{m} / \mathrm{z}, \%) 249\left(\mathrm{M}^{+*}, 21 \%\right), 217$ (28), $191(100) ; \operatorname{HRMS}(\mathrm{m} / \mathrm{z})$ calcd. for $\mathrm{C}_{14} \mathrm{H}_{19} \mathrm{NO}_{3}[\mathrm{M}+\mathrm{Na}]^{+}$ 272.1257 , found 272.1260 .

Syntheses of cis and trans-1-(2,3)-dihydro-1-metyl1H-inden-3-yl)pentan-1-one (5l)

To a solution of $11(0.129 \mathrm{~g}, 0.647 \mathrm{mmol})$ and molecular sieves $(3 \AA, 0.065 \mathrm{~g})$ in anhydrous $\mathrm{CH}_{3} \mathrm{CN}(6.5 \mathrm{~mL})$ under $\mathrm{N}_{2}$ was added HTIB $(0.489 \mathrm{~g}, 1.25 \mathrm{mmol})$ at $0{ }^{\circ} \mathrm{C}$. The reaction was stirred for $15 \mathrm{~min}$ at $0^{\circ} \mathrm{C}$. A saturated solution of $\mathrm{NaHCO}_{3}$ was added until $\mathrm{pH}$ 7. The organic phase was washed with $\mathrm{H}_{2} \mathrm{O}$, with brine and dried over anhydrous $\mathrm{MgSO}_{4}$. The solvent was removed under reduced pressure. The crude product was purified by column (0-10\% EtOAc in hexane), affording 51 (0.0681 g, $0.315 \mathrm{mmol}, 49 \%)$ as a trans:cis (10:1) mixture. Naphthalene $4 \mathbf{l}^{9}(0.0031 \mathrm{~g}$, $0.016 \mathrm{mmol}, 2 \%$ ) was also isolated as a colorless oil. cis and trans-5l: yellow oil; IR $v_{\max } / \mathrm{cm}^{-1}$ (film) 755, 1460, 1711, 2870, 2931, 2959; ${ }^{1} \mathrm{H}$ NMR (300 MHz, $\left.\mathrm{CDCl}_{3}\right) \delta$ (trans isomer) $0.87(\mathrm{t}, 3 \mathrm{H}, J 7.3 \mathrm{~Hz}), 1.21-1.25(\mathrm{~m}, 1 \mathrm{H}), 1.28(\mathrm{~d}$, $3 \mathrm{H}, J 6.9 \mathrm{~Hz}), 1.51-1.56(\mathrm{~m}, 2 \mathrm{H}), 2.41-2.62(\mathrm{~m}, 3 \mathrm{H}), 3.40$ (sext, 1H, J 6.8 Hz), 4.08 (dd, 1H, J 8.7, 3.4 Hz), 7.14-7.28 (m, 4H), (cis isomer) $0.91(\mathrm{t}, 3 \mathrm{H}, J 7.5 \mathrm{~Hz}), 1.35(\mathrm{~d}, 3 \mathrm{H}$,
$J 6.9 \mathrm{~Hz}$ ), 1.81-1.85 (m, 2H), 3.21 (sext, 1H, J 7.2 Hz) (other signals overlap with the trans form); ${ }^{13} \mathrm{C}$ NMR $(75 \mathrm{MHz}$, $\left.\mathrm{CDCl}_{3}\right) \delta$ (trans isomer) 13.8, 20.2, 22.3, 25.8, 37.8, 38.5, 40.1, 57.0, 123.8, 124.7, 126.6, 127.6, 140.7, 149.3, 210.8, (cis isomer) 13.9, 19.7, 22.4, 25.9, 38.3, 40.8, 123.5, 124.6, 127.4, 141.0, 148.8, 211.3 (other signals overlap with the trans form); LRMS $(\mathrm{m} / z, \%) 216\left(\mathrm{M}^{+\bullet}, 5\right), 131$ (100); HRMS $(\mathrm{m} / z)$ calcd. for $\mathrm{C}_{15} \mathrm{H}_{20} \mathrm{O}[\mathrm{M}+\mathrm{H}]^{+} 217.1587$, found 217.1586 .

Reaction of 4-isopropyl-1-methyl-1,2-dihydronaphthalene (1m) with $\mathrm{HTIB}$ in $\mathrm{CH}_{3} \mathrm{CN}$

The typical procedure for reactions in $\mathrm{CH}_{3} \mathrm{CN}$ was followed, but using $\mathbf{5 m}(0.187 \mathrm{~g}, 1.00 \mathrm{mmol})$. The crude product was purified by flash column chromatography (gradient elution, 0-20\% EtOAc in hexanes), affording indane $5 \mathrm{~m}(0.0981 \mathrm{~g}, 0.485 \mathrm{mmol}, 48 \%)^{13}$ as a trans:cis ( $5: 1$ by ${ }^{1} \mathrm{H}$ NMR after purification) mixture, as yellow oil. Naphthalene 4m (0.0244 g, $0.132 \mathrm{mmol}, 13 \%)^{38}$ was also isolated as colorless oil.

Reaction of 1,2-dihydro-7-methoxy-4-methylnaphthalene (1i) with $\mathrm{HTIB}$ in $\mathrm{CH}_{3} \mathrm{CN}$

To a solution of $\mathbf{1 i}(0.178 \mathrm{~g}, 1.02 \mathrm{mmol})$ and molecular sieves ( $3 \AA, 0.100 \mathrm{~g}$ ) in $\mathrm{CH}_{3} \mathrm{CN}(10 \mathrm{~mL})$ under $\mathrm{N}_{2}$ was added HTIB (0.442 g, $1.13 \mathrm{mmol})$ at $0{ }^{\circ} \mathrm{C}$. The ice bath was removed. The mixture was stirred for $15 \mathrm{~min}$ at room temperature. A saturated solution of $\mathrm{NaHCO}_{3}$ was added until $\mathrm{pH}$ 7. The organic phase was washed with $\mathrm{H}_{2} \mathrm{O}$, with brine and dried over anhydous $\mathrm{MgSO}_{4}$. The solvent was removed under reduced pressure. The crude product was purified by column (0-40\% EtOAc in hexane), affording $\mathbf{5 i}^{39}(0.0231 \mathrm{~g}, 0.121 \mathrm{mmol}, 12 \%)$, as a yellow oil and a mixture $1: 1$ of $4 \mathbf{i}^{40}$ and starting material $(0.0361 \mathrm{~g})$, as a colorless oil.

Reaction of 1,2-dihydro-6-methoxy-4,7-dimethynaphthalene (1j) with $\mathrm{HTIB}$ in $\mathrm{CH}_{3} \mathrm{CN}$

As for 1i, but using $\mathbf{1 j}(0.197 \mathrm{~g}, 1.05 \mathrm{mmol})$, molecular sieves ( $3 \AA, 0.100 \mathrm{~g})$, HTIB (0.489 g, $1.25 \mathrm{mmol})$, $\mathrm{CH}_{3} \mathrm{CN}(10 \mathrm{~mL})$. The mixture was stirred for $30 \mathrm{~min}$ at room temperature. The crude product was purified by column (0-40\% EtOAc in hexane) affording $\mathbf{5} \mathbf{j}^{8}(0.0420 \mathrm{~g}$, $0.204 \mathrm{mmol}, 20 \%)$ and impure $\mathbf{4 j}(0.0594 \mathrm{~g})$. Impure $\mathbf{4 j}$ was purified by column (10\% EtOAc in hexane), affording $\mathbf{4 j}^{41}$ (0.0381 g, $\left.0.205 \mathrm{mmol}, 20 \%\right)$.

Reaction of 1,2-dihydro-1-methyl-4-phenylnaphthalene (1n) with $\mathrm{HTIB}$ in $\mathrm{CH}_{3} \mathrm{CN}$

As for 5l, but using $1 \mathbf{n}(0.165 \mathrm{~g}, 0.750 \mathrm{mmol})$, molecular sieves ( $3 \AA$ A $0.0750 \mathrm{~g}$ ), HTIB (0.353 g, $0.901 \mathrm{mmol})$, and 
$\mathrm{CH}_{3} \mathrm{CN}(7.5 \mathrm{~mL})$. The mixture was stirred for $20 \mathrm{~min}$ at room temperature. The product crude was purified by column (0-10\% EtOAc in hexane) affording $\mathbf{5 n}$ $(0.0452 \mathrm{~g}, 0.191 \mathrm{mmol}, 26 \%),{ }^{13}$ and $6 \mathrm{n}(0.0414 \mathrm{~g}$, $0.175 \mathrm{mmol}, 23 \%),{ }^{18}$ as yellow oil and as a cis:trans mixture (6:1). $4 \mathrm{n}(7.00 \mathrm{mg}, 0.0321 \mathrm{mmol}, 4 \%)^{42}$ was isolated, as a colorless oil.

Reaction of 4-(3,4-dichlorophenyl)-1-methyl-1,2-dihydronaphthalene (1o) with $\mathrm{HTIB}$ in $\mathrm{CH}_{3} \mathrm{CN}$

As for 1i, but using $10(0.118 \mathrm{~g}, 0.408 \mathrm{mmol})$, molecular sieves ( $3 \AA$, $0.0413 \mathrm{~g}$ ), HTIB $(0.194 \mathrm{~g}, 0.495 \mathrm{mmol})$, and $\mathrm{CH}_{3} \mathrm{CN}(4.0 \mathrm{~mL})$. The mixture was stirred for $20 \mathrm{~min}$ at room temperature. The product was purified by column ( 0 $30 \%$ EtOAc in hexane) affording $\mathbf{5 o}(0.0430 \mathrm{~g}, 0.141 \mathrm{mmol}$, $35 \%)$ and $7 \mathbf{o}(0.0070 \mathrm{~g}, 0.023 \mathrm{mmol}, 6 \%)$, both as a yellow oil. trans-(3,4-Dichlorophenyl)-2,3-dihydro-1-methyl$1 H$-inden-3-yl)methanone (5o): IR $v_{\max } / \mathrm{cm}^{-1}$ (film) 755, 1030, 1206, 1687, 2867, 2925, 2958; 'H NMR $(500 \mathrm{MHz}$, $\left.\mathrm{CDCl}_{3}\right) \delta 1.34(\mathrm{~d}, 3 \mathrm{H}, J 6.9 \mathrm{~Hz}), 2.0 .2(\mathrm{ddd}, 1 \mathrm{H}, J 12.8$, 7.6, $8.7 \mathrm{~Hz}$ ), 2.68 (ddd, 1H, $J$ 12.5, 7.8, $4.0 \mathrm{~Hz}$ ), 3.49 (sext, 1H, J 7.2 Hz), 4.95 (dd, 1H, J 8.8, 3.9 Hz), 7.04 (d, 1H, J 7.5 Hz), 7.11-7.14 (m, 1H), 7.24-7.25 (m, 2H), 7.59 (d, 1H, $J 8.4 \mathrm{~Hz}), 7.86(\mathrm{dd}, 1 \mathrm{H}, J 8.3,2.0 \mathrm{~Hz}), 8.11$ $(\mathrm{d}, 1 \mathrm{H}, J 2.0 \mathrm{~Hz}) ;{ }^{13} \mathrm{C}$ NMR $\left(75 \mathrm{MHz}, \mathrm{CDCl}_{3}\right) \delta 20.4$, 38.4, 38.5, 51.3, 124.0, 124.0, 126.6, 127.9, 127.9, 130.8, 130.8, 133.5, 136.4, 137.7, 140.0, 149.4, 198.2; LRMS $(\mathrm{m} / \mathrm{z}, \%) 305\left(\mathrm{M}^{+*}, 1 \%\right), 131(100)$; HRMS $(\mathrm{m} / \mathrm{z})$ calcd. for $\mathrm{C}_{17} \mathrm{H}_{14} \mathrm{Cl}_{2} \mathrm{O}[\mathrm{M}+\mathrm{H}]^{+}$305.0494, found 305.0486. 1-(3,4-Dichlorophenyl)-3,4-dihydro-4-methylnaphthalen2(1H)-one (7o): IR $v_{\max } / \mathrm{cm}^{-1}$ (film) 755, 1030, 1206, 1687, 2867, 2925, 2958; 760, 1031, 1175, 1380, 1467, 1722, 2928, 2963; ${ }^{1} \mathrm{H}$ NMR (300 MHz, $\left.\mathrm{CDCl}_{3}\right) \delta 2.31(\mathrm{dd}, 1 \mathrm{H}$, $J$ 16.8, $7.5 \mathrm{~Hz}), 2.98$ (dd, 1H, J 16.8, $6.6 \mathrm{~Hz}), 3.12$ (sext, $1 \mathrm{H}, J 6.9 \mathrm{~Hz}), 4.77(\mathrm{~s}, 1 \mathrm{H}), 6.88(\mathrm{dd}, 1 \mathrm{H}, J 8.4,2.4 \mathrm{~Hz})$, 7.16 (d, 1H, J 2.1 Hz), 7.30-7.47 (m, 4H), 7.62-7.71 (m, $1 \mathrm{H},) ;{ }^{13} \mathrm{C}$ NMR $\left(75 \mathrm{MHz}, \mathrm{CDCl}_{3}\right) \delta 20.1,32.5,41.8,54.2$, $125.5,126.5,127.5,127.8,128.9,129.2,130.6,132.8$, 133.9, 137.2, 140.2, 141.1, 208.8; HRMS $(\mathrm{m} / \mathrm{z})$ calcd. for $\mathrm{C}_{17} \mathrm{H}_{14} \mathrm{Cl}_{2} \mathrm{O}[\mathrm{M}+\mathrm{H}]^{+}$305.0494, found 305.0486.

\section{Reaction of 9-methyl-6,7-dihydro-5H-benzo[7]annulene (1p) with $\mathrm{HTIB}$ in $\mathrm{CH}_{3} \mathrm{CN}$}

The typical procedure for reactions in $\mathrm{CH}_{3} \mathrm{CN}$ was followed, but using $1 \mathbf{p}(0.0416 \mathrm{~g}, 0.263 \mathrm{mmol})$, molecular sieves $(3 \AA$. $0.0179 \mathrm{~g})$, HTIB $(0.118 \mathrm{~g}, 0.301 \mathrm{mmol})$ in anhydrous $\mathrm{CH}_{3} \mathrm{CN}(2.5 \mathrm{~mL})$. The mixture was stirred for $15 \mathrm{~min}$ at room temperature. The crude product was purified by flash column chromatography (15\% EtOAc in hexanes) affording $\mathbf{5} \mathbf{p}^{43}(0.0241 \mathrm{~g}, 0.138 \mathrm{mmol}, 52 \%)$, as a colorless oil.
Synthesis of 1-[bis(trifluoromethoxy)methyl]-2,3-dihydro$1 \mathrm{H}$-indene $(8 \mathrm{a})$

To a stirred mixture of $\mathbf{1 a}(0.102 \mathrm{~g}, 0.78 \mathrm{mmol})$ and TFE $(6 \mathrm{~mL})$, was added HTIB $(0.34 \mathrm{~g}, 0.86 \mathrm{mmol})$ at once at $0{ }^{\circ} \mathrm{C}$. After $30 \mathrm{~min}$ the reaction was quenched with saturated solution of $\mathrm{NaHCO}_{3}$ until $\mathrm{pH}$ 7. The aqueous phase was extracted with EtOAc $(3 \times 10 \mathrm{~mL})$, washed with brine $(2 \times 10 \mathrm{~mL})$ and dried over anhydrous $\mathrm{MgSO}_{4}$. The solvent was removed under reduced pressure. The crude product was purified by column (hexane:EtOAc, 9:1) giving 8a (73\%, $0.19 \mathrm{~g}, 0.57 \mathrm{mmol})$ as a light yellow oil; IR $v_{\text {max }} / \mathrm{cm}^{-1}$ (film) 2949, 2855, 1460, 1281, 1164, 1078; ${ }^{1} \mathrm{H}$ NMR $\left(300 \mathrm{MHz}, \mathrm{CDCl}_{3}\right) \delta 1.96-2.08(\mathrm{~m}, 1 \mathrm{H}), 2.18-2.30$ (m, 1H), 2.82-3.03 (m, 2H), 3.47 (q, 1H, J 7.9 Hz), 3.864.07 (m, 4H), 4.70 (d, 1H, J 7.9 Hz), 7.15-7.24 (m, 3H), 7.38-7.41 (m, $1 \mathrm{H}) ;{ }^{13} \mathrm{C}$ NMR $\left(75 \mathrm{MHz}, \mathrm{CDCl}_{3}\right) \delta 27.1$, 31.2, 47.2, 61.9 (q, J 34.9 Hz), 63.4 (q, J 34.9 Hz), 105.4, 123.7 (q, $J 276 \mathrm{~Hz}), 123.8(\mathrm{q}, J 276 \mathrm{~Hz}), 124.6,125.5$, 126.5, 127.6, 140.8, 144.6; LRMS $(\mathrm{m} / \mathrm{z}, \%) 328\left(\mathrm{M}^{+}\right.$, 1.3\%), 211 (70), 129 (21), 117 (100); HRMS ( $\mathrm{m} / \mathrm{z}$ ) calcd. for $\mathrm{C}_{14} \mathrm{H}_{14} \mathrm{~F}_{6} \mathrm{O}_{2}[\mathrm{M}+\mathrm{Na}]^{+}$351.0790, found 351.0801.

Synthesis of 1-[bis(trifluoromethoxy)methyl]-2,3-dihydro3-methyl-1H-indene (8b)

As for 1a, but using $1 \mathbf{b}(0.146 \mathrm{~g}, 1.01 \mathrm{mmol})$. HTIB was added at once. The reaction was quenched after 7 min. Compound $\mathbf{8 b}$ was obtained as a yellow oil $(70 \%, 0.243 \mathrm{~g}, 0.710 \mathrm{mmol})$ as a $5: 1$ trans:cis mixture; IR $v_{\text {max }} / \mathrm{cm}^{-1}$ (film) 2961, 2932, 2872, 1458, 1281, 1174, 1078,$758 ;{ }^{1} \mathrm{H}$ NMR $\left(300 \mathrm{MHz}, \mathrm{CDCl}_{3}\right) \delta$ (trans isomer) 1.27 (d, $3 \mathrm{H}, J 6.9 \mathrm{~Hz}), 1.81$ (ddd, $1 \mathrm{H}, J 13.2,8.5$, $7.3 \mathrm{~Hz}), 2.32(\mathrm{ddd}, 1 \mathrm{H}, J 13.2,7.8,4.2 \mathrm{~Hz}), 3.22-3.34$ $(\mathrm{m}, 1 \mathrm{H}), 3.43-3.50(\mathrm{~m}, 1 \mathrm{H}), 3.82-4.06(\mathrm{~m}, 4 \mathrm{H}), 4.64(\mathrm{~d}$, $1 \mathrm{H}, J 8.1 \mathrm{~Hz}), 7.18-7.36(\mathrm{~m}, 4 \mathrm{H})$, (cis isomer) $1.33(\mathrm{~d}$, $3 \mathrm{H}, J 6.9 \mathrm{~Hz}), 2.40-2.55(\mathrm{~m}, 1 \mathrm{H}), 4.75(\mathrm{~d}, 1 \mathrm{H}, J 8.4 \mathrm{~Hz})$, 7.08-7.13 (m, 1H), 7.41-7.44 (m, 1H), 7.68-7.72 (m, 1H) (other signals overlap with the trans form); ${ }^{13} \mathrm{C}$ NMR $\left(75 \mathrm{MHz}, \mathrm{CDCl}_{3}\right) \delta$ (trans isomer) 20.5, 35.9, 37.6, 46.0, $61.6(\mathrm{q}, J 34.7 \mathrm{~Hz}), 63.8$ (q, J34.7 Hz), 105.1, 123.5, 123.6 $(\mathrm{q}, J 276 \mathrm{~Hz}), 123.8$ (q, $J 276 \mathrm{~Hz}), 125.8,126.6,127.8$, 140.3, 149.2, (cis isomer) 19.7, 36.9, 37.7, 45.6, 105.7, 123.3, 124.8, 126.7, 127.6, 130.2, 137.5 (other signals overlap with the trans form); LRMS $(\mathrm{m} / \mathrm{z}, \%)$ (major diastereomer) $242\left(\mathrm{M}^{+\bullet}-\mathrm{CF}_{3} \mathrm{CH}_{2} \mathrm{OH}, 17 \%\right), 211$ (69), 131 (100), (minor diastereomer) $342\left(\mathrm{M}^{+\bullet}, 3 \%\right), 242(9)$, 211 (75), 131 (100); HRMS ( $/ / z)$ calcd. for $\mathrm{C}_{15} \mathrm{H}_{16} \mathrm{~F}_{6} \mathrm{O}_{2}$ $[\mathrm{M}+\mathrm{Na}]^{+}$365.0947, found 365.0960.

Synthesis of 1-(2,3-dihydro-1H-inden-3-yl)ethanone (5h)

As for 1a, but using $\mathbf{1 h}(0.158 \mathrm{~g}, 1.10 \mathrm{mmol})$. The reaction was quenched after $30 \mathrm{~min}$. The crude product was 
purified by column (5-10\% EtOAc in hexane) affording $\mathbf{5} \mathbf{h}^{7}$ $(72 \%, 0.127 \mathrm{~g}, 0.791 \mathrm{mmol})$, as a light yellow oil.

\section{Synthesis of 1-(1,2,3,4-tetrahydronaphthalene-4-yl)} ethanone (5p)

As for 1a, but using 1p $(0.158 \mathrm{~g}, 1.00 \mathrm{mmol})$. The reaction was quenched after $20 \mathrm{~min}$. The crude product was purified using column (hexane:EtOAc, 9:1) giving $\mathbf{5} \mathbf{p}^{13}$ (62\%, $0.107 \mathrm{~g}, 0.62 \mathrm{mmol})$, as a light yellow oil.

Synthesis of 1-(bis(2,2,2-trifluoroethoxy)methyl)3-(4-fluorophenyl)-2,3-dihydro-1H-indene (8q)

A dry round bottom flask was charged with 1q (240 mg, $1.07 \mathrm{mmol}), \mathrm{CH}_{2} \mathrm{Cl}_{2} / \mathrm{TFE}(4: 1 \mathrm{v} / \mathrm{v})$ followed by HTIB $(550 \mathrm{mg}, 1.40 \mathrm{mmol})$ at room temperature. The reaction color changed towards yellow within a minute. After $10 \mathrm{~min}$ at room temperature, the reaction was quenched with $\mathrm{H}_{2} \mathrm{O}$, washed with $\mathrm{H}_{2} \mathrm{O}(2 \times 20 \mathrm{~mL})$, with $50 \% \mathrm{NaHCO}_{3}$ solution $(2 \times 20 \mathrm{~mL})$, with $\mathrm{H}_{2} \mathrm{O}(20 \mathrm{~mL})$, with brine $(2 \times 20 \mathrm{~mL})$, dried over anhydrous $\mathrm{Na}_{2} \mathrm{SO}_{4}$, and filtered. The solvent was removed under reduced pressure to give a brownish oil. The crude product was purified by column (0-25\% EtOAc in hexane) giving 8q (312 mg, $0.739 \mathrm{mmol}, 69 \%)$, as a trans:cis (17:1) mixture as colorless oil; ${ }^{1} \mathrm{H}$ NMR (300 MHz, $\mathrm{CDCl}_{3}$ ) $\delta$ (trans isomer) 2.18 (ddd, $1 \mathrm{H}, 13.5,8.5,7.7 \mathrm{~Hz}), 2.60$ (ddd, $1 \mathrm{H}, J 13,5,8.2,4.2 \mathrm{~Hz}$ ), $3.58(\mathrm{dt}, 1 \mathrm{H}, J 8.2,4.2 \mathrm{~Hz}), 4.11-3.80(\mathrm{~m}, 4 \mathrm{H}), 4.43(\mathrm{t}$, $1 \mathrm{H}, J 8.0 \mathrm{~Hz}), 4.74(\mathrm{~d}, 1 \mathrm{H}, J 7.9 \mathrm{~Hz}), 7.03-6.93(\mathrm{~m}, 3 \mathrm{H})$, 7.10-7.03 (m, 2H), 7.29-7.20 (m, 2H), 7.47-7.39 (m, 1H); ${ }^{13} \mathrm{C} \mathrm{NMR}\left(125 \mathrm{MHz}, \mathrm{CDCl}_{3}\right) \delta$ (trans isomer) 38.0, 46.5, 49.2, 62.0 (q, J $35 \mathrm{~Hz}), 64.1$ (q, J $35 \mathrm{~Hz}), 105.3,115.5$ (d, $J 21 \mathrm{~Hz}), 123.8$ (q, $J 278 \mathrm{~Hz}), 123.9$ (q, J $278 \mathrm{~Hz}), 125.4$, 126.0, 127.4, 128.3, 129.5 (d, J 8 Hz), 140.8 (d, J $3 \mathrm{~Hz}$ ), 141.2, 147.2, $161.8(\mathrm{~d}, J 245 \mathrm{~Hz})$; HRMS $(\mathrm{m} / \mathrm{z})$ calcd. for $\mathrm{C}_{20} \mathrm{H}_{17} \mathrm{~F}_{7} \mathrm{O}_{2}[\mathrm{M}+\mathrm{Na}]^{+}$445.1009, found 445.1017.

Synthesis of 1-(bis(2,2,2-trifluoroethoxy)methyl)2,3-dihydro-1H-indene ( $8 a)$

As for 1q, but using 1a $(0.130 \mathrm{~g}, 1.00 \mathrm{mmol})$. HTIB $(0.510 \mathrm{~g}, 1.30 \mathrm{mmol})$ was added at $0{ }^{\circ} \mathrm{C}$. The reaction was stirred for $10 \mathrm{~min}$ at room temperature. The crude product was purified by column (1-10\% EtOAc in hexane) affording 8a $(67 \%, 0.221 \mathrm{~g}, 0.673 \mathrm{mmol})$, as a yellow oil.

Synthesis of 1-(2,3-dihydro-1-methyl-1H-inden-3-yl) ethanone $(5 \boldsymbol{k})$

As for 1q, but using $1 \mathbf{k}(0.158 \mathrm{~g}, 1.00 \mathrm{mmol})$. HTIB (1.3 equiv.) was added at $0{ }^{\circ} \mathrm{C}$. The reaction was stirred for $10 \mathrm{~min}$ at room temperature. The crude product was purified by column (1-20\% EtOAc in hexane) affording $\mathbf{5} \mathbf{k}^{7}(76 \%$, $0.132 \mathrm{~g}, 0.758 \mathrm{mmol})$, as a light yellow oil.
Synthesis of 1-(2,3-dihydro-1-methyl-1H-inden-3-yl)2-methylpropan-1-one (5m)

As for 1q, but using $\mathbf{1 m}(0.108 \mathrm{~g}, 0.580 \mathrm{mmol})$. HTIB (1.3 equiv.) was added at $0{ }^{\circ} \mathrm{C}$ and the reaction was quenched after $2 \min$ at $0{ }^{\circ} \mathrm{C}$. The crude product was purified by column (2-30\% EtOAc in hexane) affording $\mathbf{5 m}^{13}(62 \%, 0.073 \mathrm{~g}, 0.359 \mathrm{mmol})$, as a light yellow oil.

Synthesis of 2,3-dihydro-1H-indene-1-carbaldehyde (5a)

To a stirred solution of $1 \mathbf{a}(0.122 \mathrm{~g}, 0.937 \mathrm{mmol})$ in HFIP $(4.0 \mathrm{~mL})$ was added HTIB $(0.404 \mathrm{~g}, 1.04 \mathrm{mmol})$ at $0{ }^{\circ} \mathrm{C}$. After $1 \mathrm{~min}$ the reaction was quenched with saturated solution of $\mathrm{Na}_{2} \mathrm{~S}_{2} \mathrm{O}_{3}(5.0 \mathrm{~mL})$. The resulting mixture was extracted with EtOAc $(3 \times 10 \mathrm{~mL})$. The organic layer was washed with brine and dried over anhydrous $\mathrm{MgSO}_{4}$. The solvent was removed under reduce pressure and the crude product was purified by column (5-10\% EtOAc in hexane) giving $\mathbf{5} \mathbf{a}^{13}(58 \%, 0.080 \mathrm{~g}, 0.55 \mathrm{mmol})$ as a light yellow oil.

Reaction of 1,2-dihydronaphthalene (1a) with HTIB in $\mathrm{HFIP} / \mathrm{CH}_{2} \mathrm{Cl}_{2}$ followed by in situ reduction with $\mathrm{NaBH}_{4}$

To a stirred solution of $\mathbf{1 a}(0.050 \mathrm{~g}, 0.38 \mathrm{mmol})$ in HFIP $(0.8 \mathrm{~mL})$ and $\mathrm{CH}_{2} \mathrm{Cl}_{2}(3.2 \mathrm{~mL})$ was added at $0{ }^{\circ} \mathrm{C}$ HTIB $(0.19 \mathrm{~g}, 0.49 \mathrm{mmol})$. The mixture was stirred for 15 min. Then, $\mathrm{NaBH}_{4}(0.72 \mathrm{~g}, 1.9 \mathrm{mmol})$ was added and the reaction was allowed to reach room temperature while stirring for $20 \mathrm{~min}$. Alcohol 9a was obtained as a mixture with ditosilate 10a as a yellow oil after column chromatography (AcOEt in hexanes, 1 to $30 \%$ ). A second column chromatography (20\% AcOEt in hexanes) allowed complete separation of the products giving $9 \mathbf{a}^{44}(48 \%$, $0.027 \mathrm{~g}, 0.18 \mathrm{mmol})$ as a yellow oil and $\mathbf{1 0 a}(17 \%, 0.031 \mathrm{~g}$, $0.066 \mathrm{mmol})$ as a white solid. (2,3-dihydro- $1 H$-inden1-yl)methylene bis(4-methylbenzenesulfonate) (10a): IR $v_{\text {max }} / \mathrm{cm}^{-1}$ (film) 1376, 1193, 1178, $750 \mathrm{~cm}^{-1} ;{ }^{1} \mathrm{H} \mathrm{RMN}$ $\left(200 \mathrm{MHz}, \mathrm{CDCl}_{3}\right) \delta$ 2.10-2.21 (m, 2H), $2.41(\mathrm{~s}, 3 \mathrm{H})$, $2.44(\mathrm{~s}, 3 \mathrm{H}), 2.75-2.87(\mathrm{~m}, 2 \mathrm{H}), 3.55-3.64(\mathrm{~m}, 1 \mathrm{H}), 6.50$ (d, J 3.8 Hz, 1,H), 6.99-7.20 (m, 6H), 7.28-7.32 (m, 2H), 7.44-7.50 (m, 2H), 7.71-7.77 (m, 2H); ${ }^{13} \mathrm{C}$ RMN (75 MHz, $\left.\mathrm{CDCl}_{3}\right) \delta 21.7,21.7,25.3,31.3,50.2,100.6,124.7,125.2$, 126.3, 127.8, 128.1, 129.6, 129.7, 133.2, 133.5; 138.5, 145.0, 145.1, 145.2; HRMS (m/z) calcd. for $\mathrm{C}_{24} \mathrm{H}_{24} \mathrm{O}_{6} \mathrm{~S}_{2}$ $[\mathrm{M}+\mathrm{Na}]^{+}$495.0907, found 495.0910.

\section{Synthesis of (2,3-dihydro-1H-inden-1-yl)methanol (9a)}

To a stirred mixture of $\mathbf{1 a}(0.13 \mathrm{~g}, 1.0 \mathrm{mmol})$ and $\mathrm{H}_{2} \mathrm{O}$ ( $0.40 \mathrm{~mL}, 22 \mathrm{mmol})$ was added $\mathrm{CH}_{2} \mathrm{Cl}_{2} / \mathrm{HFIP}(16 \mathrm{~mL} / 4 \mathrm{~mL})$ at $0{ }^{\circ} \mathrm{C}$. HTIB $(0.51 \mathrm{~g}, 1.3 \mathrm{mmol})$ was added dropwise. The mixture was stirred for $5 \mathrm{~min}$ at the same temperature. $\mathrm{NaBH}_{4}$ was added $(0.19 \mathrm{~g}, 5.0 \mathrm{mmol})$ at room temperature. The mixture was stirred for 70 min and $\mathrm{H}_{2} \mathrm{O}$ was added. The 
resulting mixture was extracted with EtOAc. The organic layer was washed with brine and dried over anhydrous $\mathrm{MgSO}_{4}$. The solvent was removed under reduce pressure and the crude product was purified by column $(0-20 \%$ EtOAc in hexane) giving $\mathbf{9 a} \mathrm{a}^{44}(0.109 \mathrm{~g}, 0.736 \mathrm{mmol}, 74 \%)$ as a light yellow oil.

\section{Supplementary Information}

Supplementary information concerning spectroscopic data, experimental procedures and NMR copies are available free of charge at http://jbcs.sbq.org.br as PDF file.

\section{Acknowledgments}

The authors wish to thank The Swedish Foundation for International Cooperation in Research and Higher Education (STINT), Fundação de Amparo à Pesquisa do Estado de São Paulo (FAPESP), Conselho Nacional de Desenvolvimento Científico e Tecnológico (CNPq) and Coordenação de Aperfeiçoamento de Pessoal de Nível Superior (CAPES) for financial support. We thank F. Y. M. Vieira and E. C. Pedrozo for initial experiments.

\section{References}

1. For some selected examples, see: Rizzo, S.; Bartolini, M.; Ceccarini, L.; Piazzi, L.; Gobbi, S.; Cavalli, A.; Recanatini, M.; Andrisano, V.; Rampa, A.; Bioorg. Med. Chem. 2010, 18, 1749; Li, M.; Xia, L.; Chem. Biol. Drug Des. 2007, 70, 461; Dorsey, B. D.; Levin, R. B.; McDaniel, S. L.; Vacca, J. P.; Guare, J. P.; Darke, P. L.; Zugay, J. A.; Emini, E. A.; Schleif, W. A.; Quintero, J. C.; Lin, J. H.; Chen, I.-W.; Holloway, M. K.; Fitzgerald, P. M. D.; Axel, M. G.; Ostovic, D.; Anderson, P. S.; Huff, J. R.; J. Med. Chem. 1994, 37, 3343; Sugimoto, H.; Iimura, Y.; Yamanishi, Y.; Yamatsu, K.; J. Med. Chem. 1995, 38, 4821; Kwiatkowska, A.; Sleszynska, M.; Derdowska, I.; Prahl, A.; Sobolewski, D.; Borovicková, L.; Slaninová, J.; Lammek, B.; J. Pept. Sci. 2010, 16, 15; Corbett, J. W.; Rauckhorst, M. R.; Qian, F.; Hoffman, R. L.; Knauer, C. S.; Fitzgerald, L. W.; Bioorg. Med. Chem. 2007, 17, 6250; Wickens, P.; Zhang, C.; Ma, X.; Zhao, Q.; Amatruda, J.; Bullock, W.; Burns, M.; Cantin, L.-D.; Chuang, C.-Y.; Claus, T.; Dai, M.; Cruz, F. D.; Dickson, D.; Ehrgott, F. J.; Fan, D.; Heald, S.; Hetemann, M.; Iwuagwu, C. I.; Johnson, J. S.; Kumarasinghe, E.; Ladner, D.; Lavoie, R.; Liang, S.; Livingston, J. N.; Lowe, D.; Magnuson, S.; Mannely, G.; Mugge, I.; Ogutu, H.; Williams-Pleasic, S.; Schoenleber, R. W.; Shapiro, J.; Shelekhin, T.; Sweet, L.; Town, C.; Tsutsumi, M.; Bioorg. Med. Chem. 2007, 17, 4369; Kato, S.; Tanaka, A.; Watanabe, H.; Sato, Y.; Ikeda, Y.; Böger, P.; Wakabayashi, K.; J. Pestic. Sci. 2005, 30, 7.
2. For some recent examples, see: Zhang, L.; Herndon, J. W.; Organometallics 2004, 23, 1231; Chen, W.-F.; Lin, H.-Y.; Dai, S. A.; Org. Lett. 2004, 6, 2341; Kaim, L. E.; Grimaud, L.; Vieu, E.; Org. Lett. 2007, 9, 4171; Kesavan, S.; Panek, J. S.; Porco Jr., J. A.; Org. Lett. 2007, 9, 5203; Watson, M. P.; Jacobsen, E. N.; J. Am. Chem. Soc. 2008, 130, 12594; Sánchez-Larios, E.; Gravel, M.; J. Org. Chem. 2009, 74, 7536; Pi, S. F.; Yang, X. H.; Huang, X. C.; Liang, Y.; Yang, G. N.; Zhang, X. H.; Li, J. H.; J. Org. Chem. 2010, 75, 3484; Wilsily, A.; Fillion, E.; J. Org. Chem. 2009, 74, 8583; Ferreira, S. B.; Kaiser, C. R.; Ferreira, V. F.; Synlett 2008, 2625; Minatti, A.; Zheng, X. L.; Buchwald, S. L.; J. Org. Chem. 2007, 72, 9253; Gharpure, S. J.; Reddy, S. R. B.; Sanyal, U.; Synlett 2007, 1889; Wang, Y.; Wu, J.; Xia, P.; Synth. Commun. 2006, 36, 2685; Fillion, E.; Wilsily, A.; J. Am. Chem. Soc. 2006, 128, 2774; Bianco, G. G.; Ferraz, H. M. C.; Costa, A. M.; Costa-Lotufo, L. V.; Pessoa, C.; de Moraes, M. O.; Schrems, M. G.; Pfaltz, A.; Silva Jr., L. F.; J. Org. Chem. 2009, 74, 2561; Ferraz, H. M. C.; Aguilar, A. M.; Silva Jr., L. F.; Tetrahedron 2003, 59, 5817; for a review, see: Ferraz, H. M. C.; Aguilar, A. M.; Silva Jr., L. F.; Craveiro, M. V.; Quim. Nova 2005, 28, 703.

3. For some recent examples, see: Clark, W. M.; Tickner-Eldridge, A. M.; Huang, G. K.; Pridgen, L. N.; Olsen, M. A.; Mills, R. J.; Lantos, I.; Baine, N. H.; J. Am. Chem. Soc. 1998, 120, 4550; Marcus, A. P.; Sarpong, R.; Org. Lett. 2010, 12, 4560; Vacher, B.; Funes, P.; Chopin, P.; Cussac, D.; Heusler, P.; Tourette, A.; Marien, M.; J. Med. Chem. 2010, 53, 6986; Baur, F.; Beattie, D.; Beer, D.; Bentley, D.; Bradley, M.; Bruce, I.; Charlton, S. J.; Cuenoud, B.; Ernst, R.; Fairhurst, R. A.; Faller, B.; Farr, D.; Keller, T.; Fozard, J. R.; Fullerton, J.; Garman, S.; Hatto, J.; Hayden, C.; He, H. D.; Howes, C.; Janus, D.; Jiang, Z. J.; Lewis, C.; Loeuillet-Ritzler, F.; Moser, H.; Reilly, J.; Steward, A.; Sykes, D.; Tedaldi, L.; Trifilieff, A.; Tweed, M.; Watson, S.; Wissler, E.; Wyss, D.; J. Med. Chem. 2010, 53, 3675; Wood, J. L.; Pujanauski, B. G.; Sarpong, R.; Org. Lett. 2009, 11, 3128; Dinges, J.; Albert, D. H.; Arnold, L. D.; Ashworth, K. L.; Akritopoulou-Zanze, I.; Bousquet, P. F.; Bouska, J. J.; Cunha, G. A.; Davidsen, S. K.; Diaz, G. J.; Djuric, S. W.; Gasiecki, A. F.; Gintant, G. A.; Gracias, V. J.; Harris, C. M.; Houseman, K. A.; Hutchins, C. W.; Johnson, E. F.; Li, H.; Marcotte, P. A.; Martin, R. L.; Michaelides, M. R.; Nyein, M.; Sowin, T. J.; Su, Z.; Tapang, P. H.; Xia, Z. R.; Zhang, H. Q.; J. Med. Chem. 2007, 50, 2011.

4. For a review concerning ring contraction reactions, see: Silva Jr., L. F.; Tetrahedron 2002, 58, 9137.

5. For some recent reviews, see: Stang, P. J.; Zhdankin, V. V.; Chem. Rev. 1996, 96, 1123; Varvoglis, A.; Hypervalent Iodine in Organic Synthesis; Academic Press: London, 1997; Varvoglis, A.; Tetrahedron 1997, 53, 1179; Koser, G. F.; Aldrichim. Acta 2000, 34, 89; Stang, P. J.; J. Org. Chem. 2003, 68, 2997; Koser, G. F.; Aldrichim. Acta 2001, 34, 89; Wirth, T.; Hypervalent Iodine Chemistry; Ed. Springer: Berlin, 2003; Moriarty, R. M.; J. Org. Chem. 2005, 70, 2893; Silva Jr., L. F.; Molecules 
2006, 11, 421; Zhdankin, V. V.; Stang, P. J.; Chem. Rev. 2008, 108, 5299; Merritt, E. A.; Olofsson, B.; Angew. Chem. Int. Ed. 2009, 48, 9052; Merritt, E. A.; Olofsson, B.; Synthesis 2011, 517; Ciufolini, M. A.; Braun, N. A.; Canesi, S.; Ousmer, M.; Chang, J.; Chai, D.; Synthesis 2007, 24, 3759; Zhdankin, V. V.; ARKIVOC 2009, 1; Pouységu, L.; Deffieux, D.; Quideau, S.; Tetrahedron 2010, 66, 2235; Duschek, A.; Kirsch, S. F.; Angew. Chem. Int. Ed. 2011, 50, 1524; Satam, V.; Harad, A.; Rajule, R.; Pati, H.; Tetrahedron 2010, 66, 7659.

6. Justik, M. W.; Koser, G. F.; Tetrahedron Lett. 2004, 45, 6159; Harders, J.; Garming, A.; Jung, A.; Kaiser, V.; Monenschein, H.; Ries, M.; Rose, L. S., K.-U.; Weber, T.; Kirschning, A.; Liebigs Ann. 1997, 2125; Hara, S.; Nakahigashi, J.; Ishi-i, K.; Fukuhara, T.; Yoneda, N.; Tetrahedron Lett. 1998, 39, 2589; Justik, M. W.; Koser, G. F.; Molecules 2005, 10, 217; Kirschning, A.; Eur. J. Org. Chem. 1998, 2267; Moriarty, R. M.; Pakrash, O.; Duncan, M. P.; Vaid, R. K.; Rani, N.; J. Chem. Res. 1996, 432; Zefirov, N. S.; Caple, R.; Palyulin, V. A.; Berglund, B.; Tykvinskii, R.; Zhdankin, V. V.; Kozmin, A. S.; Bull. Acad. Sci. USSR, Div. Chem. Sci. 1988, 37, 1289; Yusubov, M. S.; Zholobova, G. A.; Filimonova, I. L.; Chi, K. W.; Russ. Chem. Bull. 2004, 53, 1735; Koser, G. F.; Rebrovic, L.; Wettach, R. H.; J. Org. Chem. 1981, 46, 4324; Rebrovic, L.; Koser, G. F.; J. Org. Chem. 1984, 49, 2462.

7. For a preliminary communication of part of this work, see: Silva Jr., L. F., Siqueira, F. A.; Pedrozo, E. C.; Vieira, F. Y. M.; Doriguetto, A. C.; Org. Lett. 2007, 9, 1433.

8. Silva Jr., L. F.; Sousa, R. M. F.; Ferraz, H. M. C.; Aguilar, A. M.; J. Braz. Chem. Soc. 2005, 16, 1160.

9. Ferraz, H. M. C.; Silva Jr., L. F.; Vieira, T. O.; Tetrahedron 2001, 57, 1709.

10. For an example, see discussion at Silva Jr., L. F.; Craveiro, M. V.; Org. Lett. 2008, 10, 5417.

11. Miyamoto, K.; Sei, Y.; Yamaguchi, K.; Ochiai, M.; J. Am. Chem. Soc. 2009, 131, 1382.

12. Ochiai, M.; Yoshimura, A.; Miyamoto, K.; Tetrahedron Lett. 2009, 50, 4792.

13. Ferraz, H. M. C.; Carneiro, V. M. T.; Silva Jr., L. F.; Synthesis 2009, 385.

14. Jensen, B. L.; Slobodzian, S. V.; Tetrahedron Lett. 2000, 41, 6029.

15. Banks, H.; Ziffer, H.; J. Org. Chem. 1982, 47, 3743.

16. Ranu, B. C.; Jana, U.; J. Org. Chem. 1998, 63, 8212.

17. Li, L.; Cai, P.; Guo, Q.; Xue, S.; J. Org. Chem. 2008, 73, 3516.

18. Ichikawa, J.; Jyono, H.; Kudo, T.; Fujiwara, M.; Yokota, M.; Synthesis 2005, 39.

19. For seminal papers on the use of TFE in hypervalent iodine oxidations, see: Kita, Y.; Tohma, H.; Kikuchi, K.; Inagaki, M.; Yakura, T.; J. Org. Chem. 1991, 56, 435; Kita, Y.; Yakura, T.; Tohma, H.; Kikuchi, K.; Tamura, Y.; Tetrahedron Lett. 1989, 30,1119 .
20. For an excellent discussion about fluoroalcohols as solvent, see: Dohi, T; Yamaoka, N; Kita, Y.; Tetrahedron 2010, 66, 5775; for other examples, see: Dohi, T.; Maruyama, A.; Takenaga, N.; Senami, K.; Minamitsuji, Y.; Fujioka, H.; Caemmerer, S. B.; Kita, Y.; Angew. Chem. Int. Ed. 2008, 47, 3787; Correa, A.; Tellitu, I.; Dominguez, E.; SanMartin, R.; J. Org. Chem. 2006, 71, 8316; Serna, S.; Tellitu, I.; Dominguez, E.; Moreno, I.; SanMartin, R.; Org. Lett. 2005, 7, 3073; Kita, Y.; Morimoto, K.; Ito, M.; Ogawa, C.; Goto, A.; Dohi, T.; J. Am. Chem. Soc. 2009, 131, 1668; Dohi, T.; Ito, M.; Yamaoka, N.; Morimoto, K.; Fujioka, H.; Kita, Y.; Angew. Chem. Int. Ed. 2010, 49, 3334; Ito, M.; Ogawa, C.; Yamaoka, N.; Fujioka, H.; Dohi, T.; Kita, Y.; Molecules 2010, 15, 1918; Merritt, E. A.; Carneiro, V. M. T.; Silva Jr., L. F.; Olofsson, B.; J. Org. Chem. 2010, 75, 7416.

21. Guérard, K. C.; Chapelle, C.; Giroux, M.-A.; Sabot, C.; Beaulieu, M.-A.; Achache, N.; Canesi, S.; Org. Lett. 2009, 11, 4756.

22. For leading references, see: Andersen, K.; Liljefors, T.; Gundertofte, K.; Perregaard, J.; Bogeso, K. P.; J. Med. Chem. 1994, 37, 950; Bogeso, K. P.; Arnt, J.; Hyttel, J.; Pedersen, H.; J. Med. Chem. 1993, 36, 2761.

23. Biological activity: Richter, J.; Landau, E. M.; Cohen, S.; Mol. Pharmacol. 1977, 13, 548; Cohen, S.; Goldshmid, A.; Shtacher, G.; Srebrenik, S.; Gitter, S.; Mol. Pharmacol. 1975, 11, 379; polymer chemistry: Tomihashi, N.; Yamana, M.; Araki, T.; Eur. Pat. Appl. 1989, EP0 320981A1; Wakabayashi, K.; Tamai, R.; Sekiya, A.; Tamura, M.; Jpn. Kokai Tokkyo Koho 2001, JP 2001031613A.

24. Shipp, K. G.; Hill, M. E.; J. Org. Chem. 1966, 31, 853.

25. Kubota, T.; Miyashita, S.; Kitazume, T.; Ishikawa, N.; J. Org. Chem. 1980, 45, 5052.

26. Patel, N. R.; Chen, J.; Zhang, Y. F.; Kirchmeier, R. L.; Shreeve, J. M.; Inorg. Chem. 1994, 33, 5463.

27. Lucero, M. J.; Houk, K. N.; J. Org. Chem. 1998, 63, 6973.

28. Martinelli, M. J.; Peterson, B. C.; Khau, V. V.; Hutchison, D. R.; Leanna, M. R.; Audia, J. E.; Droste, J. J.; Wu, Y. D.; Houk, K. N.; J. Org. Chem. 1994, 59, 2204.

29. Maeda, K.; Brown, J. M.; Chem. Commun. 2002, 310.

30. Zhdankin, V. V.; Tykwinski, R.; Berglund, B.; Mullikin, M.; Caple, R.; Zefirov, N. S.; Kozmin, A. S.; J. Org. Chem. 1989, $54,2609$.

31. Zefirov, N. S.; Zhdankin, V. V.; Dankov, Y. V.; Sorokin, V. D.; Semerikov, V. N.; Kozmin, A. S.; Caple, R.; Berglund, B. A.; Tetrahedron Lett. 1986, 27, 3971.

32. Zefirov, N. S.; Zhdankin, V. V.; Dankov, Y. V.; Kozmin, A. S.; Zh. Org. Khim. 1984, 20, 446.

33. Zhdankin, V. V.; Kuehl, C. J.; Simonsen, A. J.; J. Org. Chem. 1996, 61, 8272.

34. Muraki, T.; Yokoyama, M.; Togo, H.; J. Org. Chem. 2000, 65, 4679. 
35. For a recent review, see: Silva Jr., L. F.; Carneiro, V. M. T.; Synthesis 2010, 1059.

36. For a review on thallium(III)-mediated ring contractions, see: Ferraz, H. M. C.; Silva Jr., L. F.; Quim. Nova 2000, 23, 216; for examples of Tl(III)-mediated oxidation of 1,2-dihydronaphthalenes, see: Ferraz, H. M. C.; Silva Jr., L. F.; Tetrahedron 2001, 57, 9939; Ferraz, H. M. C.; Aguilar, A. M.; Silva Jr., L. F.; Synthesis 2003, 1031; see also references 2, 8, 9 , and 13; for papers comparing Tl(III) to I(III), see: Prakash, O.; Aldrichim. Acta 1995, 28, 63; Moriarty, R. M.; Khosrowshahi, J. S.; Prakash, O.; Tetrahedron Lett. 1985, 26, 2961.

37. Repinskaya, I. B.; Koryabkin, N. A.; Makarova, Z. S.; Koptyug, V. A.; Zh. Org. Khim. 1982, 18, 870.

38. Kraus, G. A.; Jeon, I.; Org. Lett. 2006, 8, 5315.

39. Fetizon, M.; Moreau, N.; Bull. Soc. Chim. Fr. 1965, 3718.

40. Kasturi, T. R.; Sattigeri, J. A.; Tetrahedron 1992, 48, 6439.
41. Bell, A. A.; Stipanovic, R. D.; Zhang, J.; Mace, M. E.; Phytochemistry 1998, 49, 431.

42. Kabalka, G. W.; Ju, Y.; Wu, Z.; J. Org. Chem. 2003, 68, 7915.

43. Azemi, T.; Kitamura, M.; Narasaka, K.; Tetrahedron 2004, 60, 1339.

44. Goodman, A. L.; Eastman, R. H.; J. Am. Chem. Soc. 1964, 86, 908.

Submitted: March 30, 2011

Published online: July 12, 2011

FAPESP has sponsored the publication of this article. 


\title{
Metal-Free Synthesis of Indanes by Iodine(III)-Mediated Ring Contraction of 1,2-Dihydronaphthalenes
}

\author{
Fernanda A. Siqueira, ${ }^{a, \#}$ Eloisa E. Ishikawa, ${ }^{a}$ André Fogaça, ${ }^{a}$ Andréa T. Faccio, ${ }^{a}$ \\ Vânia M. T. Carneiro, ${ }^{a}$ Rafael R. S. Soares, ${ }^{a, s}$ Aline Utaka, ${ }^{a}$ Iris R. M. Tébéka, ${ }^{a}$ \\ Marcin Bielawski, ${ }^{a, b}$ Berit Olofsson ${ }^{b}$ and Luiz, F. Silva Jr. $* a, s$ \\ ${ }^{a}$ Departamento de Química Fundamental, Instituto de Química, Universidade de São Paulo, \\ CP 26077, 05513-970 São Paulo-SP, Brazil \\ ${ }^{b}$ Department of Organic Chemistry, Arrhenius Laboratory, Stockholm University, \\ SE-106 91 Stockholm, Sweden
}

\section{Experimental}

\section{General information}

HTIB was used as received. Methanol and acetonitrile were distilled from magnesium turnings and $\mathrm{CaH}_{2}$, respectively. These solvents were storaged in a bottle containing $4 \AA$ molecular sieves. THF and $\mathrm{Et}_{2} \mathrm{O}$ were freshly distilled from sodium/benzophenone. Column chromatography was performed using silica gel 200-400 mesh. TLC analyses were performed using silica gel plates, using solutions of phosphomolybdic acid and $p$-anisaldehyde for visualization. NMR spectra were recorded using $\mathrm{CDCl}_{3}$ as solvent and TMS as internal pattern. The substrates $\mathbf{1 a}, \mathbf{1 b}, \mathbf{1 c}, \mathbf{1 e}, \mathbf{1 g}, \mathbf{1 h}, \mathbf{1 j}$ and $\mathbf{1 k}$ were prepared as previously described. ${ }^{1-3}$ See the previous communication for experimental procedures of the HTIB oxidations in $\mathrm{MeOH}$ with 1a, 1b, 1c, $1 \mathbf{d}$ and $\mathbf{1 g}$, and in MeCN with 1a, 19 and $\mathbf{4 l} .^{4}$

Preparation of 1,2-dihydronaphthalenes

\section{7-Acetamido-1,2-dihydronaphthalene (1d)}<smiles>CCNc1ccc2c(c1)CCC=C2</smiles>

In a solution of 6-amino-1-tetralone $(1.00 \mathrm{~g}, 6.21 \mathrm{mmol})$ and DMAP $(0.020 \mathrm{~g})$ in Et3N (25 mL) was added

*e-mail: luizfsjr@iq.usp.br

Present addresses: "Universidade Federal de São Paulo, Campus Diadema, Rua Artur Riedel no. 275, 09972-270, Diadema-SP, Brazil, and \$Instituto Federal de Educação, Ciência e Tecnologia de São Paulo, Rua Pedro Vicente no. 625, São Paulo-SP, Brazil

${ }^{\S}$ Dedicated with deep respect to Prof. Miuako K. Kuya
$\mathrm{Ac}_{2} \mathrm{O}(2.0 \mathrm{~mL})$. The mixture was stirred for $1 \mathrm{~h}$ at room temperature. The reaction was quenched with $\mathrm{MeOH}$ $(10 \mathrm{~mL})$ and $\mathrm{H} 2 \mathrm{O}(15 \mathrm{~mL})$, extracted with EtOAc $(3 \times 15 \mathrm{~mL})$, washed with brine $(2 \times 10 \mathrm{~mL})$ and dried over anhydrous $\mathrm{MgSO}_{4}$. The solvent was removed under reduced pressure and the resulting residue was purified by flash chromatography (silica gel 200-400 mesh, 60\% EtOAc in hexanes) giving 6-acetamido-1-tetralone ${ }^{5}(92 \%, 1.16 \mathrm{~g}$, $5.72 \mathrm{mmol})$ as a light-yellow solid; $\mathrm{mp} 124.5-126.7^{\circ} \mathrm{C}$ $\left(124.5-125^{\circ} \mathrm{C}\right)^{5} ;{ }^{1} \mathrm{H}$ NMR $\left(200 \mathrm{MHz}, \mathrm{CDCl}_{3}\right) \delta 2.02-2.17$ $(\mathrm{m}, 2 \mathrm{H}), 2.22(\mathrm{~s}, 3 \mathrm{H}), 2.62(\mathrm{t}, 2 \mathrm{H}, J 6.5 \mathrm{~Hz}), 2.92(\mathrm{t}, 2 \mathrm{H}$, $J 6.0 \mathrm{~Hz}), 7.27(\mathrm{dd}, 1 \mathrm{H}, J 2.4$ and $8.6 \mathrm{~Hz}), 7.72(\mathrm{~s}, 1 \mathrm{H})$, $7.96(\mathrm{~d}, 1 \mathrm{H}, J 8.4 \mathrm{~Hz}), 8.31(1 \mathrm{H}, \mathrm{s}) ;{ }^{13} \mathrm{C}$ NMR $(75 \mathrm{MHz}$, $\left.\mathrm{CDCl}_{3}\right) \delta 23.2,24.7,29.9,38.9,117.5,118.5,128.4,142.7$, 146.3, 169.0, 197.7.

To a stirred solution of 6-acetamido-1-tetralone $(1.12 \mathrm{~g}$, $5.50 \mathrm{mmol})$ in anhydrous $\mathrm{MeOH}(70 \mathrm{~mL})$ was added $\mathrm{NaBH}_{4}$ $(0.25 \mathrm{~g}, 6.61 \mathrm{mmol})$ in portions at $0{ }^{\circ} \mathrm{C}$. The mixture was stirred for $1 \mathrm{~h}$ at room temperature. The reaction was quenched with $\mathrm{H}_{2} \mathrm{O}(20 \mathrm{~mL})$ and a $10 \%$ aqueous solution of $\mathrm{HCl}$ was added dropwise until $\mathrm{pH} c a$. 7. The resulting solution was extracted with EtOAc $(3 \times 15 \mathrm{~mL})$, washed with brine $(20 \mathrm{~mL})$ and dried over anhydrous $\mathrm{MgSO}_{4}$. The solvent was removed under reduced pressure giving 6-acetamido-1-tetralol $(78 \%, 0.882 \mathrm{mg}, 4.30 \mathrm{mmol})$ as a pale-yellow solid. The 1-tetralol $(0.841 \mathrm{~g}, 4.10 \mathrm{mmol})$ was used without purification in a dehydration reaction using toluene $(45 \mathrm{~mL})$, a few crystals of $p-\mathrm{TsOH}$ and reaction time of $3 \mathrm{~h}$ at $130^{\circ} \mathrm{C}$, using a Dean-Stark apparatus. The resulting residue was purified by flash chromatography (silica gel 200-400 mesh, 80\% EtOAc in hexanes) affording $1 \mathbf{d}^{6}(95 \%$, $0.728 \mathrm{~g}, 3.89 \mathrm{mmol}$ ) as a pale-yellow solid. Experimental data has not been previously reported: $\mathrm{mp}: 89.3-90.6^{\circ} \mathrm{C}$; IR $v_{\text {max }} / \mathrm{cm}^{-1}$ (film) 497, 566, 684, 834, 883, 1018, 1266, 1328, 1370, 1421, 1536, 1594, 1666, 2829, 2883, 2933, 3032, 
3297; ${ }^{1} \mathrm{H}$ NMR (200 MHz, $\mathrm{CDCl}_{3}$ ) $\delta 2.14$ (s, 3H), 2.20-2.31 $(\mathrm{m}, 2 \mathrm{H}), 2.72(\mathrm{t}, 2 \mathrm{H}, J 8.1 \mathrm{~Hz}), 5.90-5.99(\mathrm{~m}, 1 \mathrm{H}), 6.40(\mathrm{~d}$, $1 \mathrm{H}, J 9.6 \mathrm{~Hz}), 6.92(\mathrm{~d}, 1 \mathrm{H}, J 8.0 \mathrm{~Hz}), 7.23(\mathrm{dd}, 1 \mathrm{H}, J 2.2$ and $8.0 \mathrm{~Hz}), 7.31(\mathrm{~s}, 1 \mathrm{H}), 7.89(\mathrm{~s}, 1 \mathrm{H}) ;{ }^{13} \mathrm{C} \mathrm{NMR}(75 \mathrm{MHz}$, $\left.\mathrm{CDCl}_{3}\right) \delta 22.9,24.4,27.6,117.8,119.4,126.1,127.0,127.6$, $130.4,136.3,136.5,168.6$; LRMS $m / z(\%) 187\left(\mathrm{M}^{+*}, 72 \%\right)$, 146 (9), 145 (61), 144 (100), 130 (29), 115 (24), 91 (8), 77 (6), 51 (5), 43 (23); HRMS ( $\mathrm{m} / \mathrm{z}$ ) calcd. for $\mathrm{C}_{12} \mathrm{H}_{13} \mathrm{NO}$ $[\mathrm{M}+\mathrm{H}]^{+} 188.1070$, found 188.1067.

\section{1,2-Dihydro-6-methoxynaphthalene (1f)}<smiles>COc1ccc2c(c1)C=CCC2</smiles>

$\mathrm{NaBH}_{4}(0.455 \mathrm{~g}, 12.0 \mathrm{mmol})$ was added dropwise to a solution of 7-methoxy-1-tetralone $(1.52 \mathrm{~g}, 8.63 \mathrm{mmol})$ in $\mathrm{MeOH}(50 \mathrm{~mL})$ at $0{ }^{\circ} \mathrm{C}$. The mixture was stirred at room temperature. After $2 \mathrm{~h}$, the reaction was quenched with $\mathrm{H}_{2} \mathrm{O}$ and a $10 \%$ aqueous solution of $\mathrm{HCl}$ was added dropwise until $\mathrm{pH} c a$. 5. The $\mathrm{MeOH}$ was removed under reduced pressure and the residue was extracted with EtOAc, washed with brine, and dried over anhydrous $\mathrm{MgSO}_{4}$. The solvent was removed under reduced pressure. The crude corresponding 1-tetralol was dissolved in THF $(10 \mathrm{~mL})$ and $\mathrm{H}_{3} \mathrm{PO}_{4} 85 \%$ (4.5 mL) was added dropwise at room temperature. The mixture was refluxed at $95^{\circ} \mathrm{C}$ for $2 \mathrm{~h}$. The crude product was transferred to an Erlenmeyer and diluted with $\mathrm{Et}_{2} \mathrm{O}$. A sat. solution of $\mathrm{NaHCO}_{3}$ was added until $c a$. $\mathrm{pH}$ 7. The solution was extracted with $\mathrm{Et}_{2} \mathrm{O}$, washed with sat. solution of $\mathrm{NaCl}$ and dried over anhydrous $\mathrm{MgSO}_{4}$. The residue was purified by flash column chromatography (gradient elution, 0-30\% EtOAc in hexanes), affording $\mathbf{1 f}^{2}(0.885 \mathrm{~g}, 5.52 \mathrm{mmol}, 64 \%)$, as a colorless oil. Starting material was also recovered $(0.0089 \mathrm{~g}, 0.0555 \mathrm{mmol}, 1 \%)$, as a colorless oil.

\section{1,2-Dihydro-7-methoxy-4-methylnaphthalene (1i)}<smiles>COc1ccc2c(c1)CCC=C2C</smiles>

A solution of 6-methoxy-1-tetralone (1.76 g, $10.0 \mathrm{mmol})$ in $\mathrm{Et}_{2} \mathrm{O}(7.0 \mathrm{~mL})$ was added to a solution of $\mathrm{MeMgI}$ [prepared from MeI $(1.7 \mathrm{~mL}, 27.0 \mathrm{mmol}), \mathrm{Mg}(0.673 \mathrm{~g}$, $27.7 \mathrm{mmol}$ ) and $\mathrm{I}_{2}$ (some crystals) in anhydrous $\mathrm{Et}_{2} \mathrm{O}$ $(7.0 \mathrm{~mL})]$. The mixture was refluxed for $4.5 \mathrm{~h}$. After that, a solution of $\mathrm{HCl} 6 \mathrm{~mol} \mathrm{~L}^{-1}(6 \mathrm{~mL})$ was added dropwise at $0{ }^{\circ} \mathrm{C}$. The solution was stirred for $15 \mathrm{~min}$ at room temperature. The organic layer was extracted with $\mathrm{Et}_{2} \mathrm{O}$, washed with brine and dried over anhydrous $\mathrm{MgSO}_{4}$. The solvent was removed under reduced pressure. The crude product was purified by flash column chromatography (gradient elution, 0-30\% of EtOAc in hexanes), affording $\mathbf{5 j}^{7}$ (1.08 g, $\left.6.20 \mathrm{mmol}, 62 \%\right)$, as a colorless oil.

4-n-Butyl-1,2-dihydro-1-methylnaphthalene (11)<smiles>CCCCC1=CCC(C)c2ccccc21</smiles>

The reaction was performed as indicated for 1i. A mixture of 4-methyl-1-tetralone (1.42 g, $8.86 \mathrm{mmol})$ in $\mathrm{Et}_{2} \mathrm{O}(12.0 \mathrm{~mL})$ was added to a solution of $n$-BuMgI [prepared from 1-bromobutane (1,46 g, $10.6 \mathrm{mmol}), \mathrm{Mg}$ $(0.245 \mathrm{~g}, 10.1 \mathrm{mmol}), \mathrm{I}_{2}$ (some crystals) and anhydrous $\mathrm{Et}_{2} \mathrm{O}$ $(12.0 \mathrm{~mL})]$. The mixture was refluxed for $3 \mathrm{~h}$. The crude product was purified by flash column chromatography (gradient elution, 0-5\% of EtOAc in hexanes), affording the olefin $11^{2}(0.805 \mathrm{~g}, 4.02 \mathrm{mmol}, 45 \%)$, as a colorless oil. Starting material was recovered $(0.214 \mathrm{~g}, 1.34 \mathrm{mmol}, 15 \%)$.

\section{1,2-Dihydro-4-isopropyl-1-methylnaphthalene (1m)}<smiles>CC(C)C1=CCC(C)c2ccccc21</smiles>

The reaction was performed as indicated for 1i. A mixture of 4-methyl-1-tetralone $(0.961 \mathrm{~g}, 6.00 \mathrm{mmol})$ in $\mathrm{Et}_{2} \mathrm{O}(4.0 \mathrm{~mL}), i$-PrMgI [prepared from 2-bromopropane (1.93 g, $15.7 \mathrm{mmol}), \mathrm{Mg}\left(0.321 \mathrm{~g}, 13.2 \mathrm{mmol}\right.$ ), $\mathrm{I}_{2}$ (some crystals) in anhydrous $\left.\mathrm{Et}_{2} \mathrm{O}(6.0 \mathrm{~mL})\right]$ was stirred for $5.5 \mathrm{~h}$. The crude product was purified by flash column chromatography (gradient elution, $0-20 \%$ of EtOAc in hexanes), affording $\mathbf{1 m}^{8}(0.387 \mathrm{~g}, 2.08 \mathrm{mmol}, 35 \%)$ as a colorless oil.

\section{1,2-Dihydro-1-methyl-4-phenylnaphthalene (1n)}<smiles>CC1CC=C(c2ccccc2)c2ccccc21</smiles>

The reaction was performed as indicated for 1i. A mixture of 4-methyl-1-tetralone $(0.641 \mathrm{~g}, 4.00 \mathrm{mmol})$ in $\mathrm{Et}_{2} \mathrm{O}(0.5 \mathrm{~mL})$ and $\mathrm{PhMgBr}$ [prepared from bromobenzene 
(0.792 g, $5.04 \mathrm{mmol}), \mathrm{Mg}(0.117 \mathrm{~g}, 4.81 \mathrm{mmol}), \mathrm{I}_{2}$ (some crystals) in anhydrous $\left.\mathrm{Et}_{2} \mathrm{O}(1.0 \mathrm{~mL})\right]$ was refluxed for $1.5 \mathrm{~h}$. The crude product was purified by flash column chromatography (gradient elution, $10-15 \%$ of EtOAc in hexanes), affording the 1,2-dihydronaphthalene $\mathbf{1 n}^{9}$ (0.682 g, $3.10 \mathrm{mmol}, 78 \%)$, as a colorless oil.

4-(3,4-Dichlorophenyl)1,2-dihydro-1-methylnaphthalene (1o)<smiles>CC1CC=C(c2ccc(Cl)c(Cl)c2)c2ccccc21</smiles>

The reaction was performed as indicated for 1i. A mixture of 4-methyl-1-tetralone $(0.645 \mathrm{~g}, 4.03 \mathrm{mmol})$ in $\mathrm{Et}_{2} \mathrm{O}(0.5 \mathrm{~mL})$ and $1,2-\mathrm{ClPhMgBr}$ [prepared from 4-bromo1,2-dichlorobenzene $(1.15 \mathrm{~g}, 5.09 \mathrm{mmol}), \mathrm{Mg}(0.117 \mathrm{~g}$, $4.81 \mathrm{mmol}), \mathrm{I}_{2}$ (some crystals) in anhydrous $\mathrm{Et}_{2} \mathrm{O}(1.0 \mathrm{~mL})$ ] was refluxed for $2 \mathrm{~h}$. The crude product was purified by flash column chromatography (gradient elution, $10-30 \%$ of EtOAc in hexanes), affording the 1-(3,4-dichloropheny)1,2,3,4-tetrahydro-4-methylnaphthalen-1-ol (0.870 g, $2.83 \mathrm{mmol}, 70 \%)$, as a colorless oil. The isolated alcohol was dissolved in anhydrous toluene $(3.5 \mathrm{~mL})$. Some crystals of $p$-toluenesulfonic acid were added to that solution. The reaction was refluxed for $6 \mathrm{~h}$. The reaction was extracted with EtOAc. The organic phase was washed with $\mathrm{H}_{2} \mathrm{O}$, saturated solution of $\mathrm{NaHCO}_{3}$, saturated solution of $\mathrm{NaCl}$ and dried over anhydrous $\mathrm{MgSO}_{4}$. The crude product was purified by flash column chromatography (isocratic elution with hexanes), furnishing the desired alkene 10 $(0.385 \mathrm{~g}, 1.33 \mathrm{mmol}, 56 \%)$, as a colorless oil; IR $v_{\max } / \mathrm{cm}^{-1}$ (film) $1121,1258,1515,2830,2934 ;{ }^{1} \mathrm{H}$ NMR $(300 \mathrm{MHz}$, $\left.\mathrm{CDCl}_{3}\right) \delta 1.30(\mathrm{~d}, 1 \mathrm{H}, J$ 7,0 Hz), $2.22(\mathrm{ddd}, 1 \mathrm{H}, J 16.8$, 7.7 and $5.0 \mathrm{~Hz}$ ), 2.55 (ddd, $1 \mathrm{H}, J 16.8,6.5$ and $4.4 \mathrm{~Hz}$ ), 2.98 (sext, 1H, J 7.0 Hz), 6.00 (t, 1H, J 4.7 Hz), 6.93-6.96 $(\mathrm{m}, 1 \mathrm{H}), 7.10-7.26(\mathrm{~m}, 4 \mathrm{H}), 7.42-7.46(\mathrm{~m}, 1 \mathrm{H}) ;{ }^{13} \mathrm{C} \mathrm{NMR}$ $\left(75 \mathrm{MHz}, \mathrm{CDCl}_{3}\right) \delta 19.8,31.4,32.1,125.3,126.2,126.2$, $127.4,127.7,128.1,130.2,130.5,131.0,132.3,133.4$, 137.5, 140.9, 141.5; HRMS $(\mathrm{m} / \mathrm{z})$ calcd. for $\mathrm{C}_{14} \mathrm{H}_{20} \mathrm{O}_{4}$ $[\mathrm{M}+\mathrm{Na}]^{+} 275.1254$, found 275.1252 .

\section{6,7-Dihydro-9-methyl-5H-benzo[7]annulene (1p)}<smiles>CC1=CCCCc2ccccc21</smiles>

The reaction was performed as indicated for 1i. A mixture of 1-benzosuberone $(0.481 \mathrm{~g}, 3.00 \mathrm{mmol})$ in anhydrous $\mathrm{Et}_{2} \mathrm{O}(2.0 \mathrm{~mL}), \mathrm{MeMgI}$ [prepared from MeI $(0.5 \mathrm{~mL}, 8.10 \mathrm{mmol}), \mathrm{Mg}(0.202 \mathrm{~g}, 8.31 \mathrm{mmol})$ and $\mathrm{I}_{2}$ (some crystals) in anhydrous $\left.\mathrm{Et}_{2} \mathrm{O}(2.0 \mathrm{~mL})\right]$ was stirred for $4 \mathrm{~h}$ under reflux. The crude product was purified by flash column chromatography (gradient elution, $0-10 \%$ of EtOAc in hexanes), affording $\mathbf{1 p}^{10}(0.403 \mathrm{~g}, 2.55 \mathrm{mmol}$, $85 \%$ ), as a colorless oil.

\section{References}

1. Ferraz, H. M. C.; Carneiro, V. M. T.; Silva Jr., L. F.; Synthesis 2009, 385 (see ref. 13 in the article).

2. Ferraz, H. M. C.; Silva Jr., L. F.; Vieira, T. O.; Tetrahedron 2001, 57, 1709 (see ref. 9 in the article).

3. Silva Jr., L. F.; Sousa, R. M. F.; Ferraz, H. M. C.; Aguilar, A. M.; J. Braz. Chem. Soc. 2005, 16, 1160 (see ref. 8 in the article).

4. Silva Jr., L. F.; Siqueira, F. A.; Pedrozo, E. C.; Vieira, F. Y. M.; Doriguetto, A. C.; Org. Lett. 2007, 9, 1433 (see ref. 7 in the article).

5. Allinger, N. L.; Jones, E. S.; J. Org. Chem. 1962, 2770.

6. Kato, K.; Terauchi, J.; Mori, M.; Suzuki, N.; Shimomura, Y.; Takekawa, S.; Ishihara, Y.; PCT Int. Appl. WO 2001021577 2001, 363.

7. Radcliffe, M. M.; Weber, W. P.; J. Org. Chem. 1977, 42, 297.

8. Bhonsle, J. B.; Indian J. Chem. B 1995, 34B, 372.

9. Newman, M. S.; Anderson, H. V.; Takemura, K. H.; J. Am. Chem. Soc. 1953, 75, 347.

10. Russel, M. G. N.; Baker, R.; Billington, D. C.; Knight, A. K.; Middlemiss, D. N.; Noble, A. J.; J. Med. Chem. 1992, 35, 2025. 


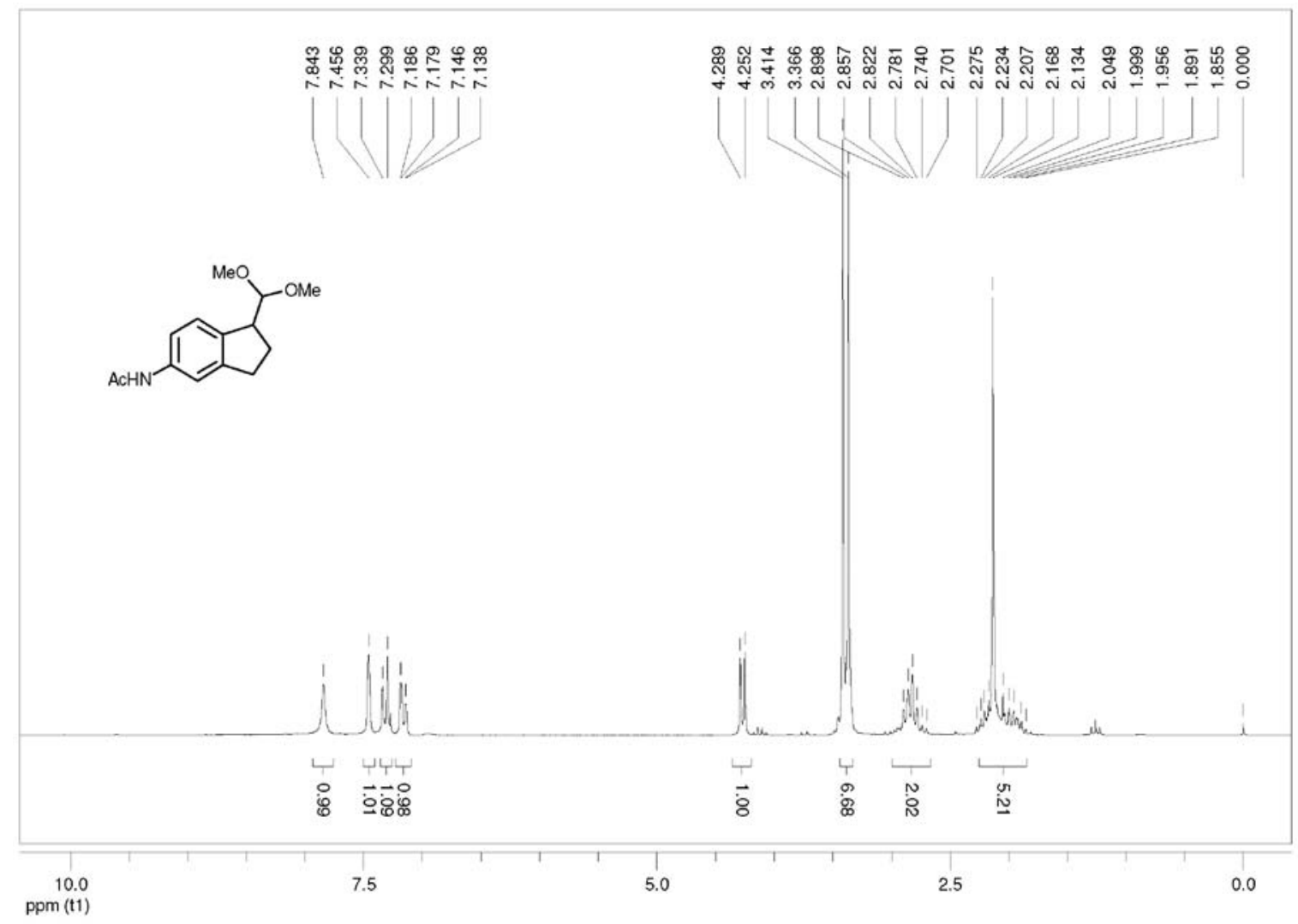

Figure S1. ${ }^{1} \mathrm{H}$ NMR spectrum of $\mathbf{2 d}\left(\mathrm{CDCl}_{3}\right.$, TMS, $\left.200 \mathrm{MHz}, \delta\right)$.

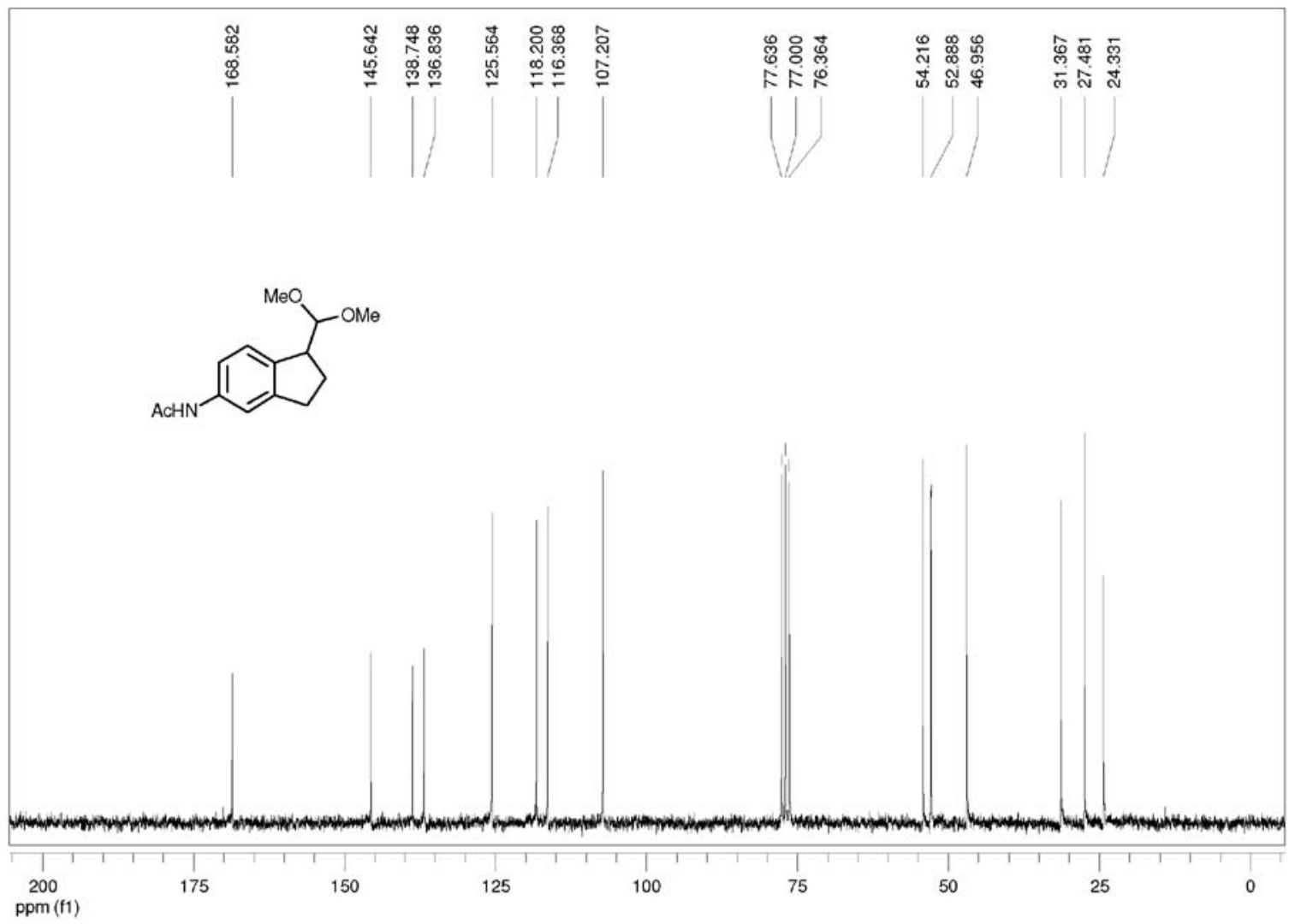

Figure S2. ${ }^{13} \mathrm{C}$ NMR spectrum of $\mathbf{2 d}\left(\mathrm{CDCl}_{3}, \mathrm{TMS}, 50 \mathrm{MHz}, \delta\right)$. 


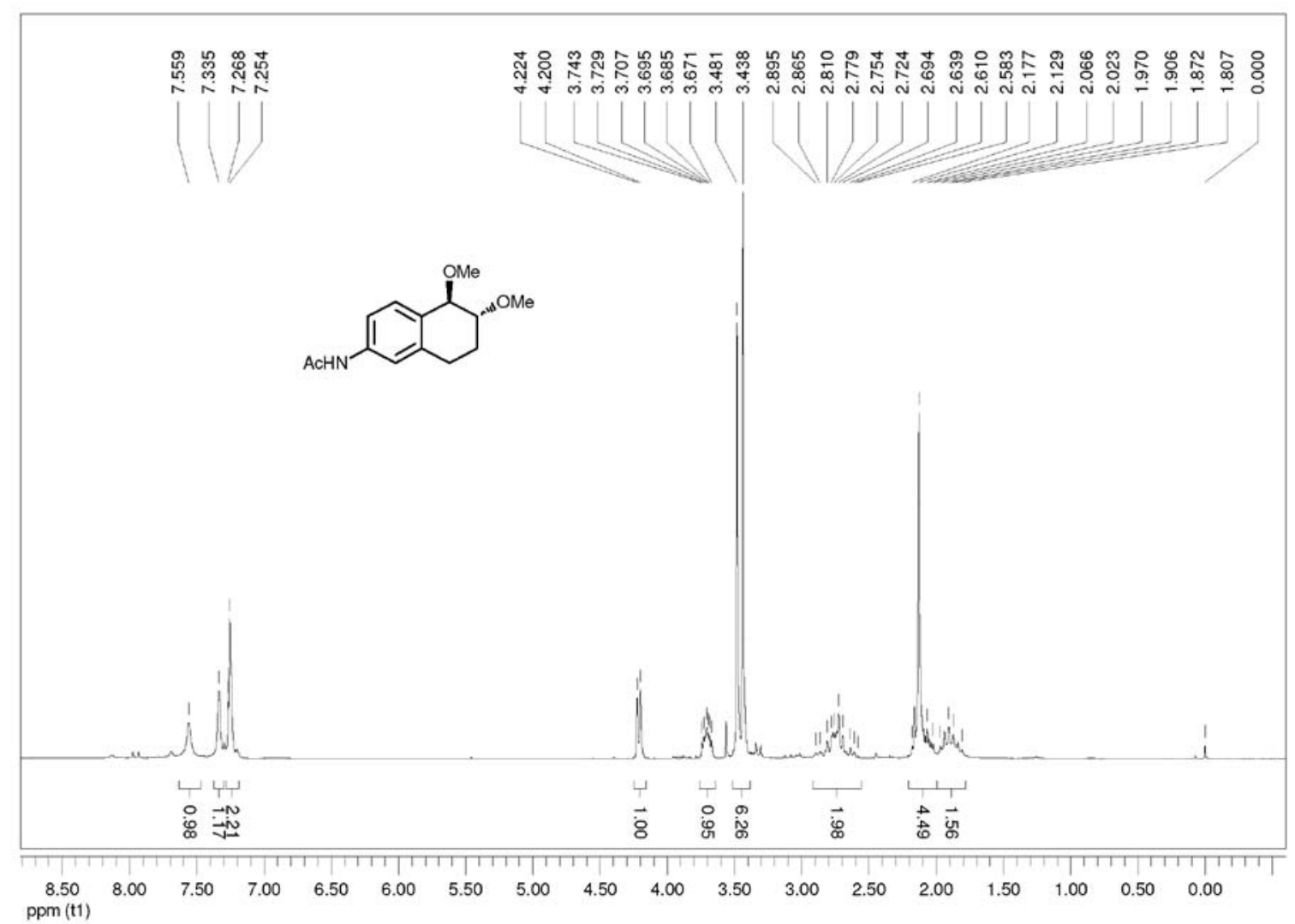

Figure S3. ${ }^{1} \mathrm{H}$ NMR spectrum of trans-3d $\left(\mathrm{CDCl}_{3}\right.$, TMS, $\left.200 \mathrm{MHz}, \delta\right)$.

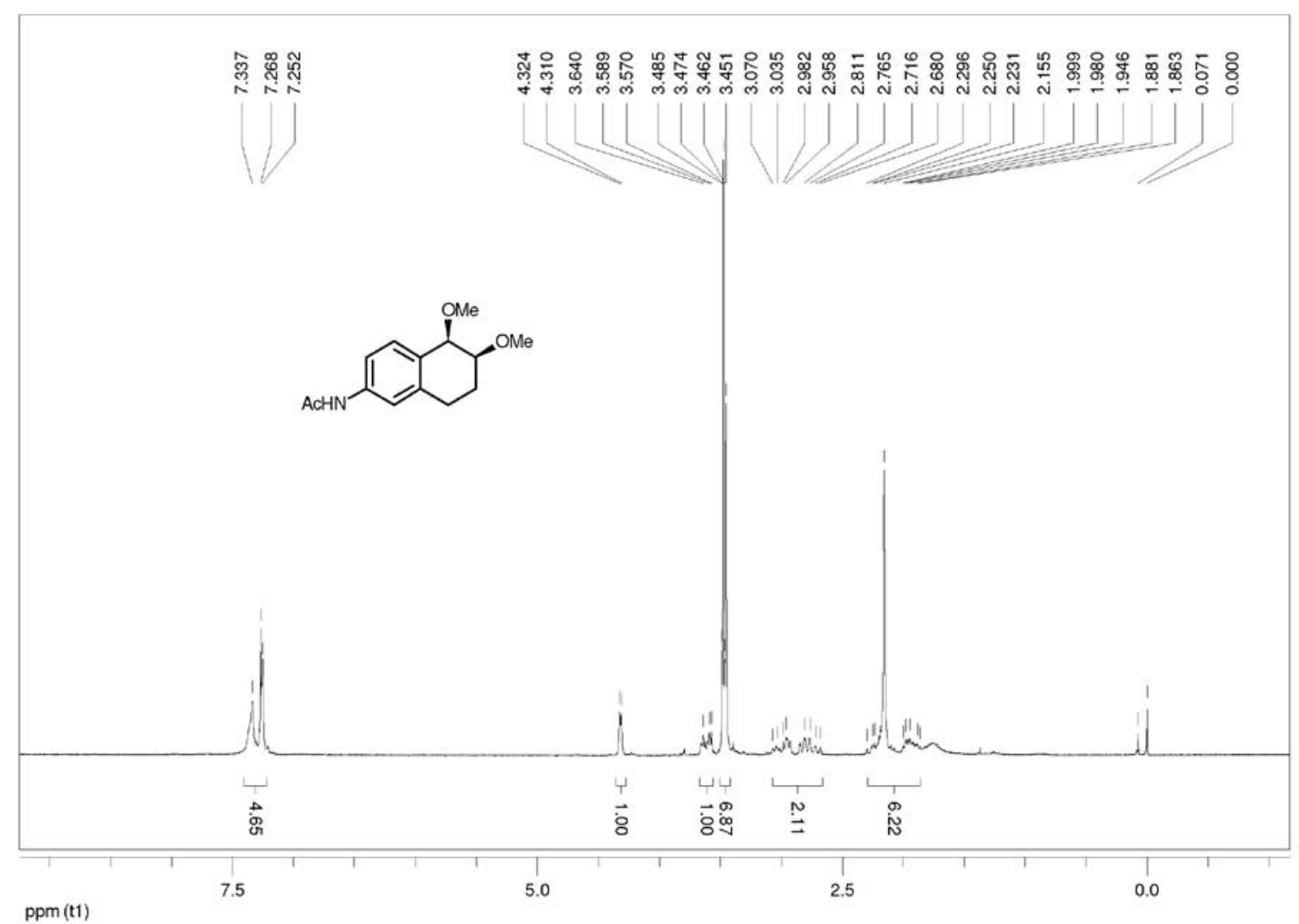

Figure S4. ${ }^{1} \mathrm{H}$ NMR spectrum of $c i s-3 d\left(\mathrm{CDCl}_{3}\right.$, TMS, $\left.200 \mathrm{MHz}, \delta\right)$. 


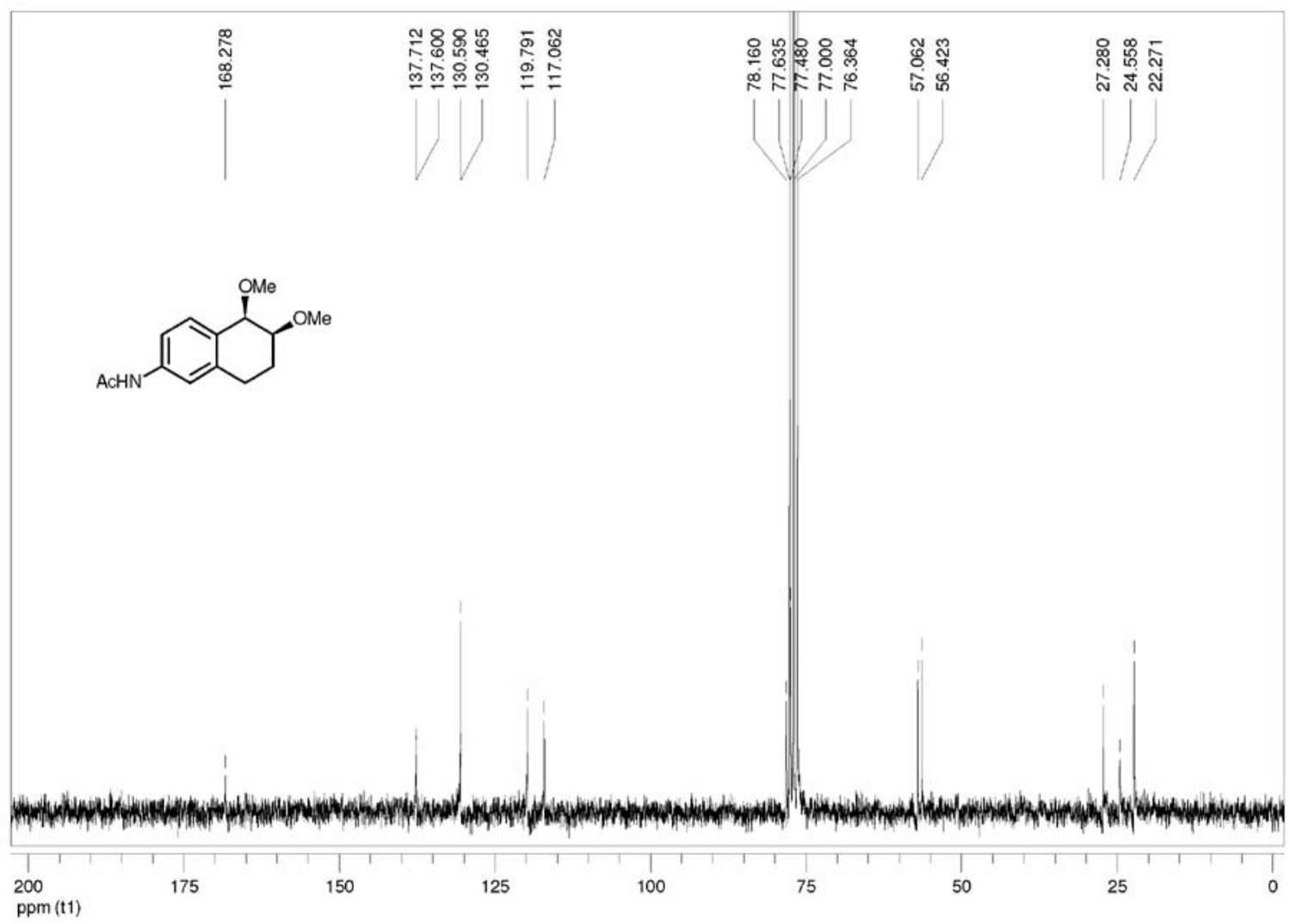

Figure S5. ${ }^{13} \mathrm{C}$ NMR spectrum of $c i s-3 d\left(\mathrm{CDCl}_{3}, \mathrm{TMS}, 75 \mathrm{MHz}, \delta\right)$.

m

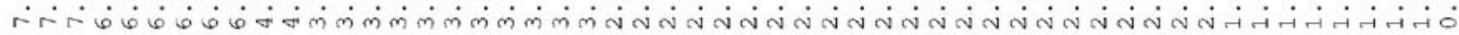

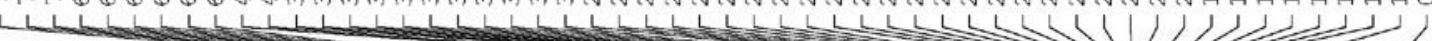<smiles>COc1ccc2c(c1)[C@@H](OC)[C@H](OC)CC2</smiles>
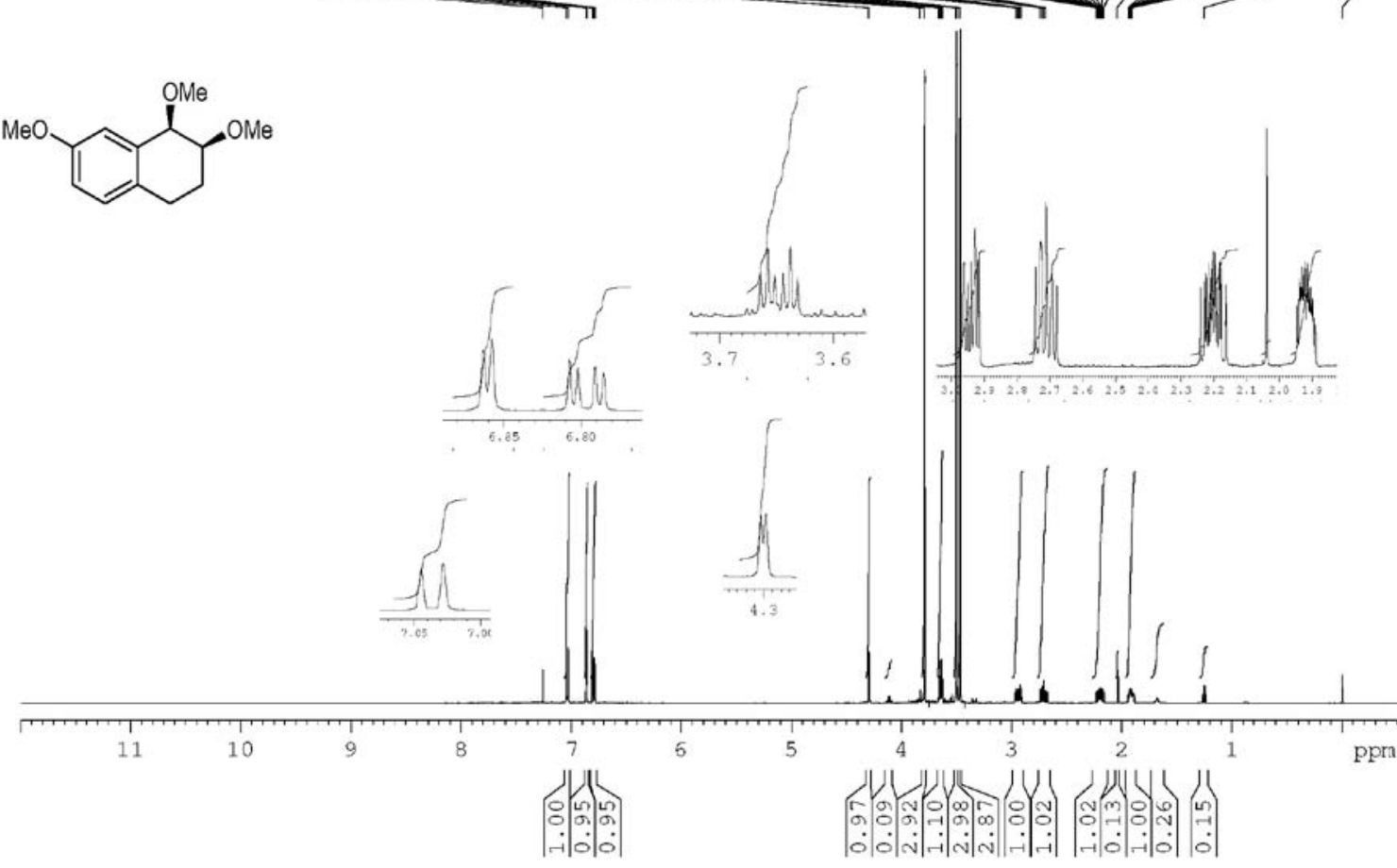

Figure S6. ${ }^{~} \mathrm{H}$ NMR spectrum of $c i s-3 f\left(\mathrm{CDCl}_{3}\right.$, TMS, $\left.500 \mathrm{MHz}, \delta\right)$. 


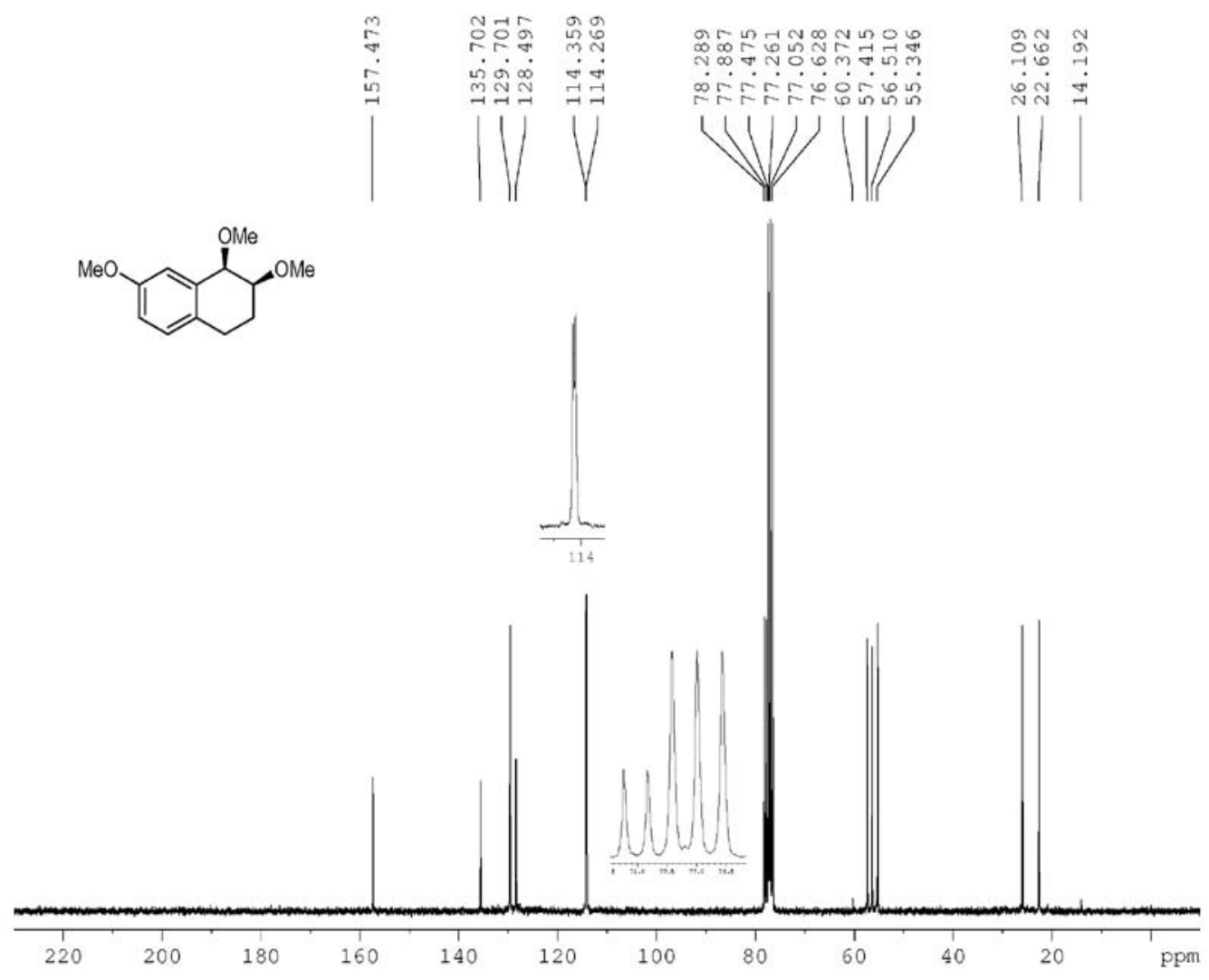

Figure S7. ${ }^{13} \mathrm{C}$ NMR spectrum of $c i s-3 \mathbf{f}\left(\mathrm{CDCl}_{3}\right.$, TMS, $\left.75 \mathrm{MHz}, \delta\right)$.

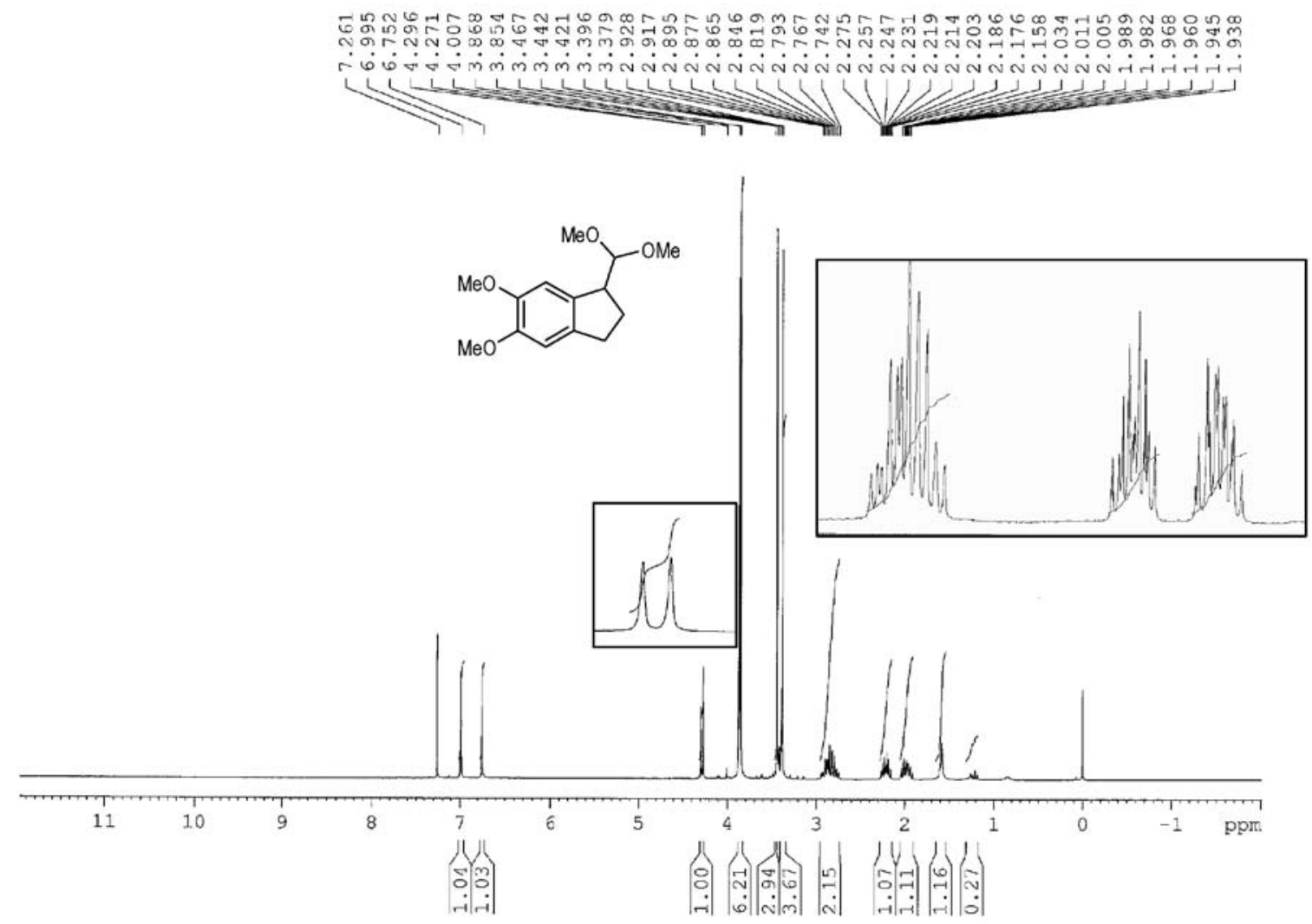

Figure S8. ${ }^{1} \mathrm{H}$ NMR spectrum of $\mathbf{2 g}\left(\mathrm{CDCl}_{3}, \mathrm{TMS}, 300 \mathrm{MHz}, \delta\right)$. 

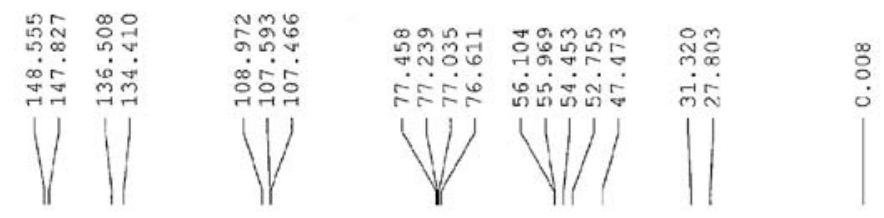<smiles>COc1cc2c(cc1OC)C(C(OC)OC)CC2</smiles>
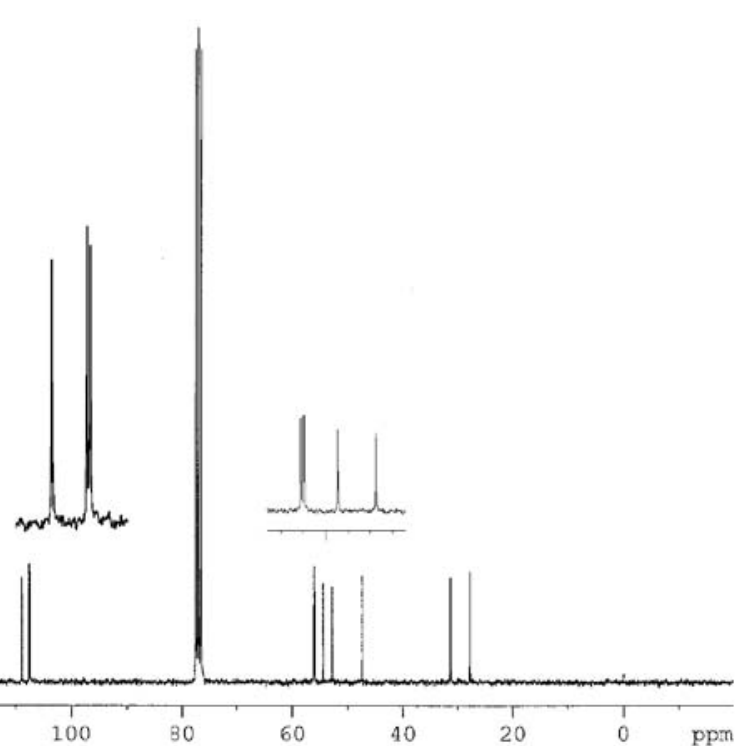

Figure S9. ${ }^{13} \mathrm{C}$ NMR spectrum of $\mathbf{2 g}\left(\mathrm{CDCl}_{3}, \mathrm{TMS}, 75 \mathrm{MHz}, \delta\right)$.
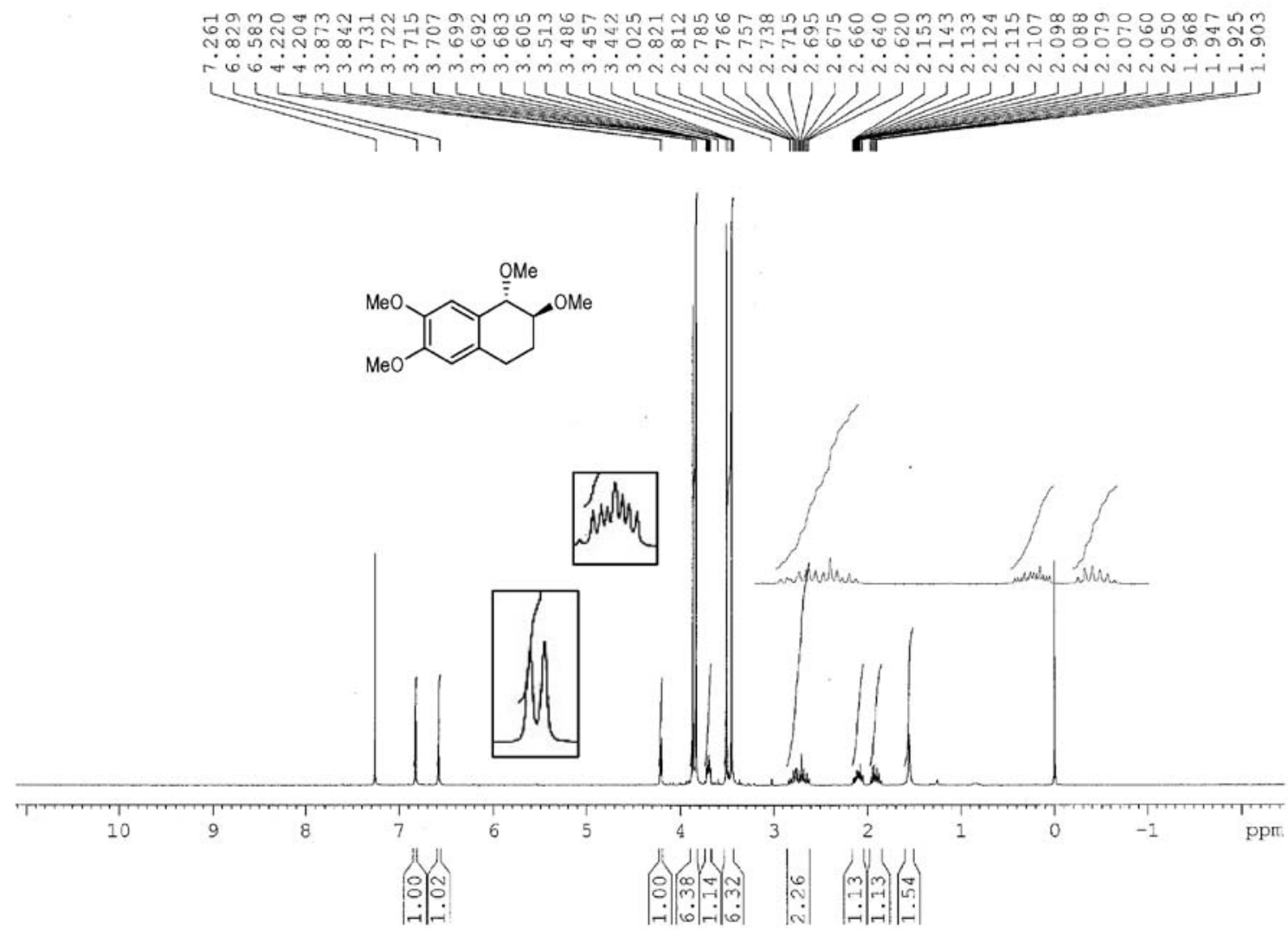

Figure S10. ${ }^{1} \mathrm{H}$ NMR spectrum of trans-3g $\left(\mathrm{CDCl}_{3}, \mathrm{TMS}, 300 \mathrm{MHz}, \delta\right)$. 

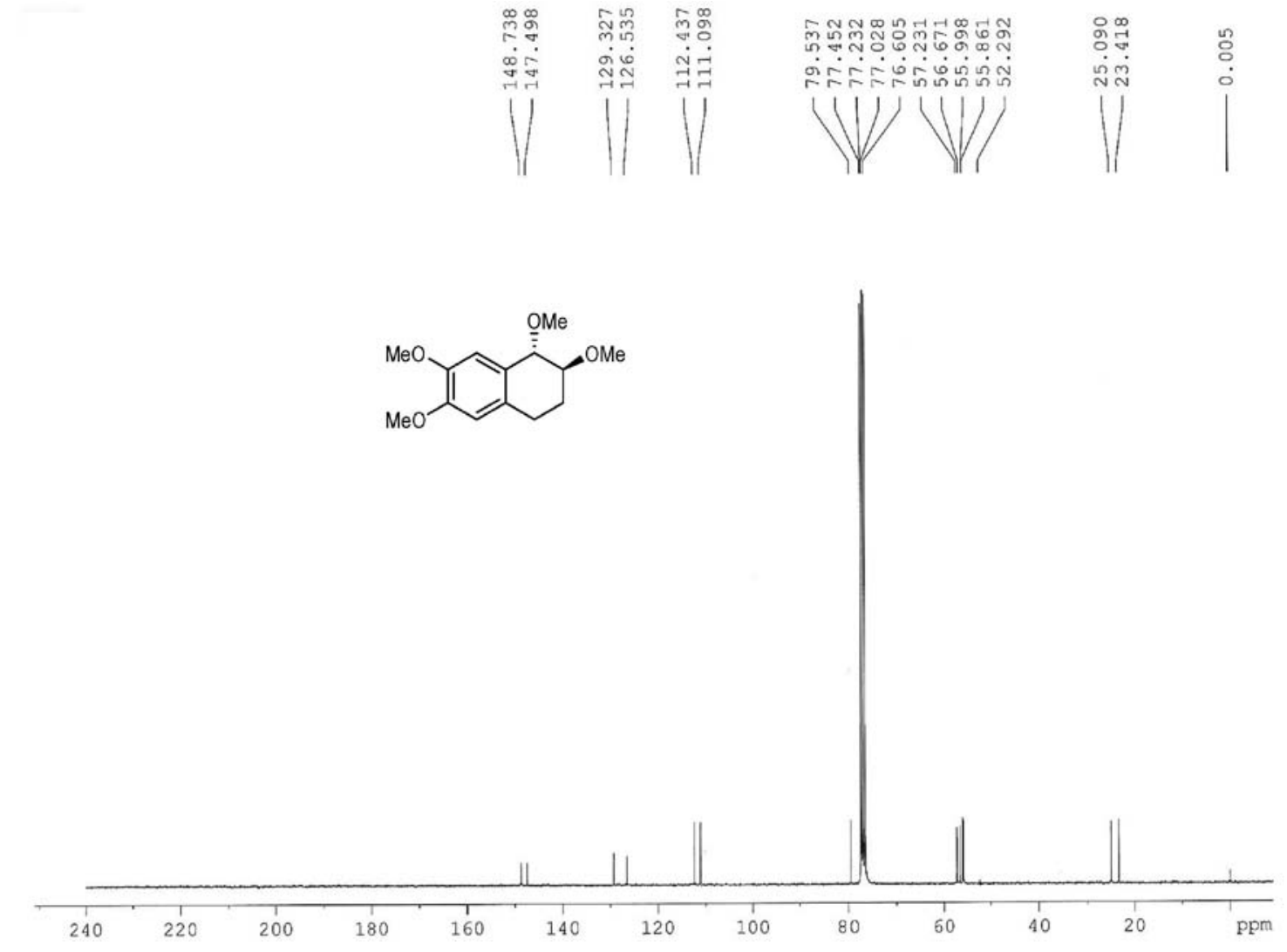

Figure S11. ${ }^{13} \mathrm{C}$ NMR spectrum of trans-3g $\left(\mathrm{CDCl}_{3}, \mathrm{TMS}, 75 \mathrm{MHz}, \delta\right)$.

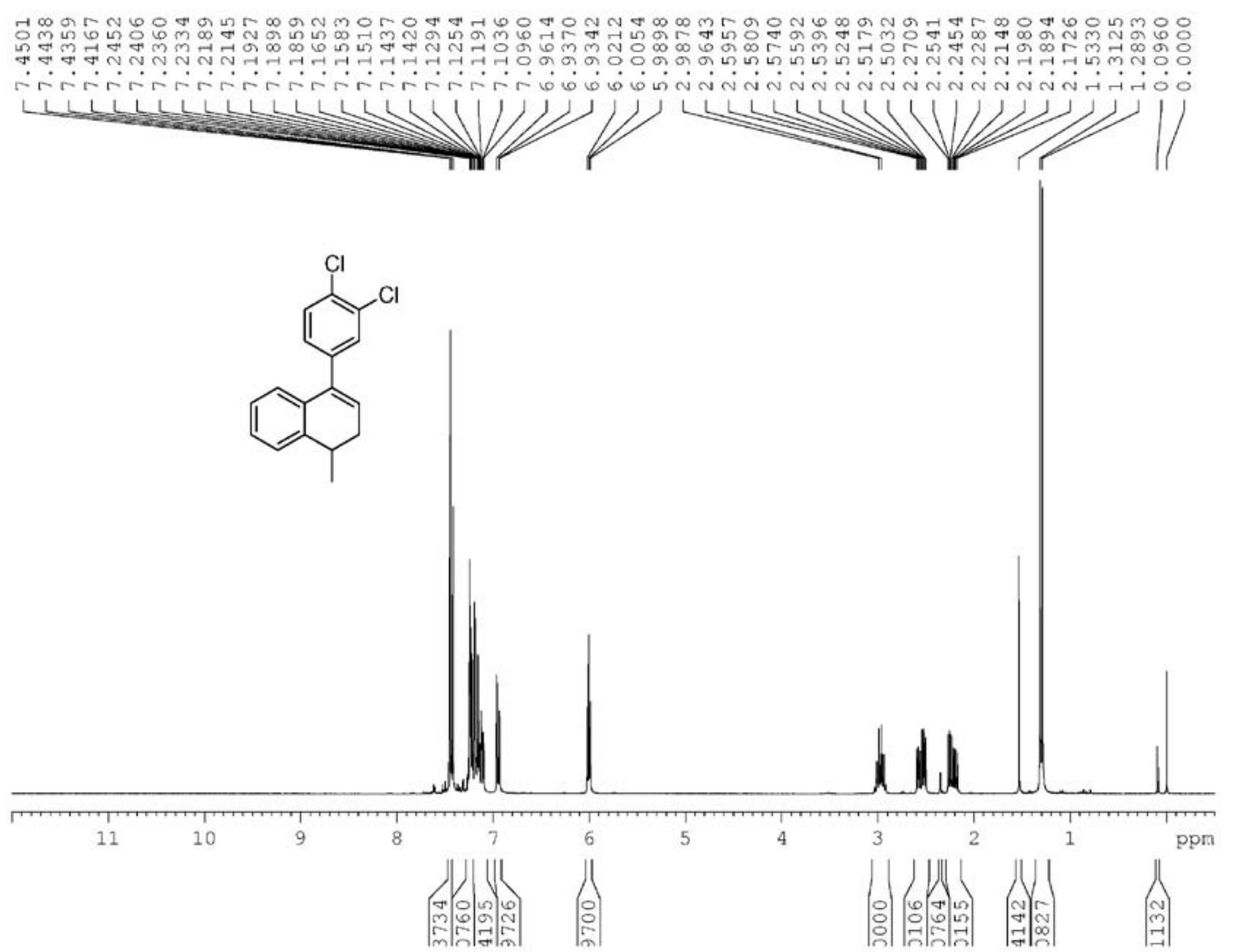

Figure S12. ${ }^{1} \mathrm{H}$ NMR spectrum of $10\left(\mathrm{CDCl}_{3}\right.$, TMS, $\left.300 \mathrm{MHz}, \delta\right)$. 

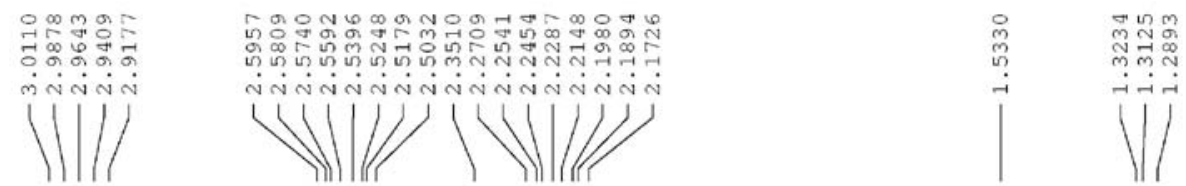<smiles>CC1CC=C(c2ccc(Cl)c(Cl)c2)c2ccccc21</smiles>

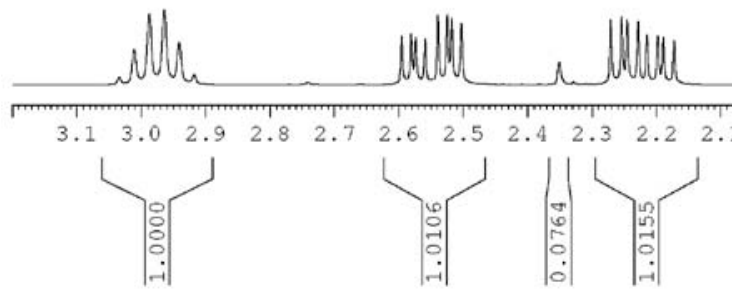

Figure S13. ${ }^{1} \mathrm{H}$ NMR spectrum of $\mathbf{1 0}\left(\mathrm{CDCl}_{3}\right.$, TMS, $\left.300 \mathrm{MHz}, \delta\right)$ - expansion.

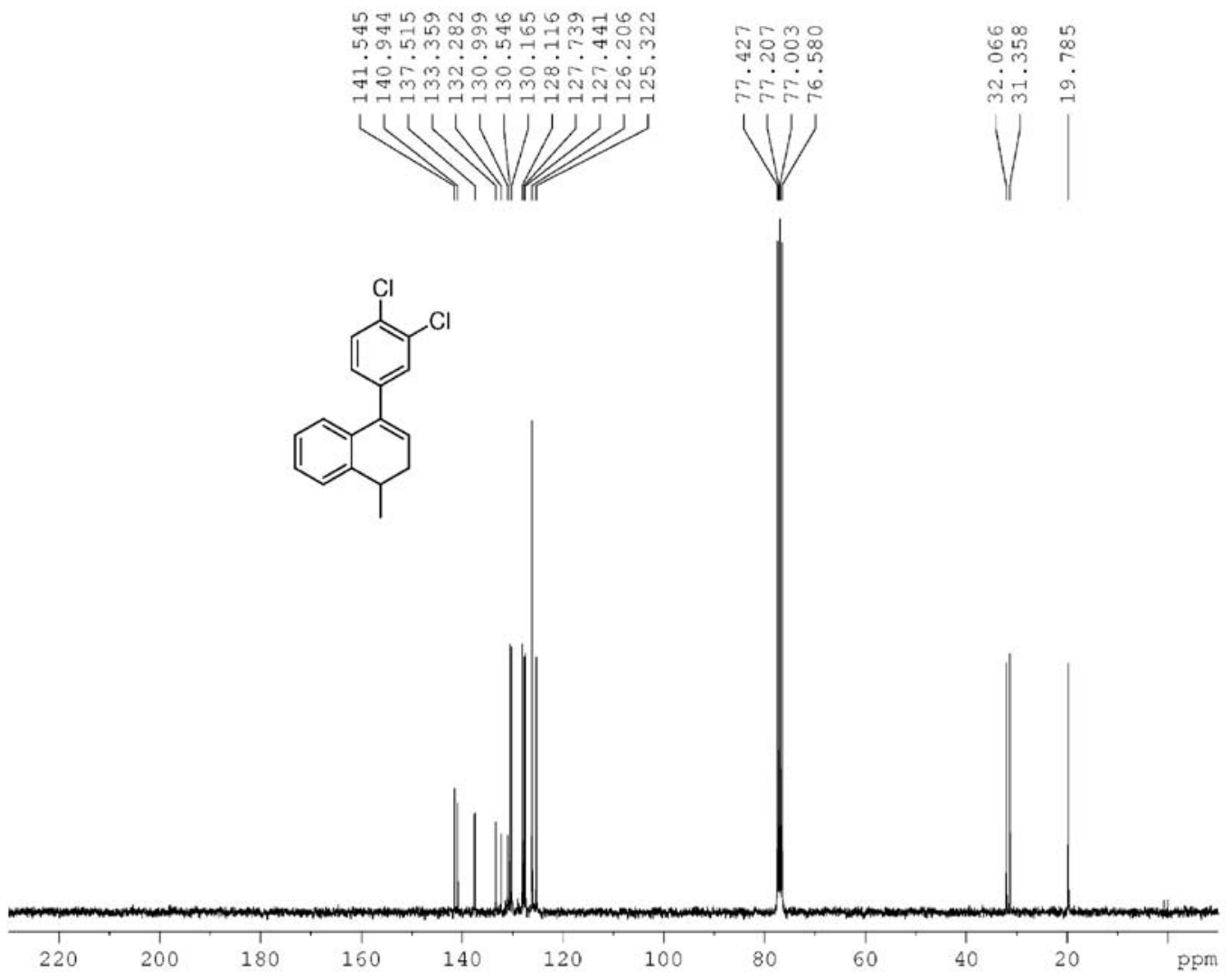

Figure S14. ${ }^{13} \mathrm{C}$ NMR spectrum of $10\left(\mathrm{CDCl}_{3}\right.$, TMS, $\left.75 \mathrm{MHz}, \delta\right)$. 

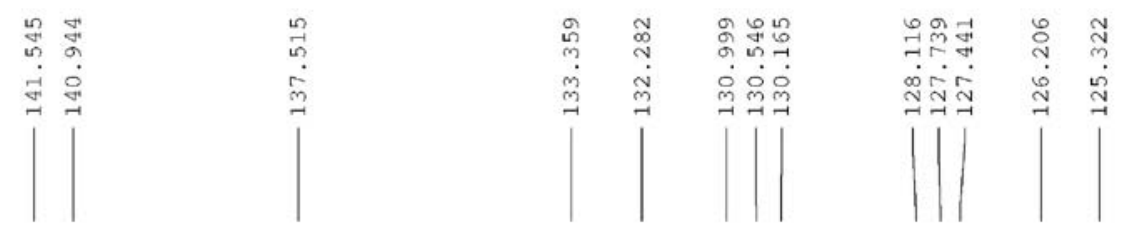<smiles>CC1CC=C(c2ccc(Cl)c(Cl)c2)c2ccccc21</smiles>

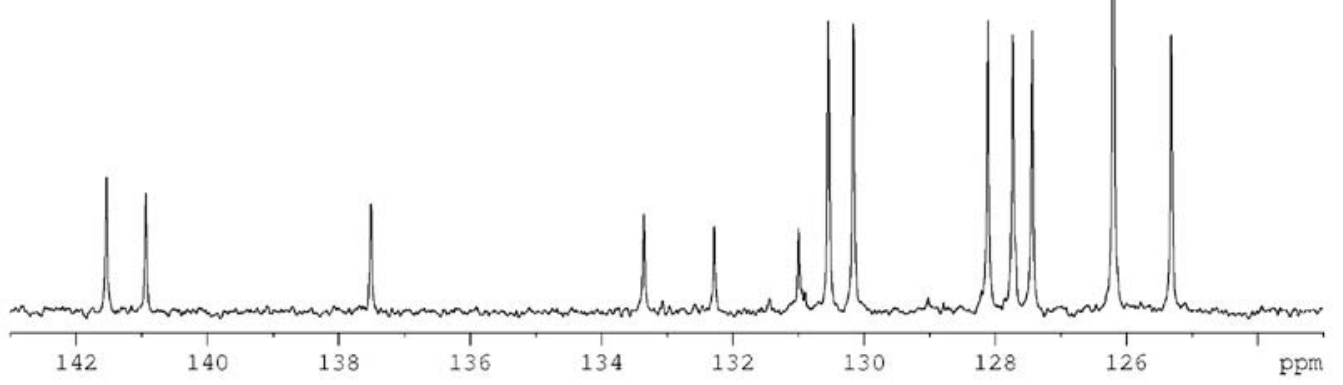

Figure S15. ${ }^{13} \mathrm{C}$ NMR spectrum of $10\left(\mathrm{CDCl}_{3}, \mathrm{TMS}, 75 \mathrm{MHz}, \delta\right)$ - expansion.

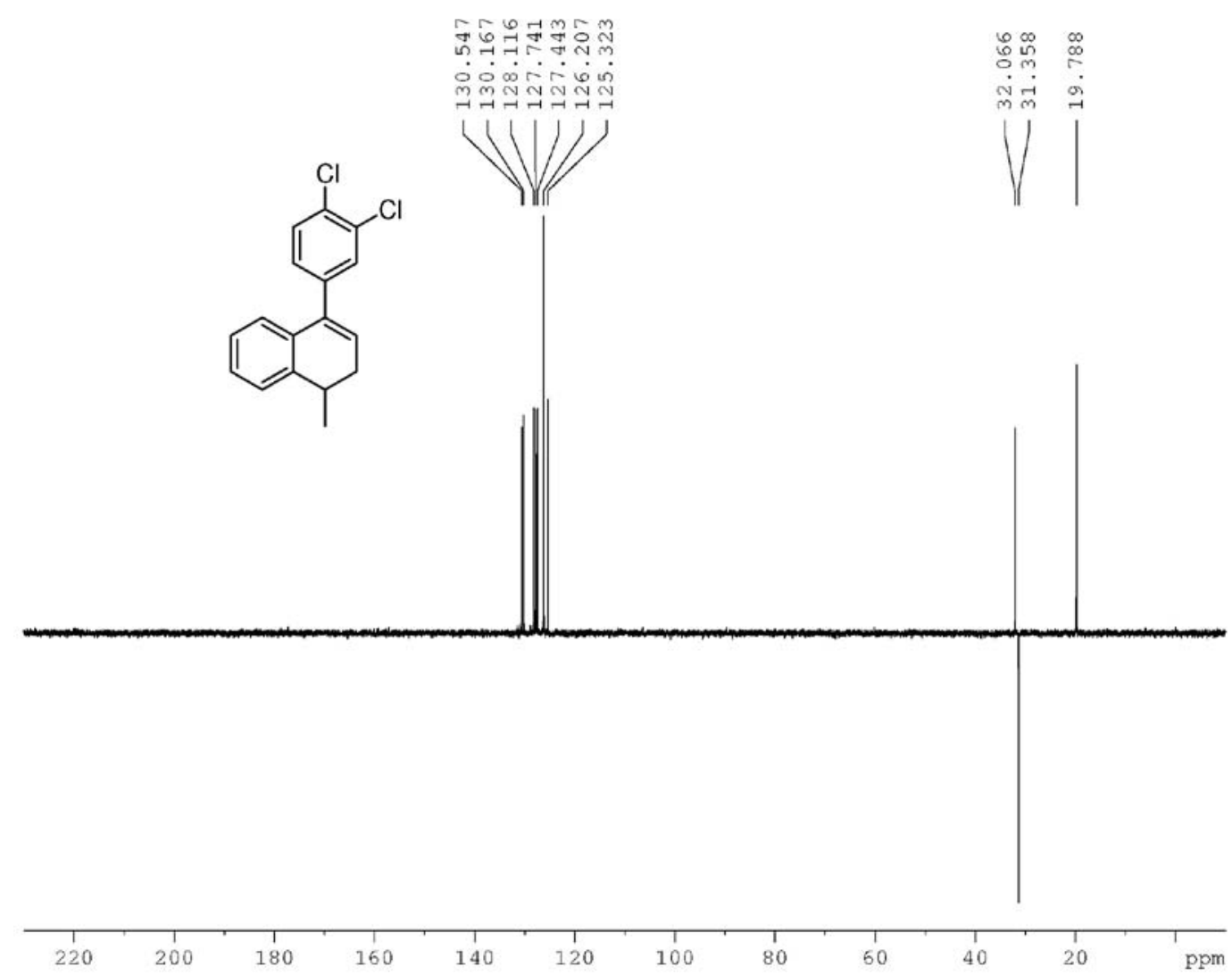

Figure S16. DEPT 135 spectrum of $\mathbf{1 o}\left(\mathrm{CDCl}_{3}, \mathrm{TMS}, 75 \mathrm{MHz}, \delta\right)$. 


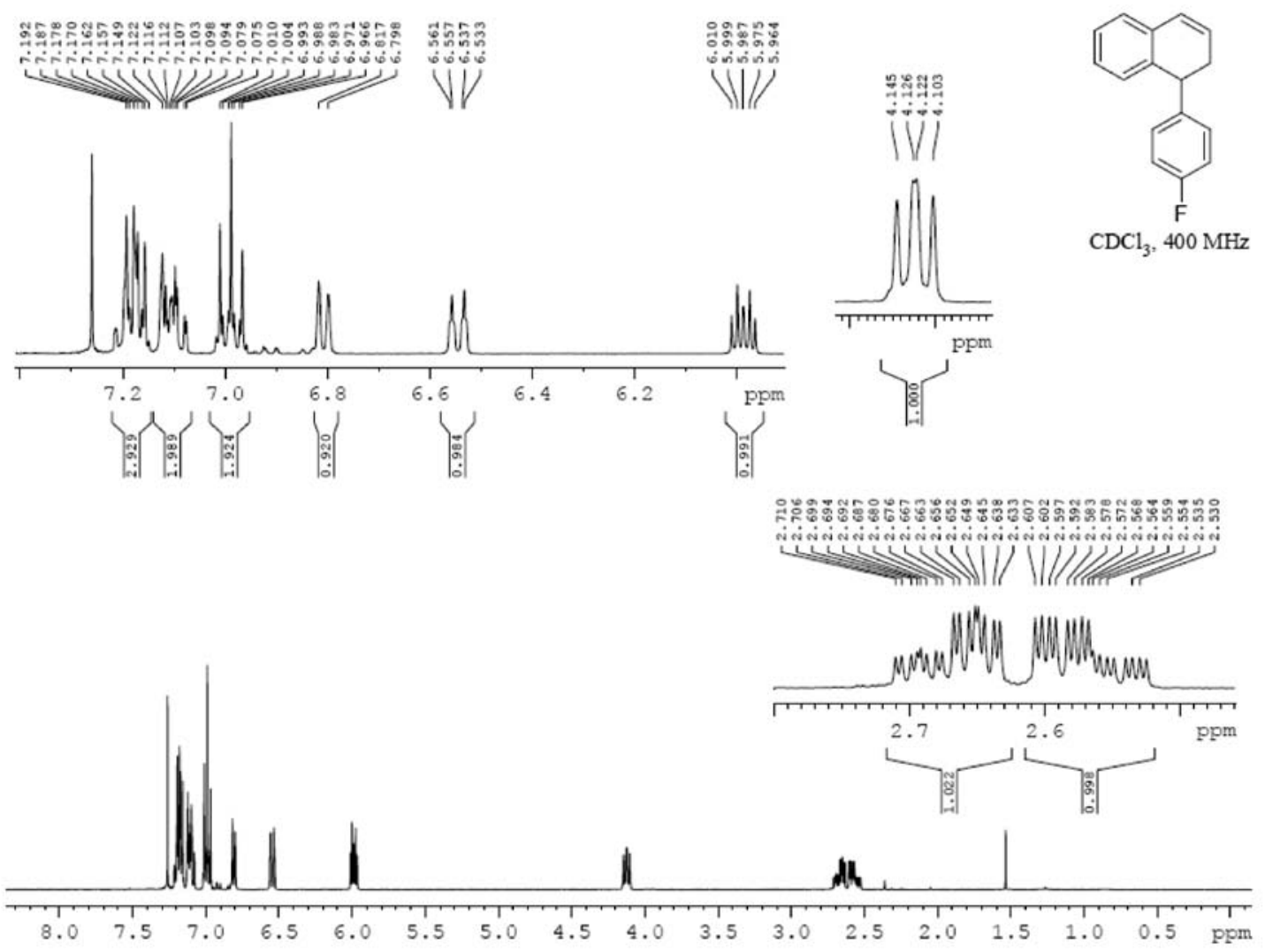

Figure S17. ${ }^{1} \mathrm{H}$ NMR spectrum of $\mathbf{1 q}\left(\mathrm{CDCl}_{3}\right.$, TMS, $\left.400 \mathrm{MHz}, \delta\right)$.

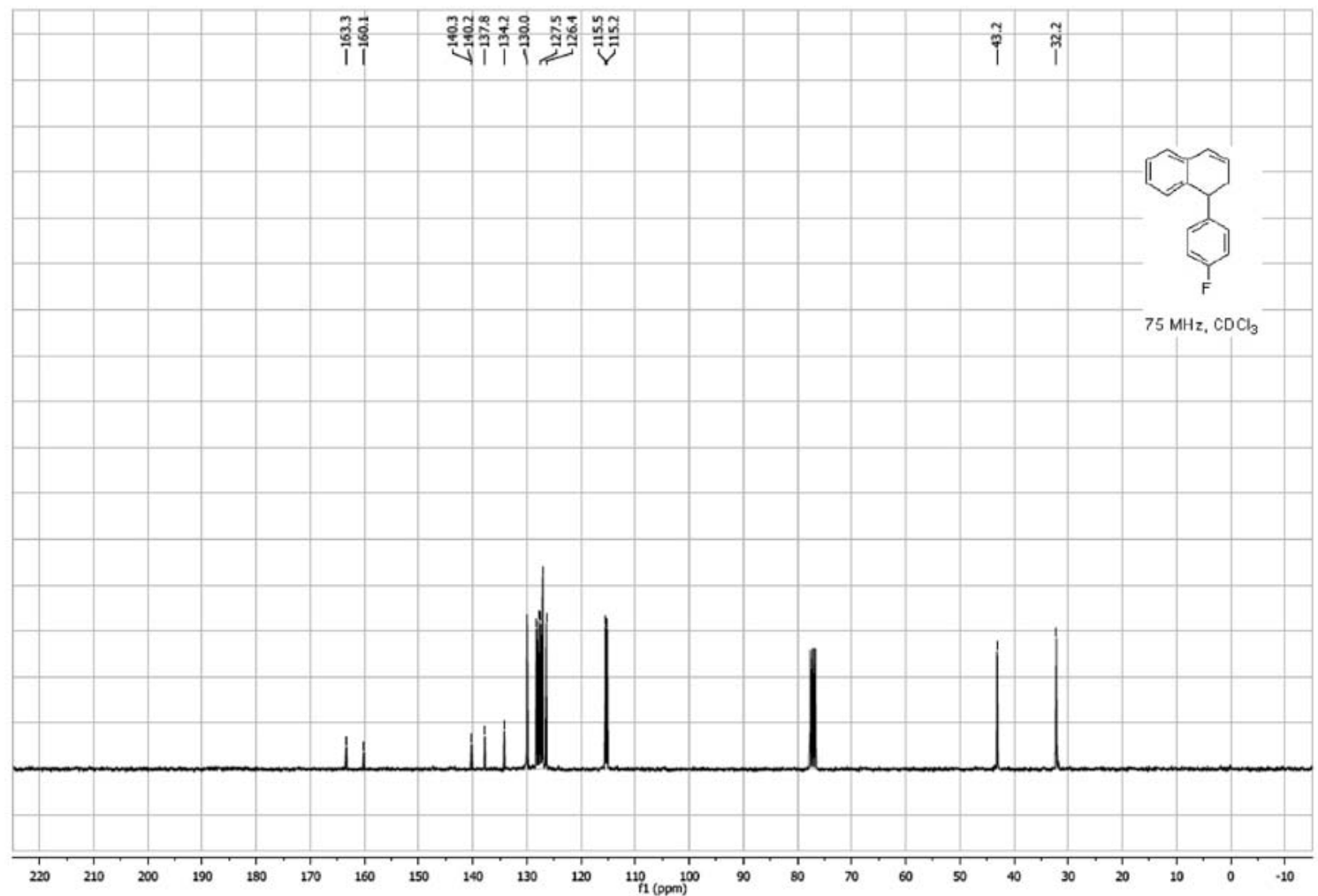

Figure S18. ${ }^{13} \mathrm{C}$ NMR spectrum of $\mathbf{1 q}\left(\mathrm{CDCl}_{3}, \mathrm{TMS}, 75 \mathrm{MHz}, \delta\right)$. 


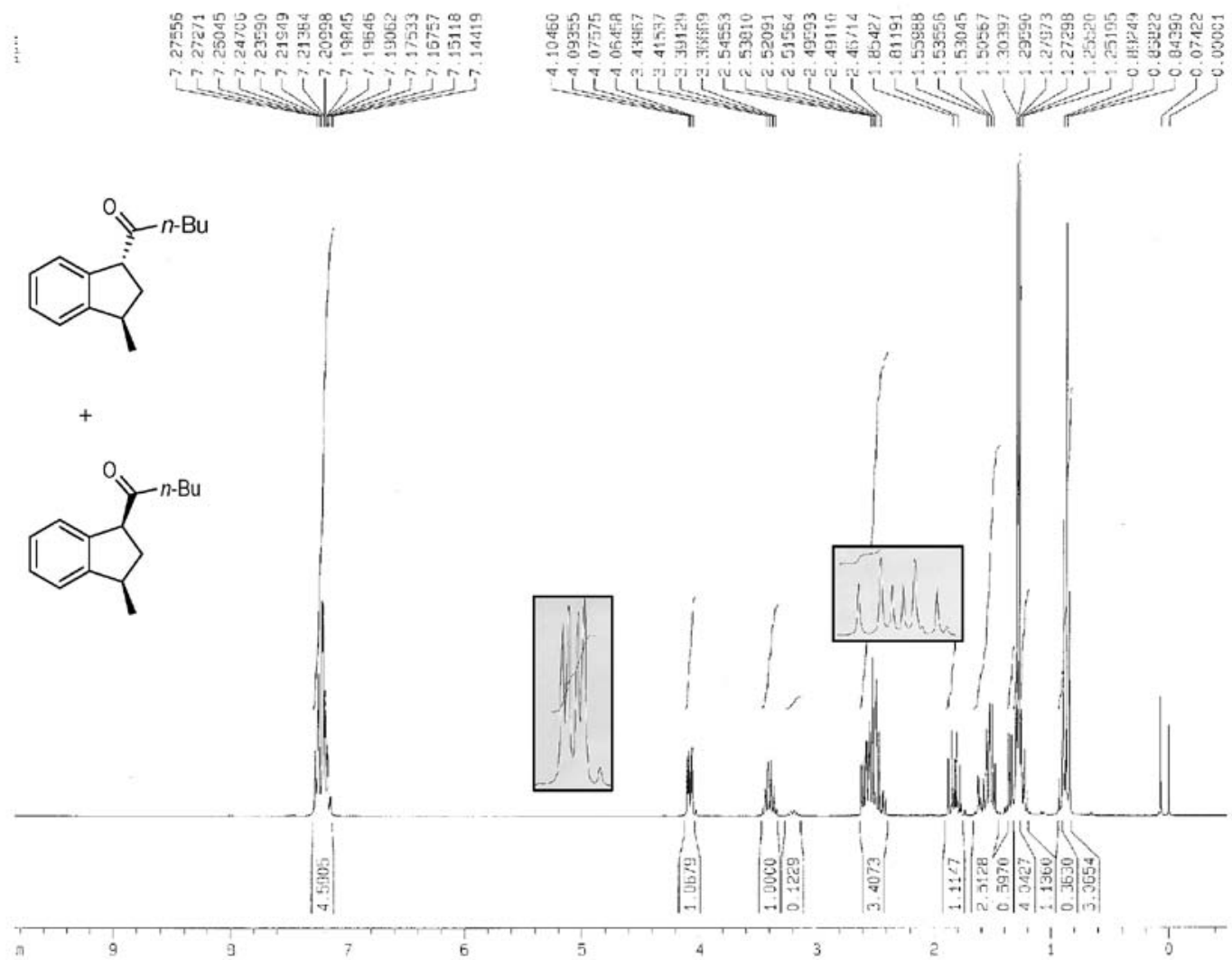

Figure S19. ${ }^{1} \mathrm{H}$ NMR spectrum of $\mathbf{5 l}\left(\mathrm{CDCl}_{3}\right.$, TMS, $\left.300 \mathrm{MHz}, \delta\right)$.

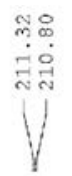

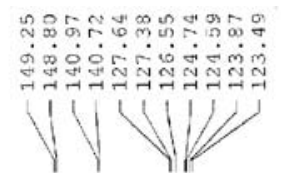

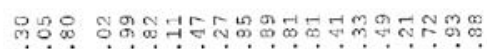

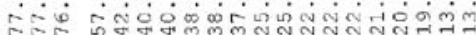
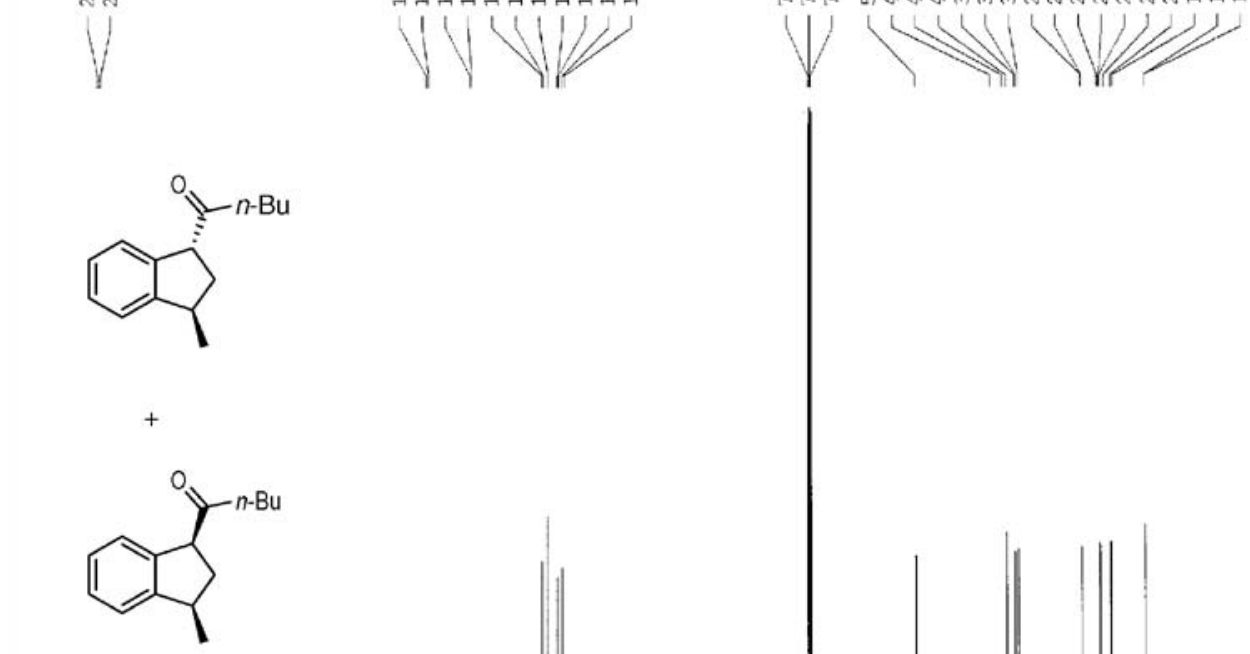<smiles>CCCCC(=O)[C@H]1C[C@H](C)c2ccccc21</smiles>

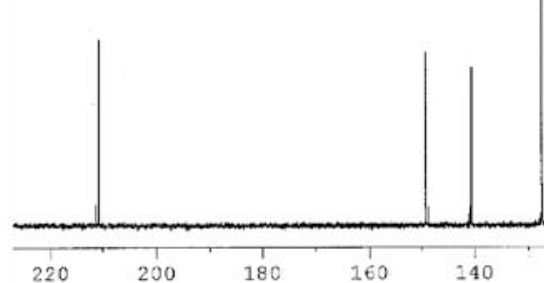

$200-180$

Figure S20. ${ }^{13} \mathrm{C}$ NMR spectrum of $\mathbf{5 l}\left(\mathrm{CDCl}_{3}, \mathrm{TMS}, 75 \mathrm{MHz}, \delta\right)$. 


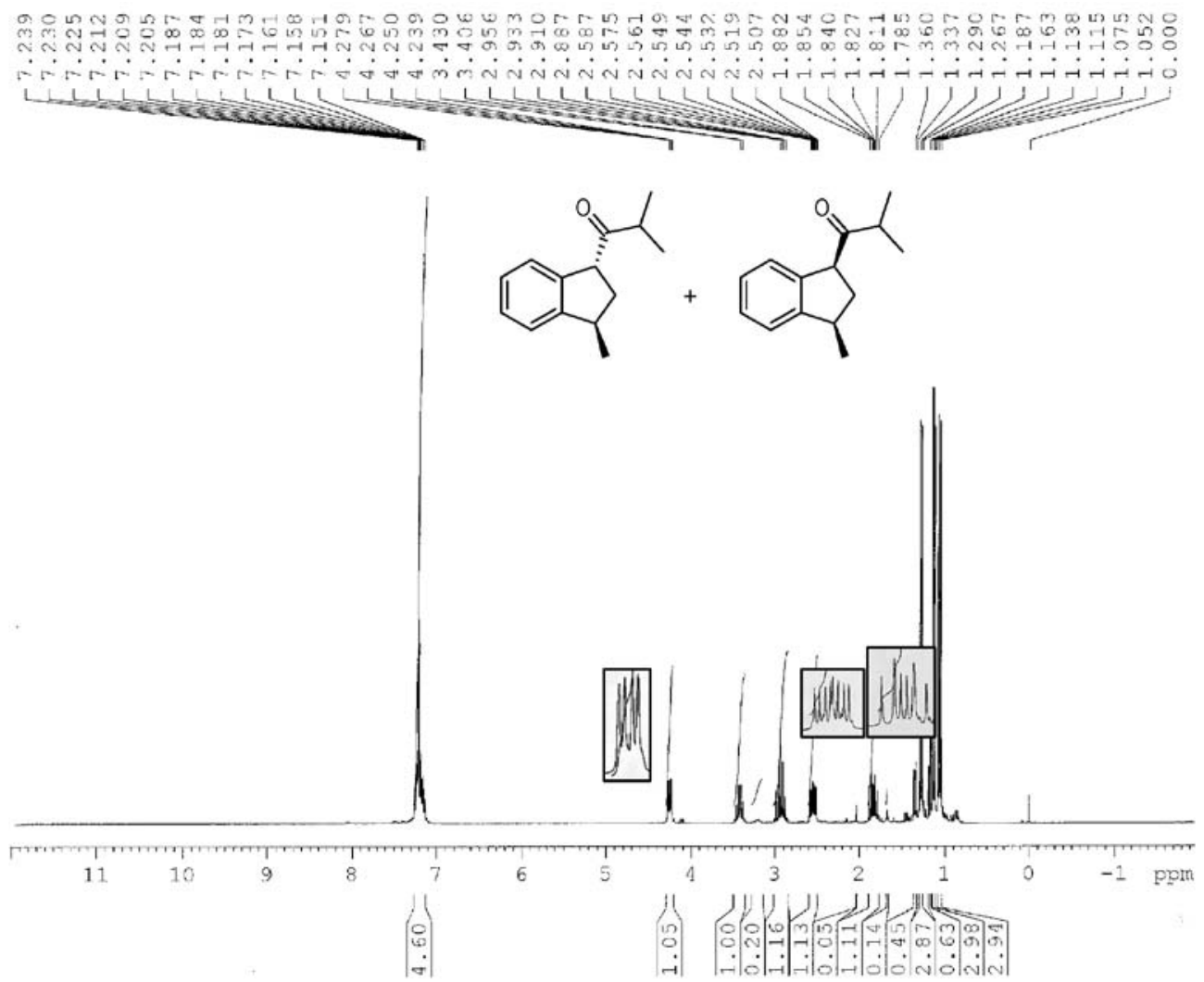

Figure S21. ${ }^{1} \mathrm{H}$ NMR spectrum of $\mathbf{5 m}\left(\mathrm{CDCl}_{3}\right.$, TMS, $\left.300 \mathrm{MHz}, \delta\right)$.

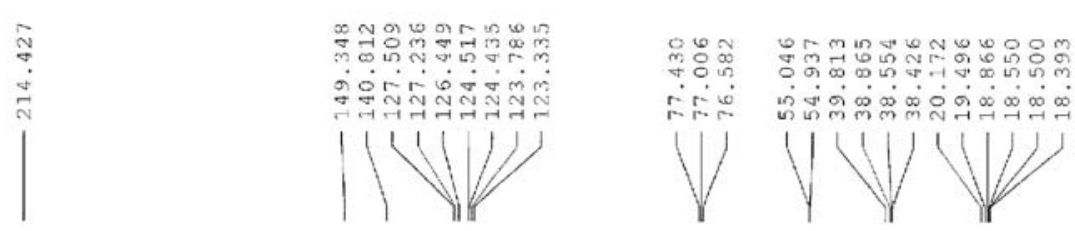<smiles>CC(C)C(=O)C1C[C@H](C)c2ccccc21</smiles>

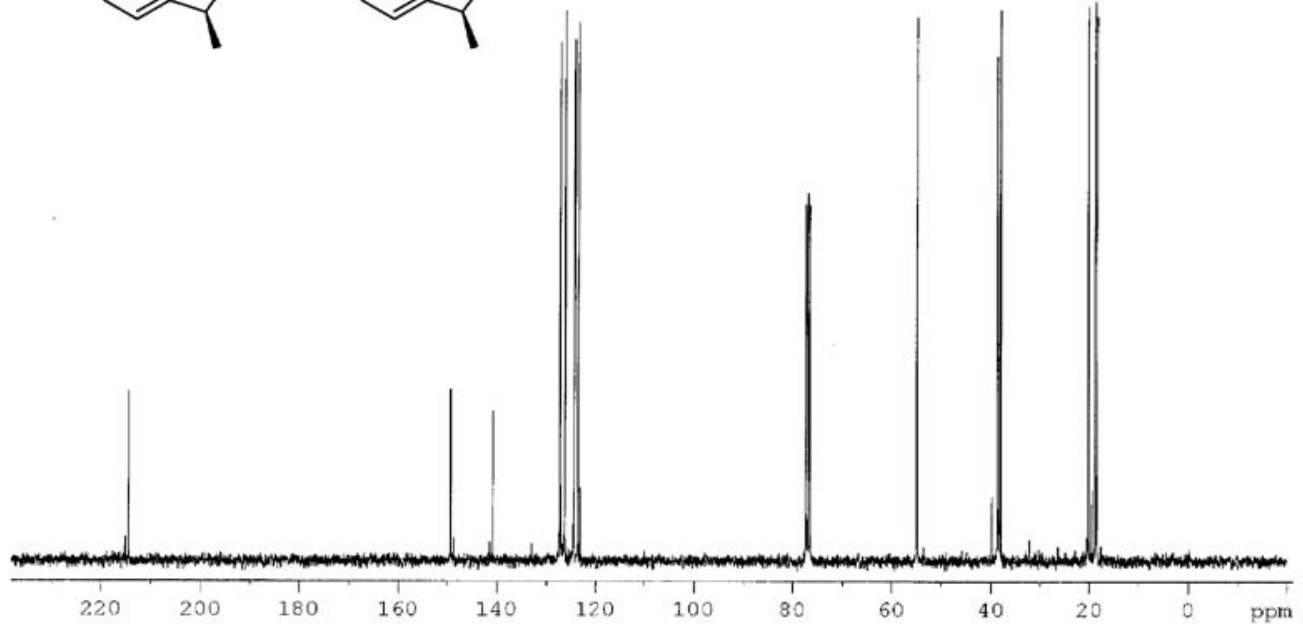

Figure S22. ${ }^{13} \mathrm{C}$ NMR spectrum of $\mathbf{5 m}\left(\mathrm{CDCl}_{3}\right.$, TMS, $\left.75 \mathrm{MHz}, \delta\right)$. 


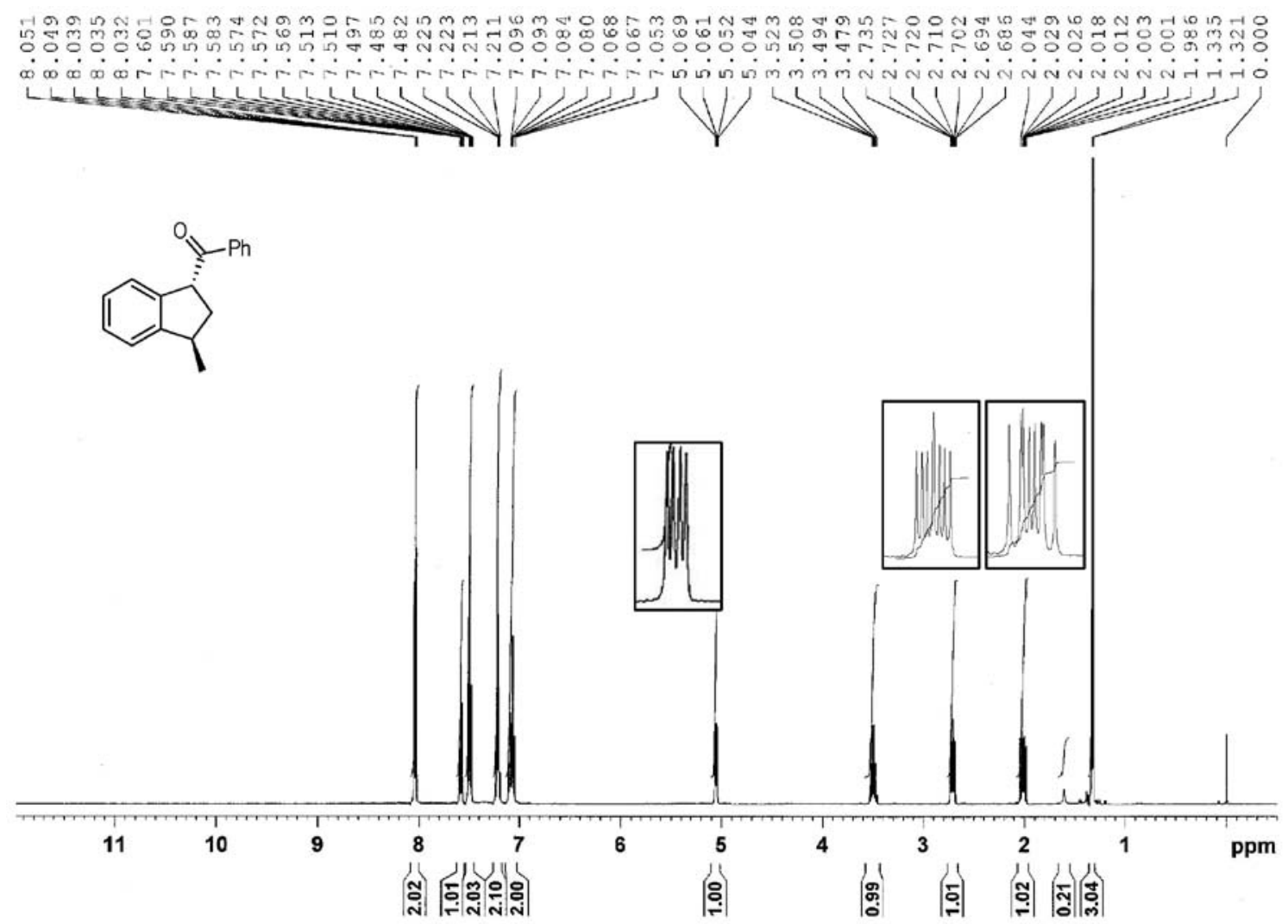

Figure S23. ${ }^{1} \mathrm{H}$ NMR spectrum of $\mathbf{5 n}\left(\mathrm{CDCl}_{3}\right.$, TMS, $\left.500 \mathrm{MHz}, \delta\right)$.

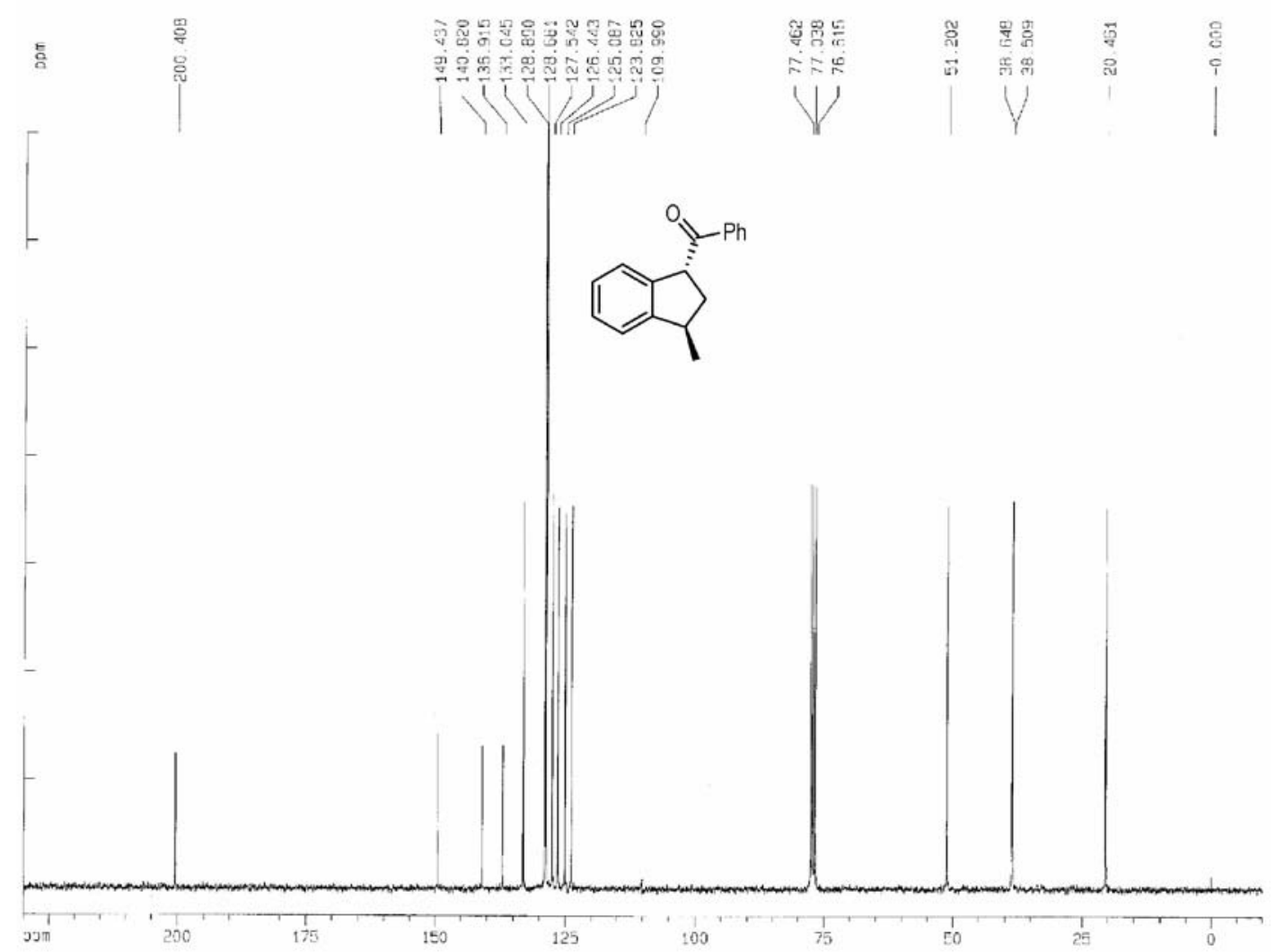

Figure S24. ${ }^{13} \mathrm{C}$ NMR spectrum of $\mathbf{5 n}\left(\mathrm{CDCl}_{3}, \mathrm{TMS}, 75 \mathrm{MHz}, \delta\right)$. 


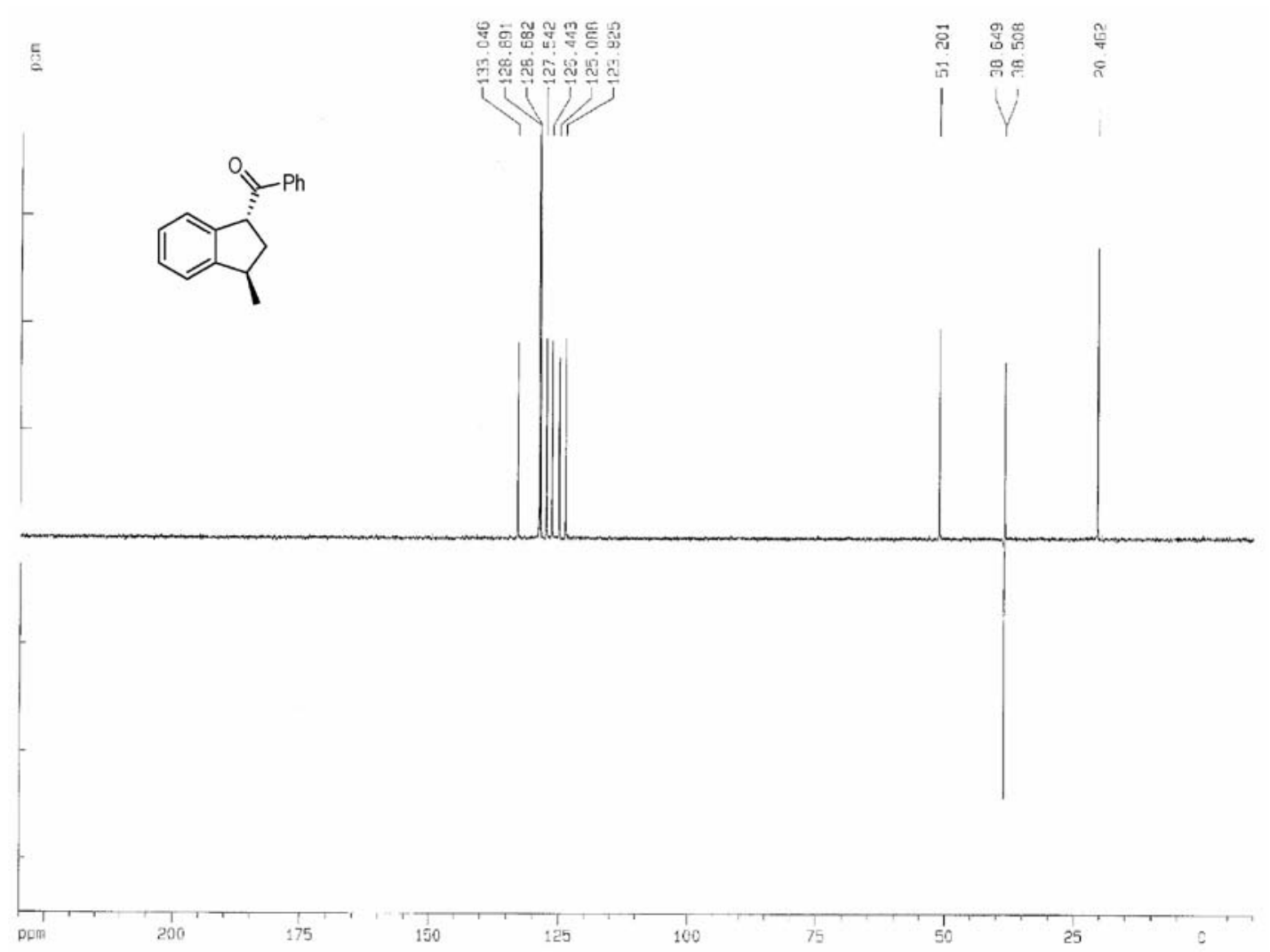

Figure S25. DEPT 135 spectrum of $\mathbf{5 n}\left(\mathrm{CDCl}_{3}, \mathrm{TMS}, 75 \mathrm{MHz}, \delta\right)$.

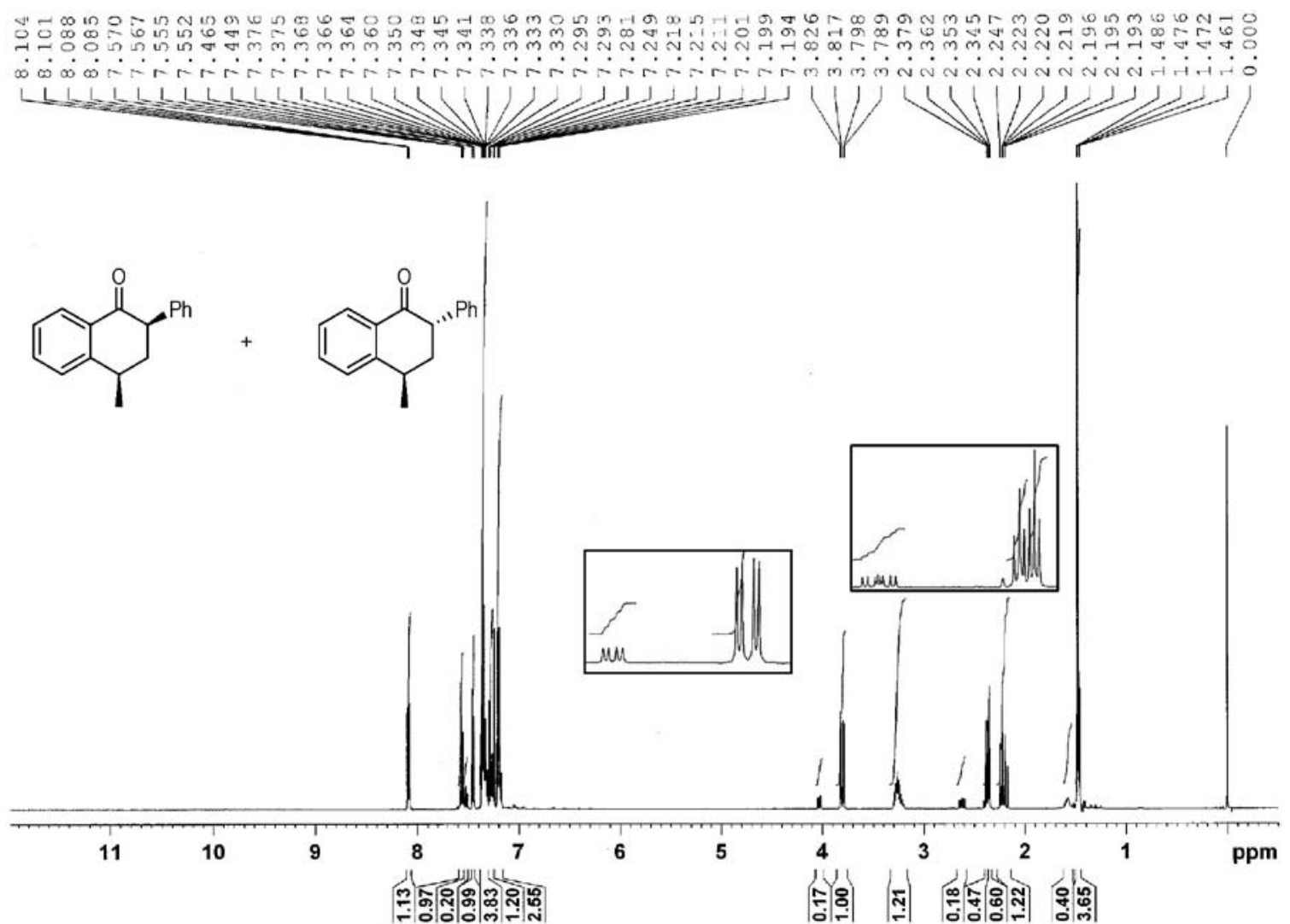

Figure S26. ${ }^{1} \mathrm{H}$ NMR spectrum of $\mathbf{6 n}\left(\mathrm{CDCl}_{3}\right.$, TMS, $\left.300 \mathrm{MHz}, \delta\right)$. 


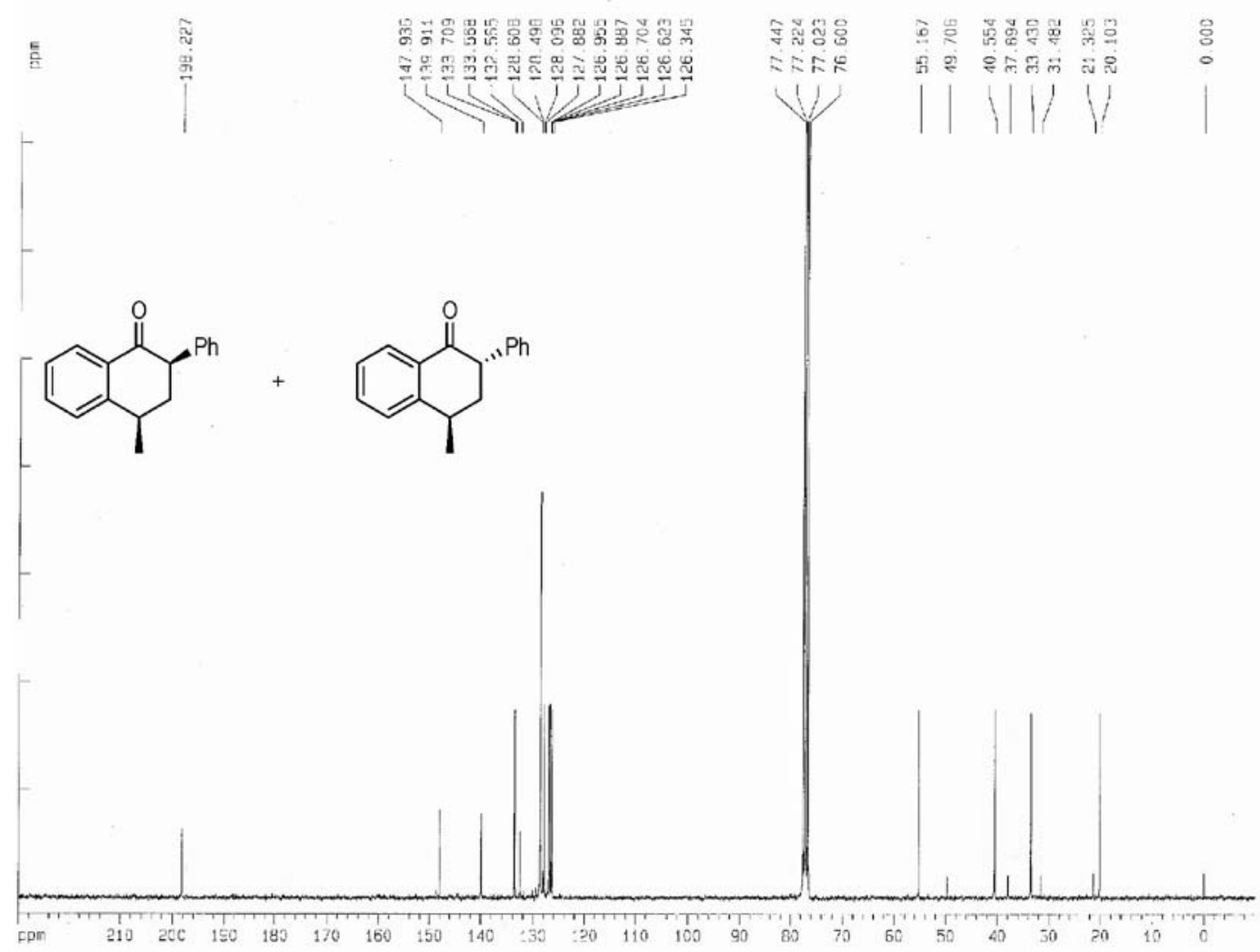

Figure S27. ${ }^{13} \mathrm{C}$ NMR spectrum of $\mathbf{6 n}\left(\mathrm{CDCl}_{3}, \mathrm{TMS}, 75 \mathrm{MHz}, \delta\right)$.

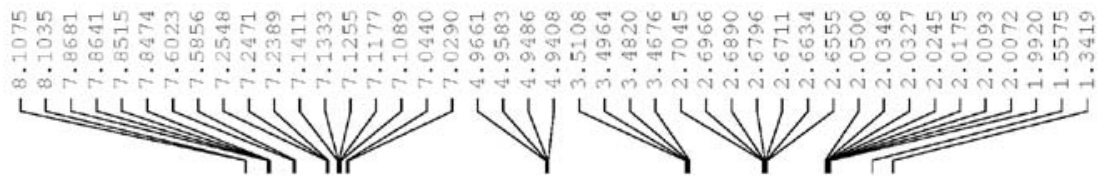<smiles>C[C@H]1C[C@H](C(=O)c2ccc(Cl)c(Cl)c2)c2ccccc21</smiles>

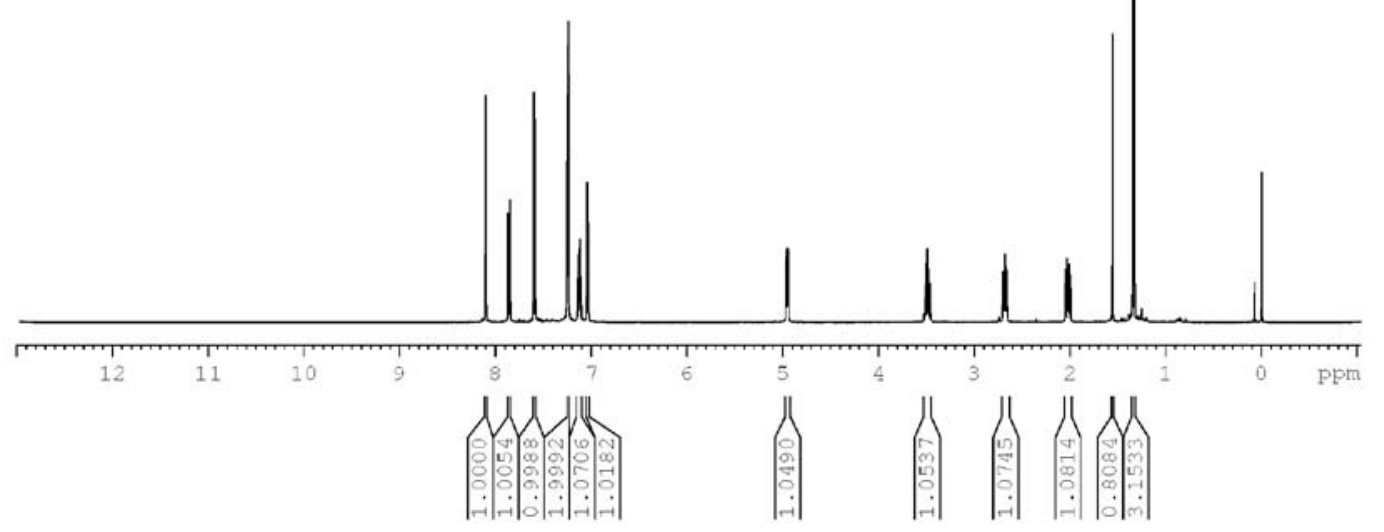

Figure S28. ${ }^{1} \mathrm{H}$ NMR spectrum of $\mathbf{5 o}\left(\mathrm{CDCl}_{3}, \mathrm{TMS}, 500 \mathrm{MHz}, \delta\right)$. 

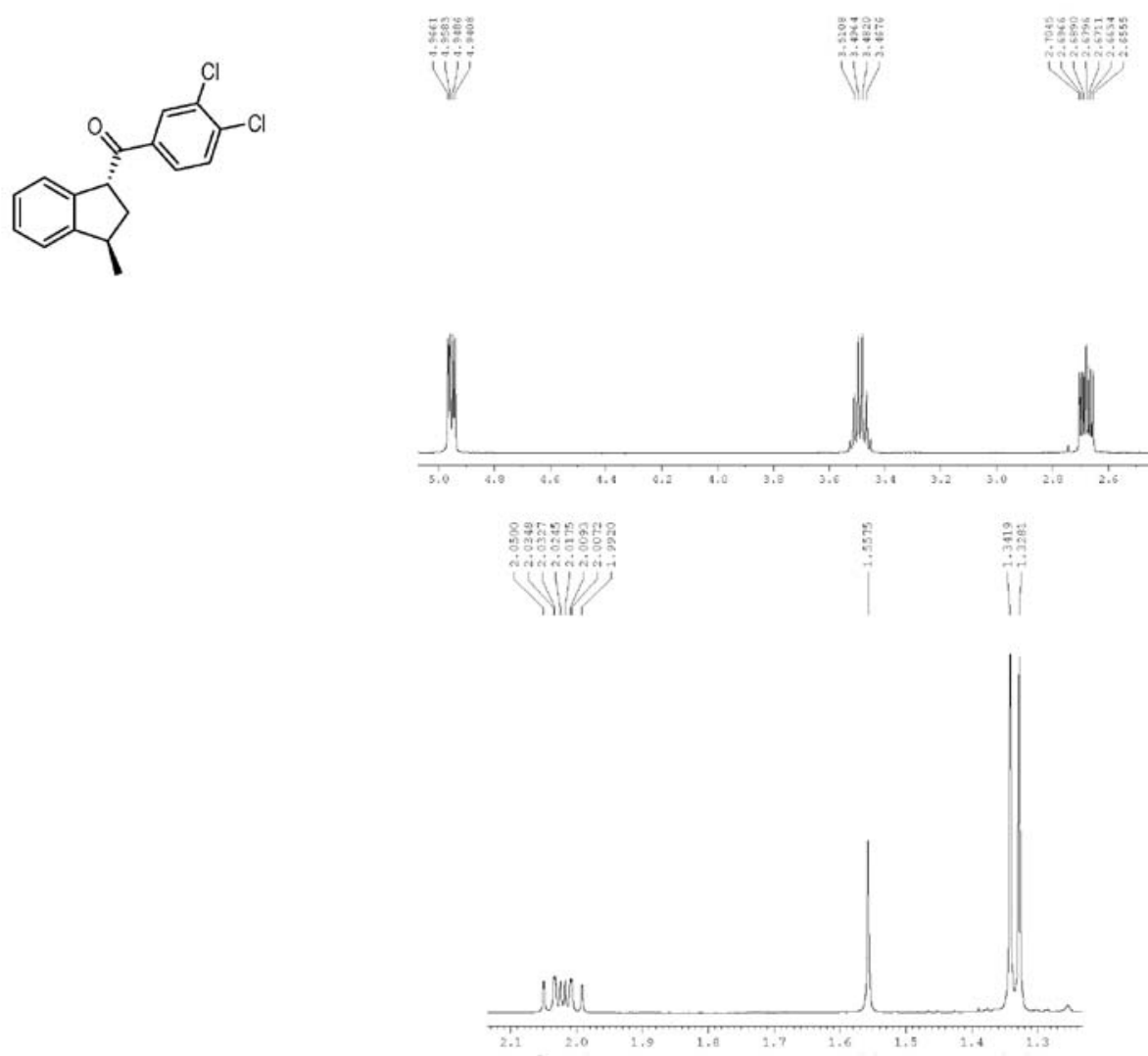

Figure S29. ${ }^{1} \mathrm{H}$ NMR spectrum of $\mathbf{5 o}\left(\mathrm{CDCl}_{3}\right.$, TMS, $\left.500 \mathrm{MHz}, \delta\right)$ - expansion.
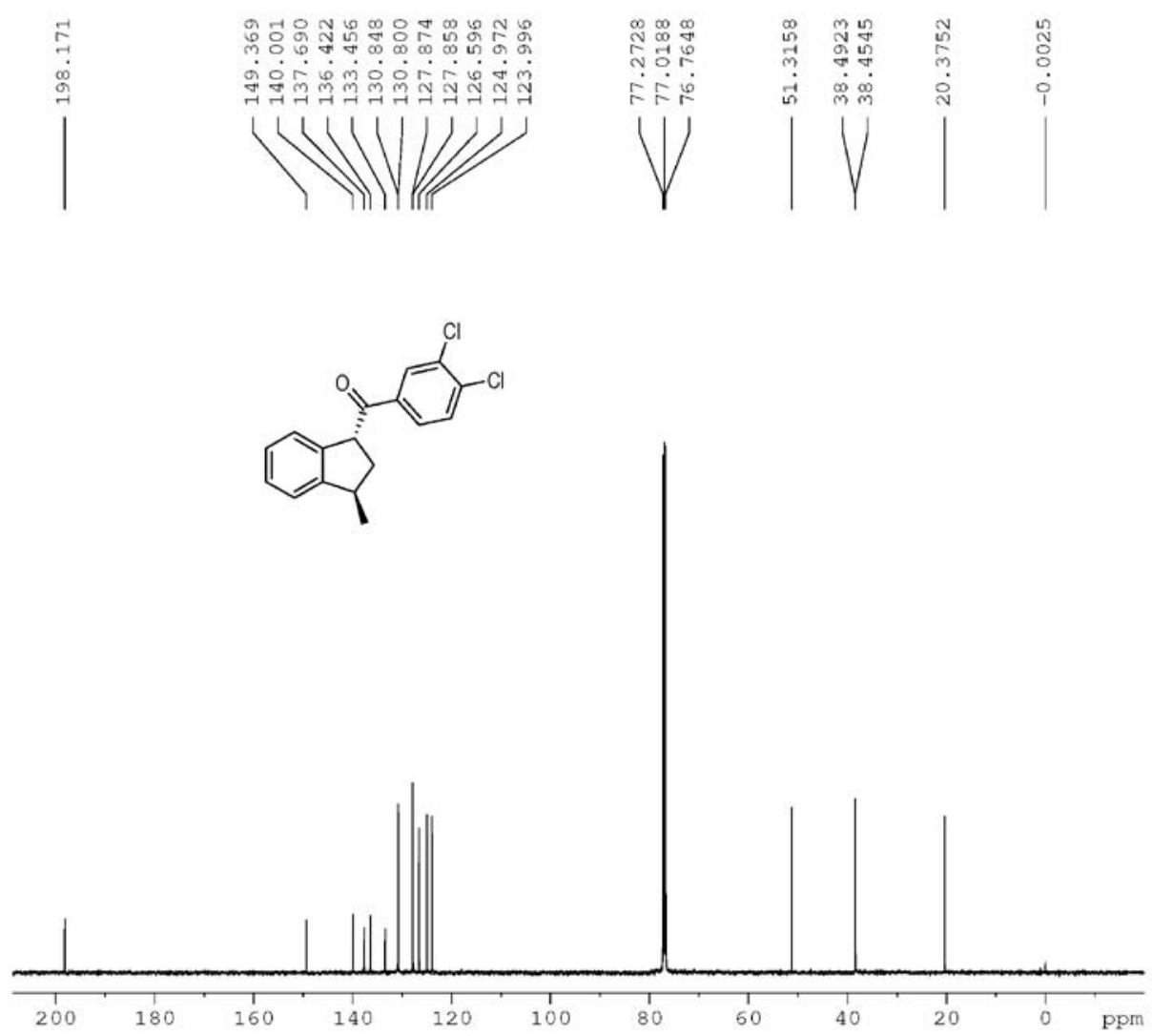

Figure S30. ${ }^{13} \mathrm{C}$ NMR spectrum of $\mathbf{5 o}\left(\mathrm{CDCl}_{3}, \mathrm{TMS}, 125 \mathrm{MHz}, \delta\right)$. 

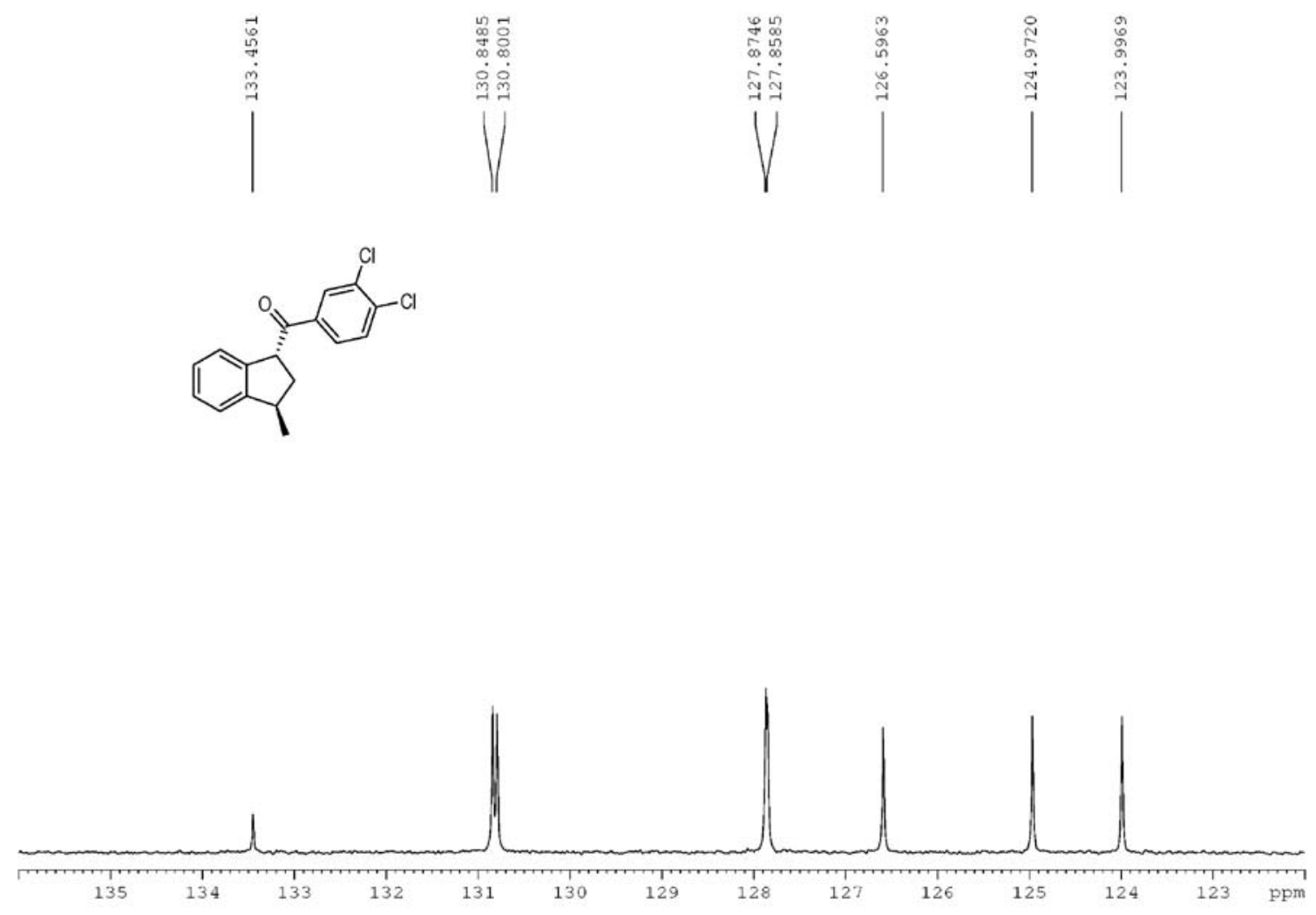

Figure S31. ${ }^{13} \mathrm{C}$ NMR spectrum of $\mathbf{5 o}\left(\mathrm{CDCl}_{3}\right.$, TMS, $\left.125 \mathrm{MHz}, \delta\right)$ - expansion.
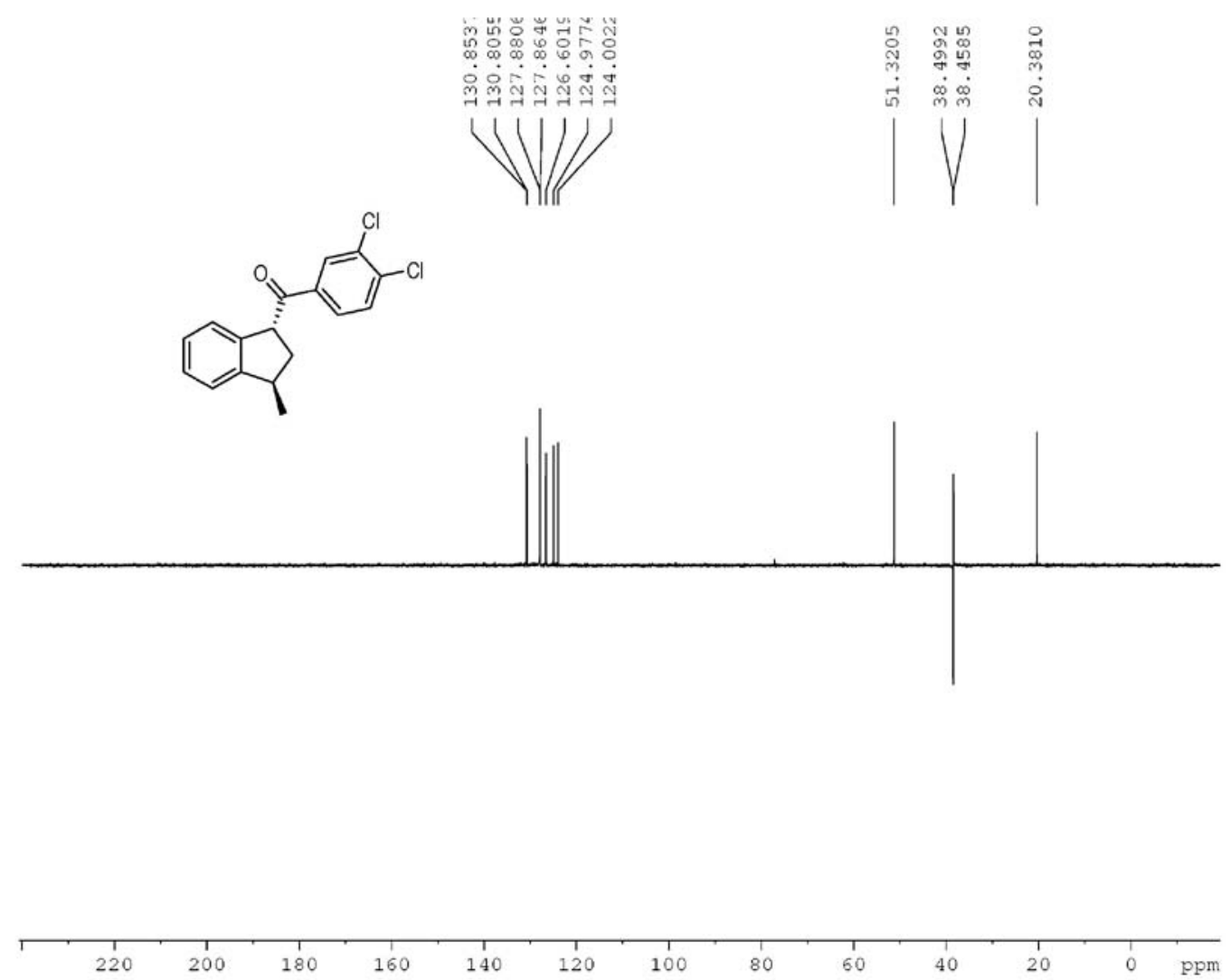

Figure S32. DEPT 135 spectrum of $\mathbf{5 o}\left(\mathrm{CDCl}_{3}, \mathrm{TMS}, 125 \mathrm{MHz}, \delta\right)$. 


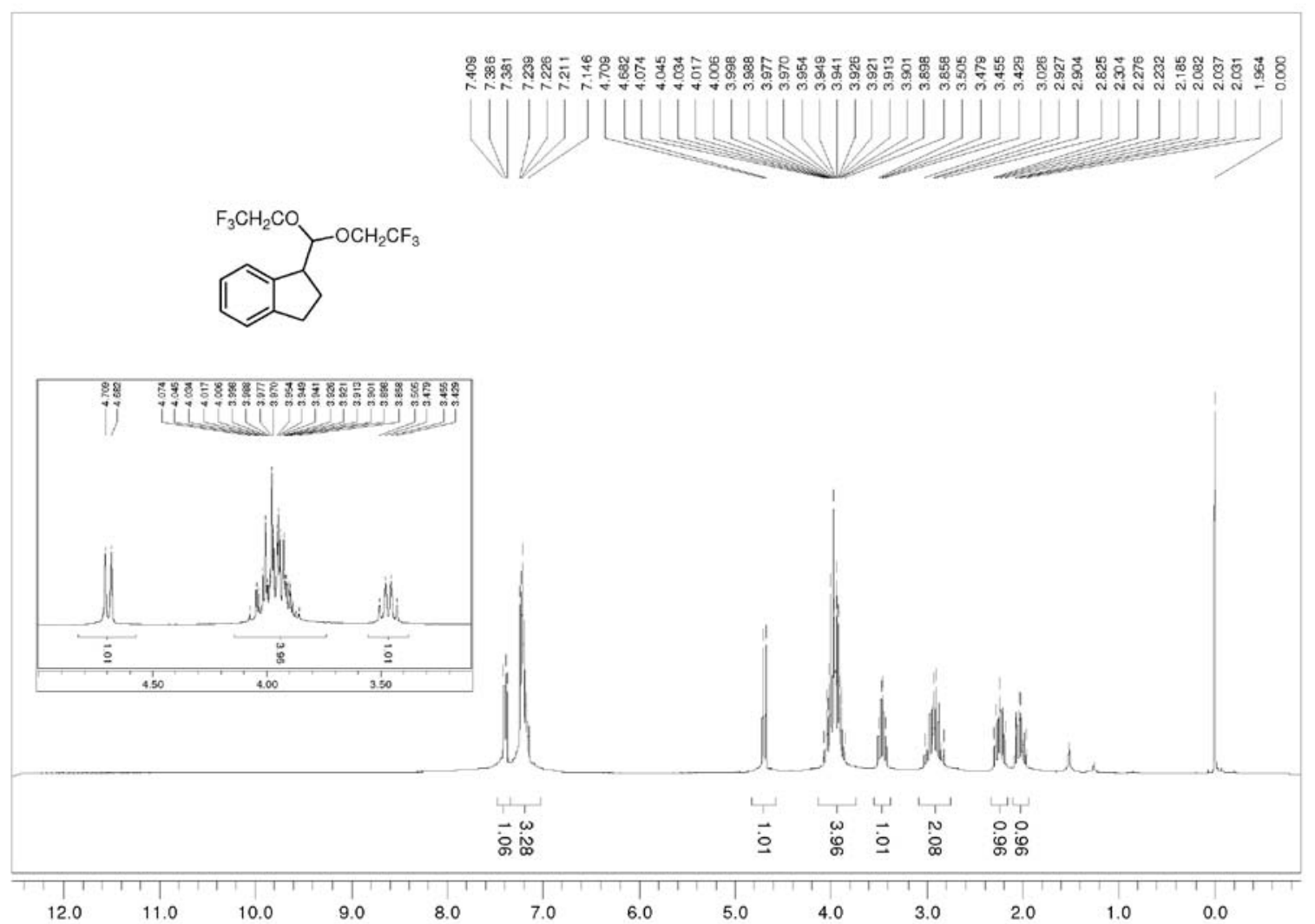

Figure S33. ${ }^{1} \mathrm{H}$ NMR spectrum of $\mathbf{8 a}\left(\mathrm{CDCl}_{3}, \mathrm{TMS}, 300 \mathrm{MHz}, \delta\right)$.

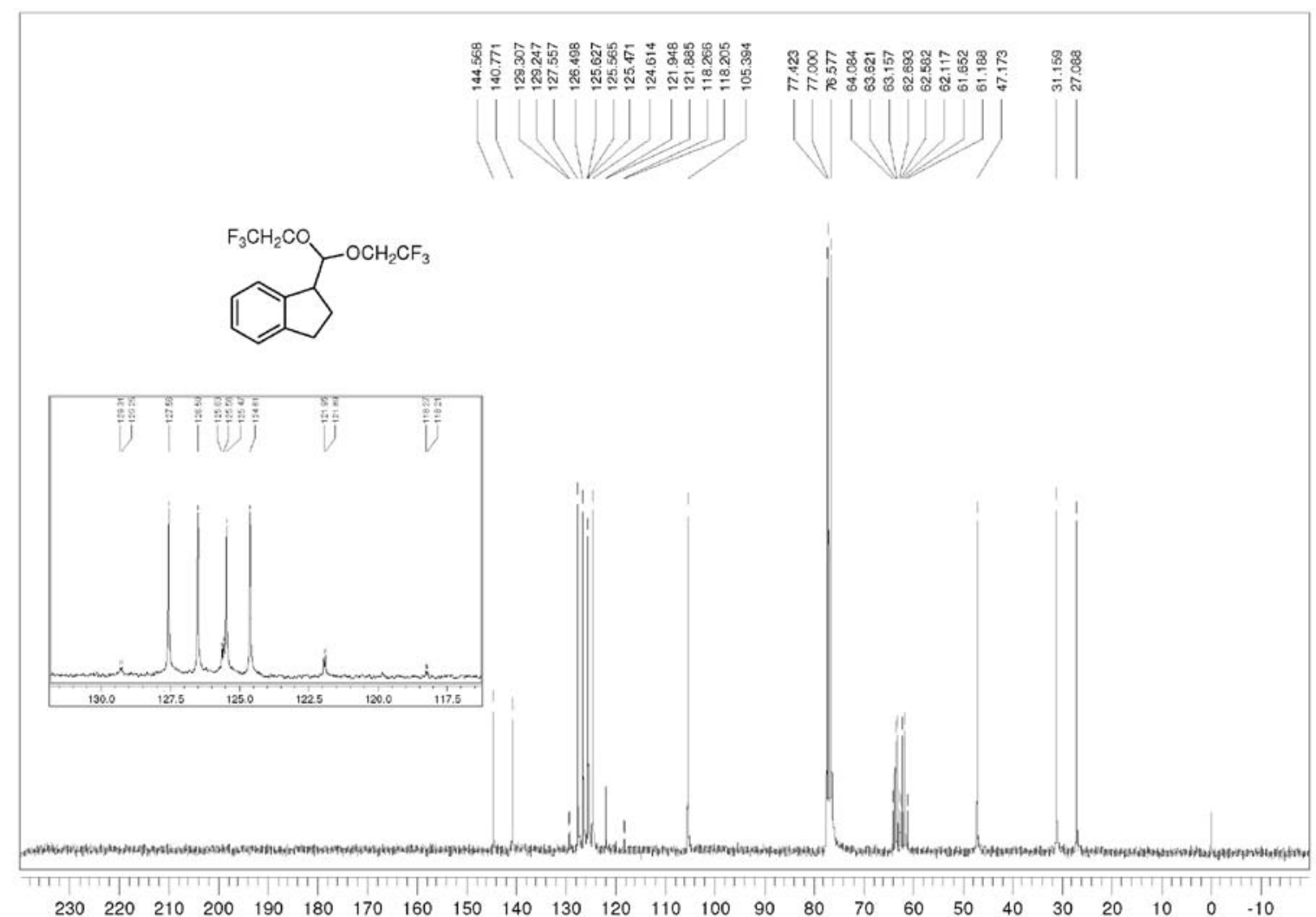

Figure S34. ${ }^{13} \mathrm{C}$ NMR spectrum of $\mathbf{8 a}\left(\mathrm{CDCl}_{3}, \mathrm{TMS}, 75 \mathrm{MHz}, \delta\right)$. 


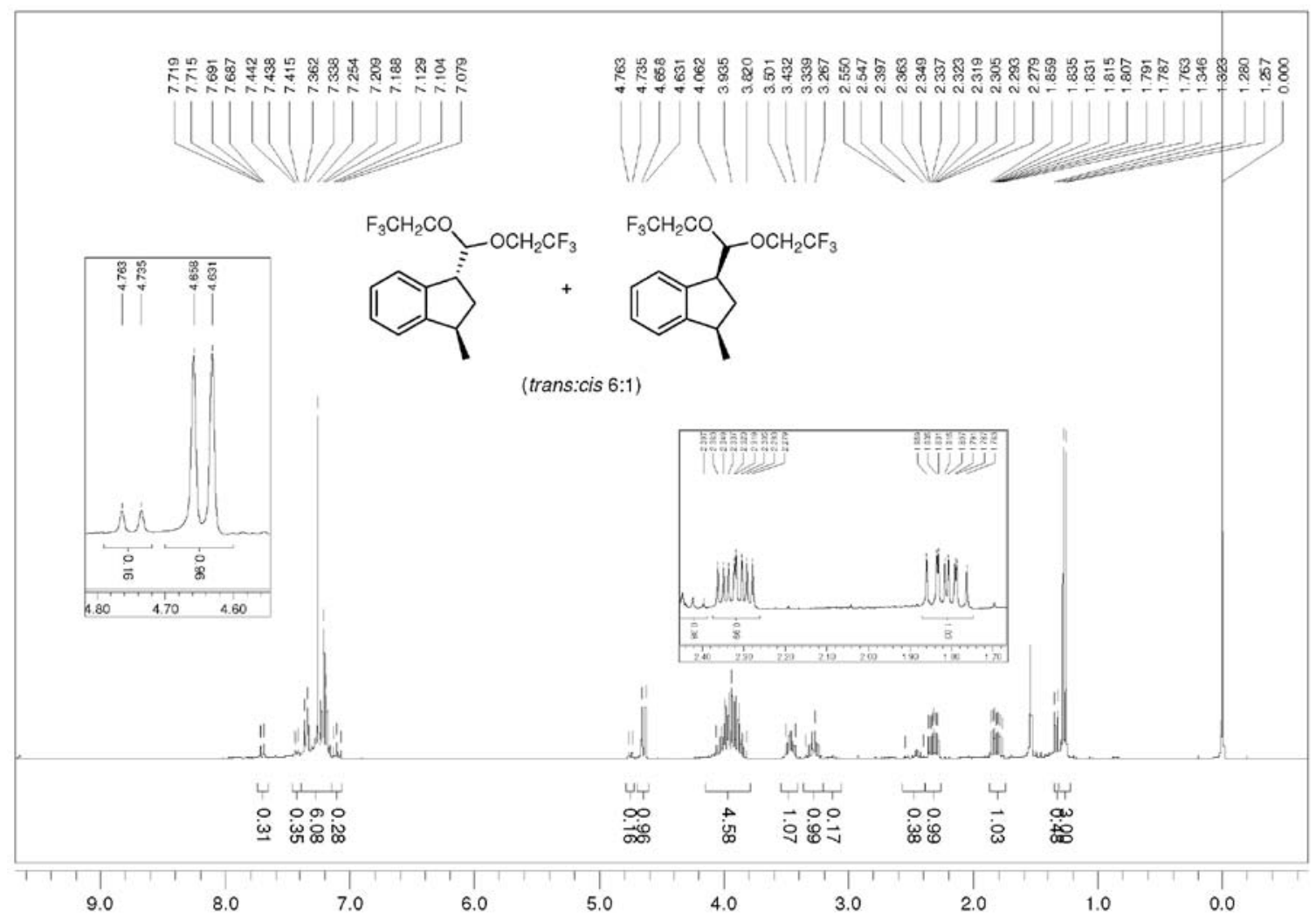

Figure S35. ${ }^{1} \mathrm{H}$ NMR spectrum of $\mathbf{8 b}\left(\mathrm{CDCl}_{3}\right.$, TMS, $\left.300 \mathrm{MHz}, \delta\right)$.

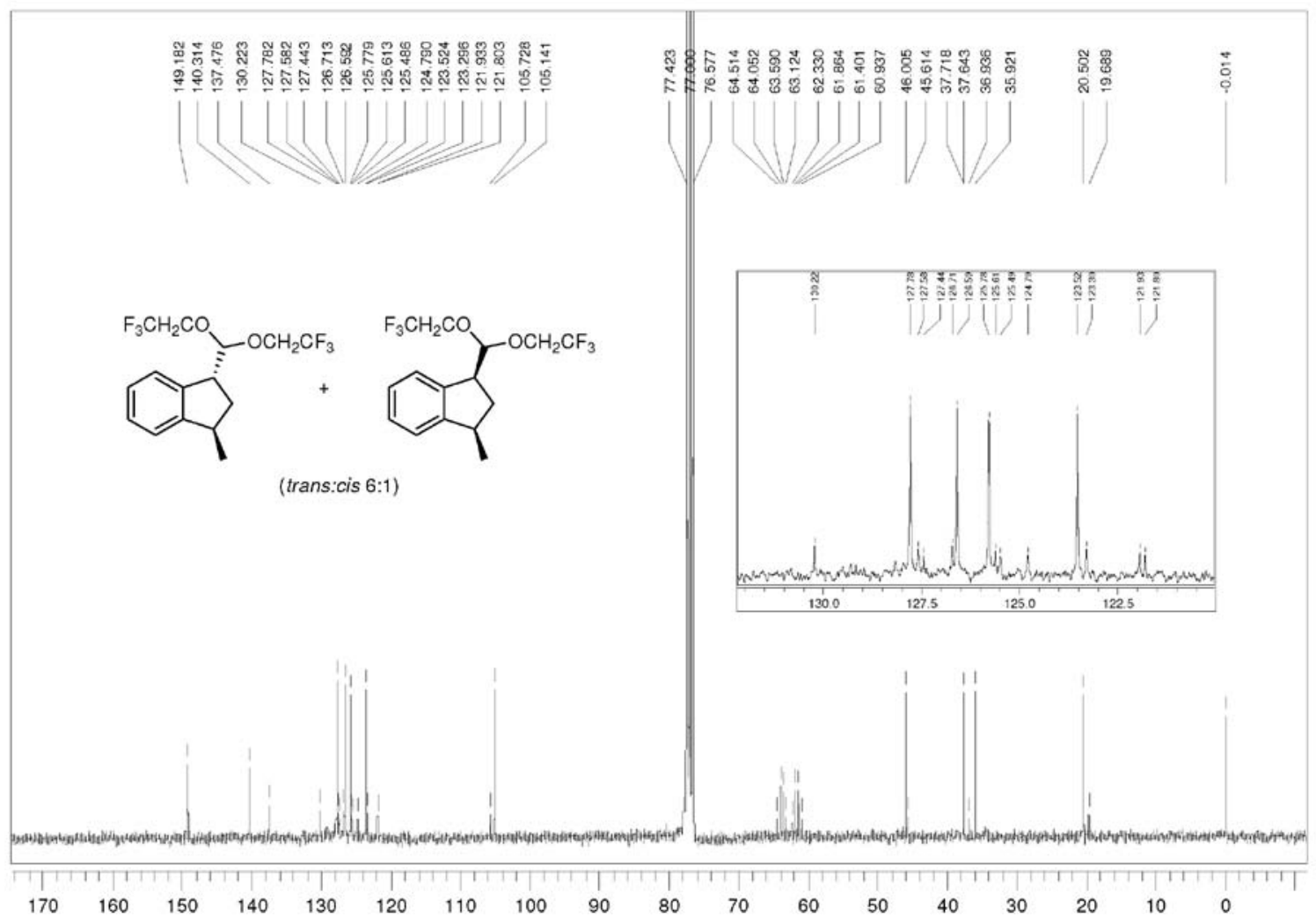

Figure S36. ${ }^{13} \mathrm{C}$ NMR spectrum of $\mathbf{8 b}\left(\mathrm{CDCl}_{3}, \mathrm{TMS}, 75 \mathrm{MHz}, \delta\right)$. 


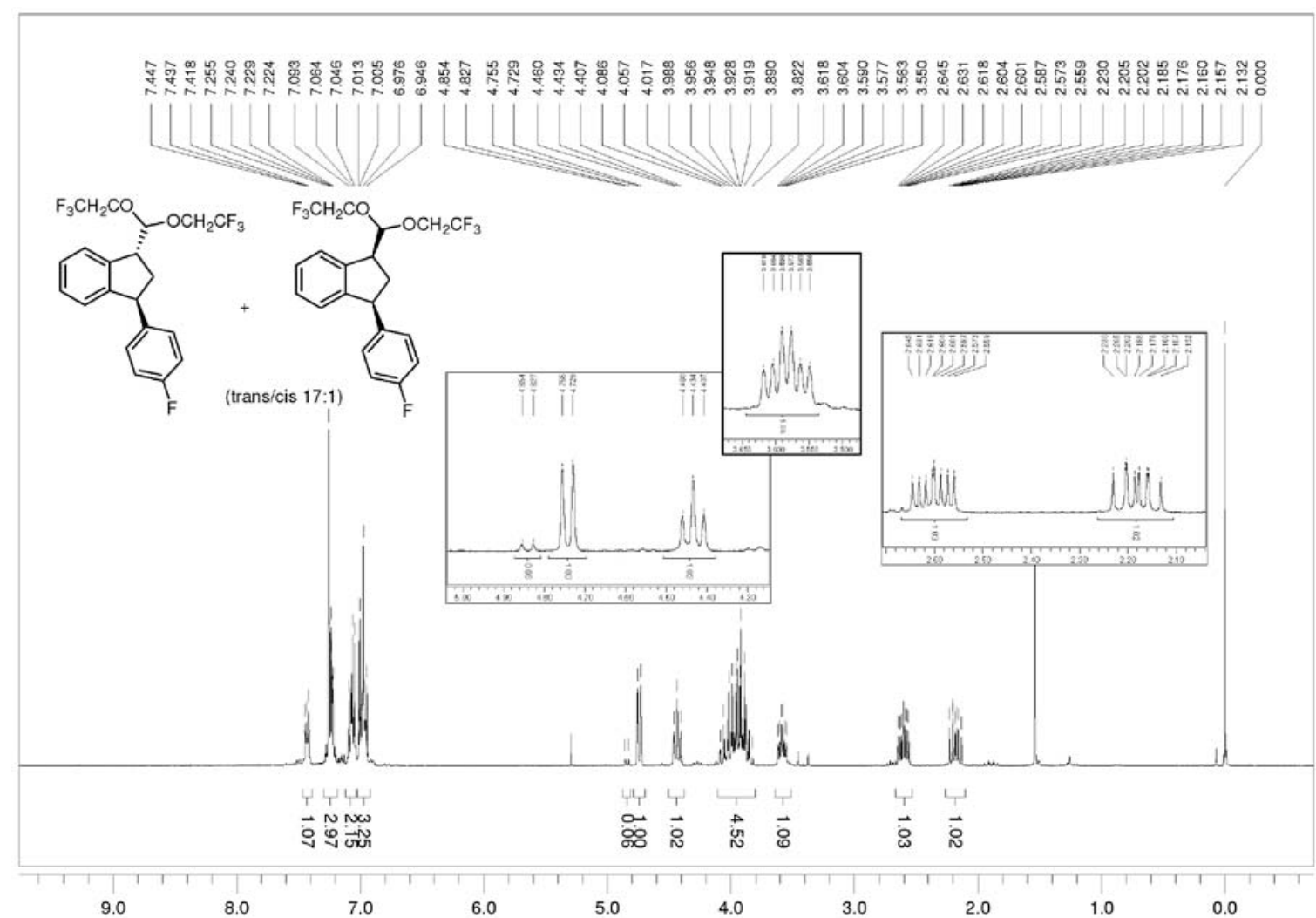

Figure S37. ${ }^{1} \mathrm{H}$ NMR spectrum of $\mathbf{8 q}\left(\mathrm{CDCl}_{3}, \mathrm{TMS}, 300 \mathrm{MHz}, \delta\right)$.

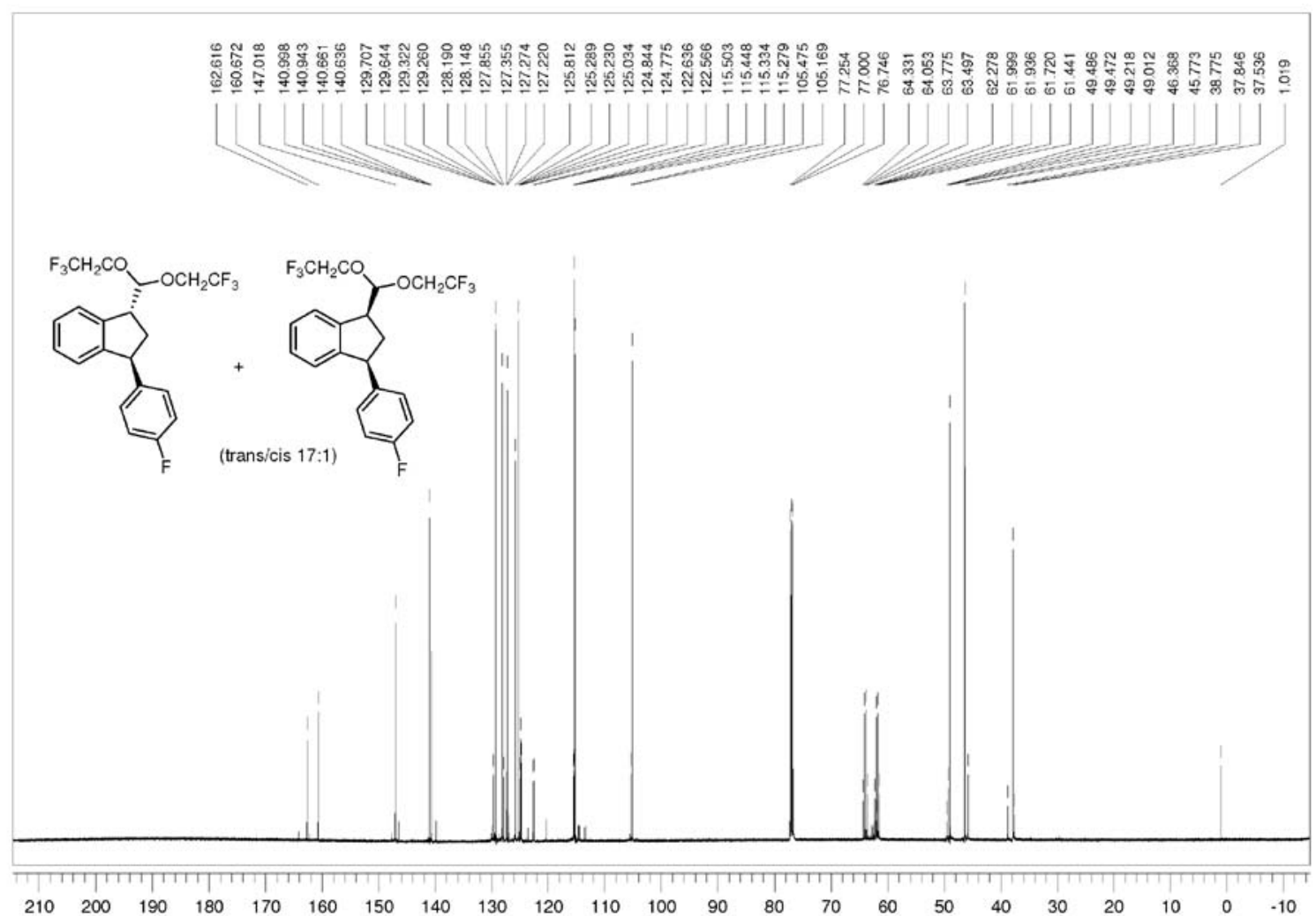

Figure S38. ${ }^{13} \mathrm{C}$ NMR spectrum of $\mathbf{8 q}\left(\mathrm{CDCl}_{3}, \mathrm{TMS}, 125 \mathrm{MHz}, \delta\right)$. 


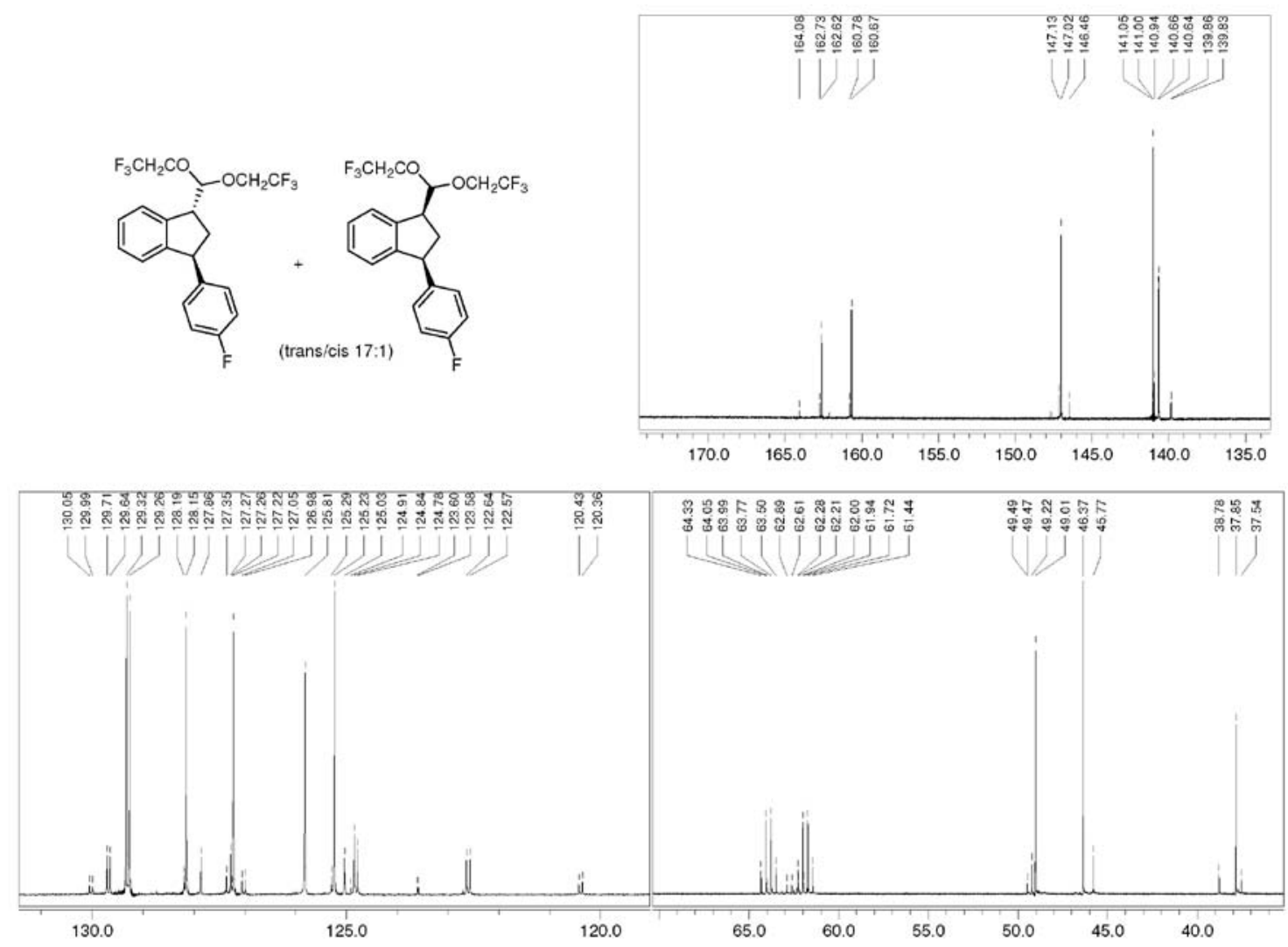

Figure S39. ${ }^{13} \mathrm{C}$ NMR spectrum of $\mathbf{8 q}\left(\mathrm{CDCl}_{3}\right.$, TMS, $\left.125 \mathrm{MHz}, \delta\right)$ - expansions.

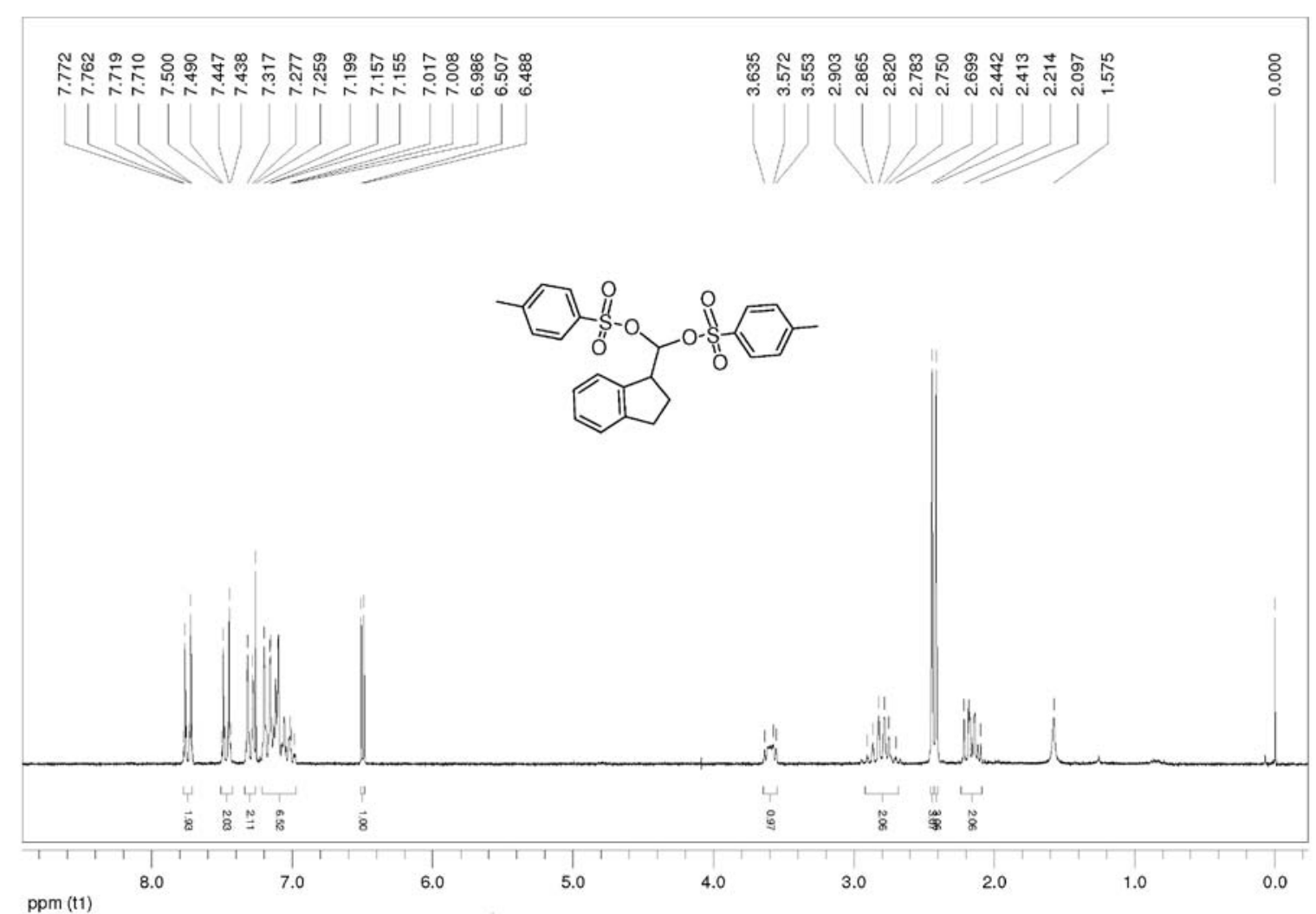

Figure S40. ${ }^{1} \mathrm{H}$ NMR spectrum of $\mathbf{1 0 a}\left(\mathrm{CDCl}_{3}, \mathrm{TMS}, 200 \mathrm{MHz}, \delta\right)$. 


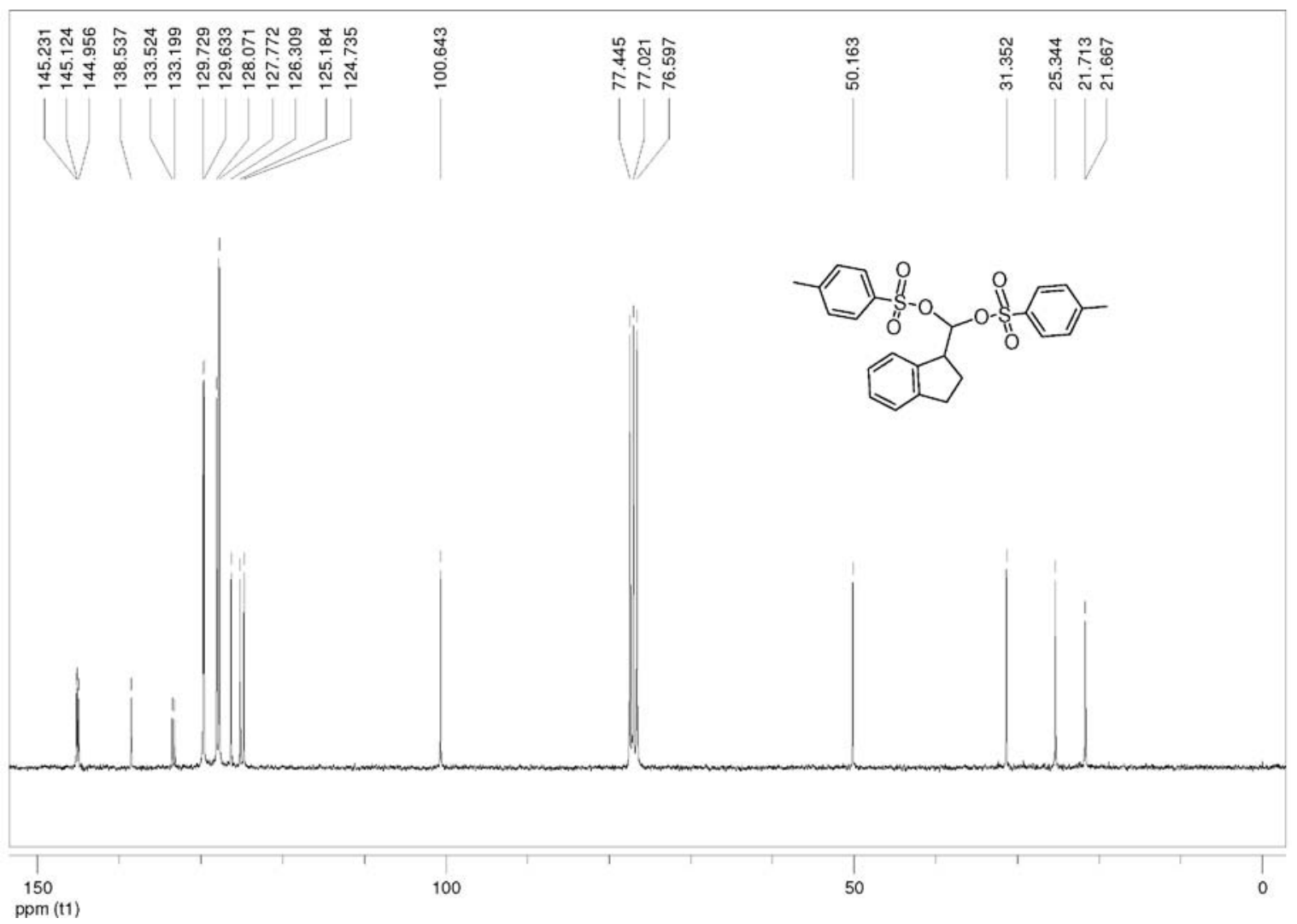

Figure S41. ${ }^{13} \mathrm{C}$ NMR spectrum of 10a $\left(\mathrm{CDCl}_{3}, \mathrm{TMS}, 50 \mathrm{MHz}, \delta\right)$.

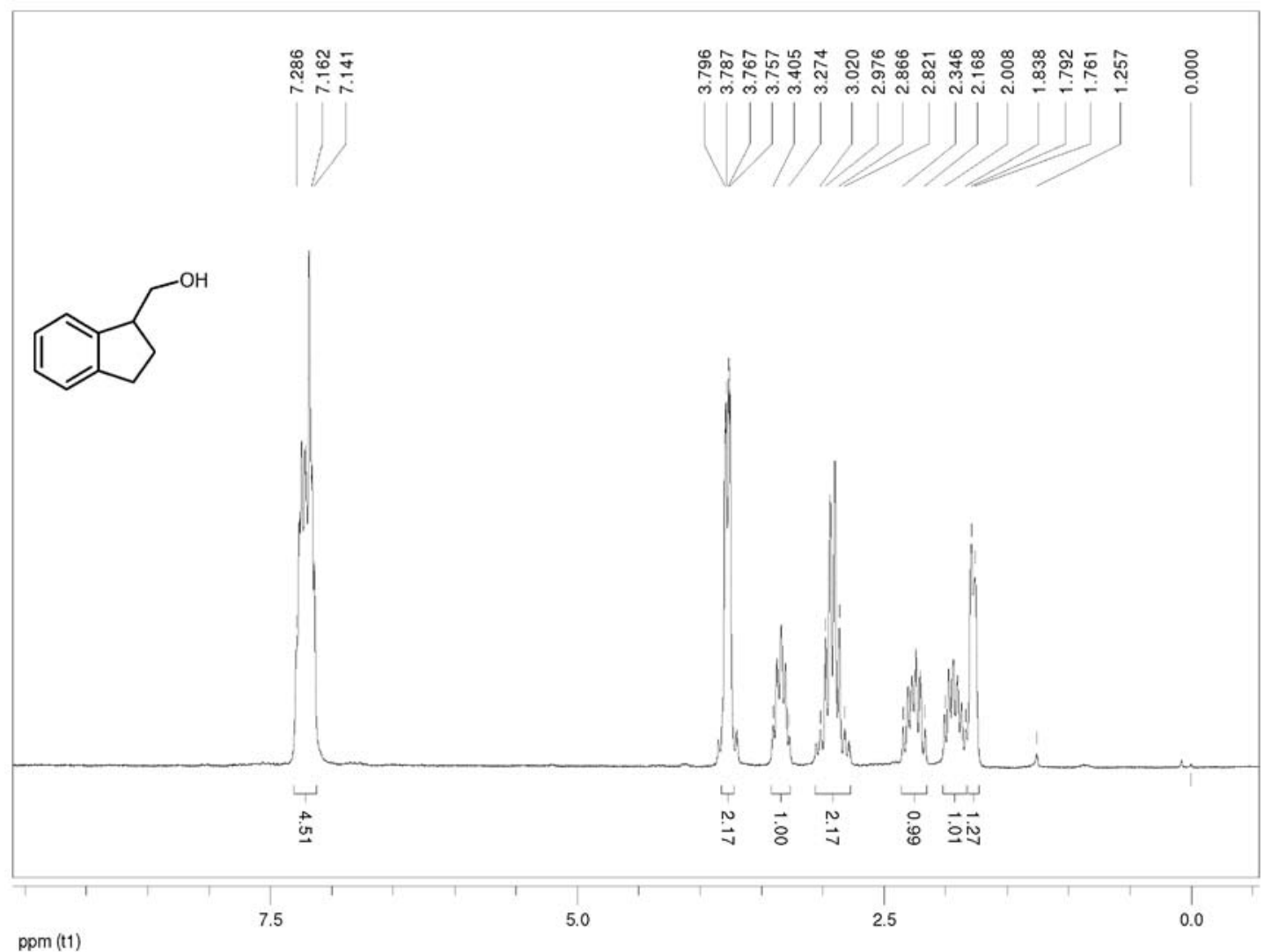

ppm (t1)

Figure S42. ${ }^{1} \mathrm{H}$ NMR spectrum of $\mathbf{9 a}\left(\mathrm{CDCl}_{3}, \mathrm{TMS}, 200 \mathrm{MHz}, \delta\right)$. 


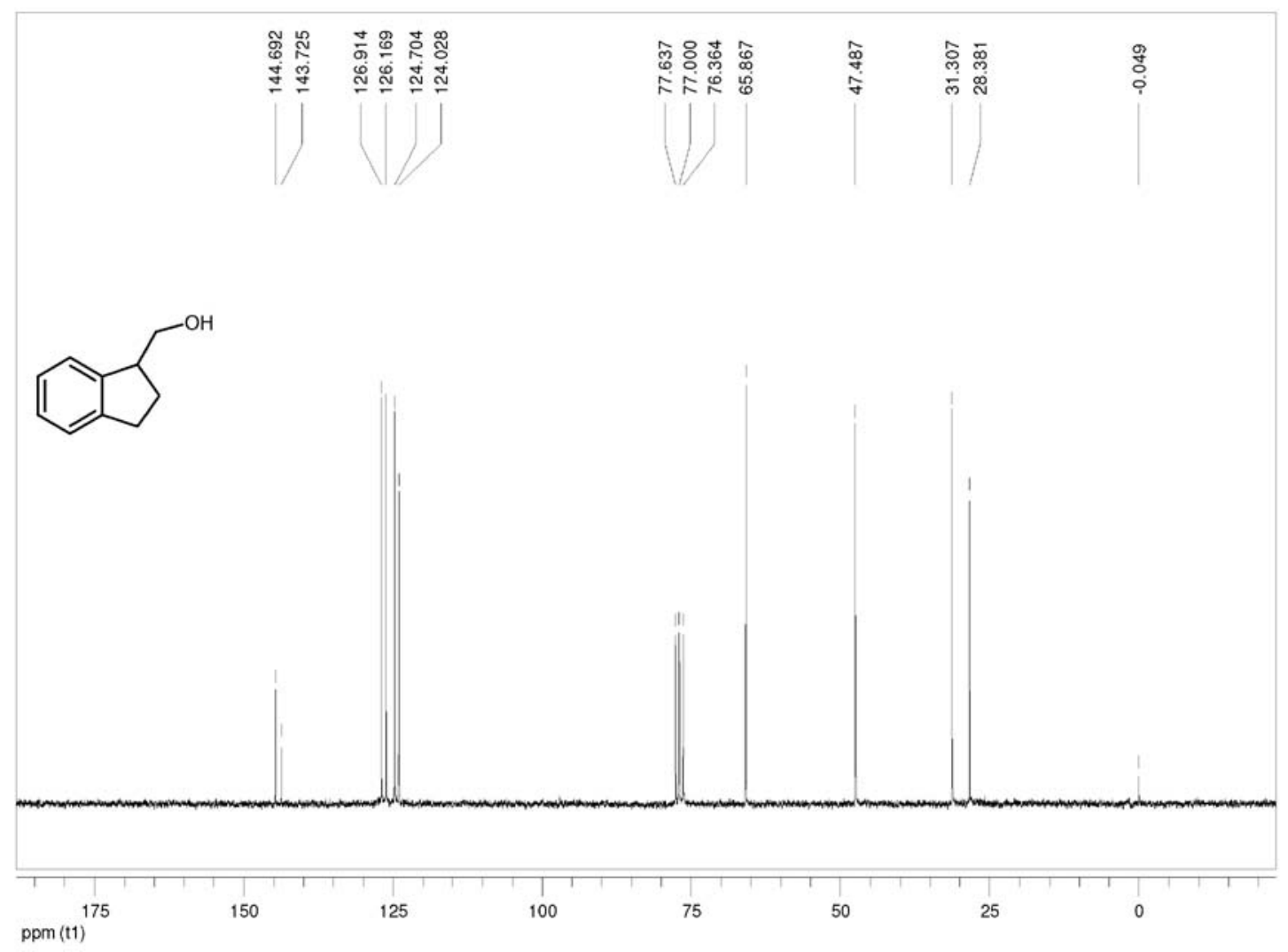

Figure S43. ${ }^{13} \mathrm{C}$ NMR spectrum of $9 a\left(\mathrm{CDCl}_{3}, \mathrm{TMS}, 50 \mathrm{MHz}, \delta\right)$. 